\title{
2 Non-canonical regulation of glycogenolysis and the Warburg 3 phenotype by soluble adenylyl cyclase
}

\section{Authors}

6

7 Jung-Chin Chang ${ }^{1,2}$, Simei Go ${ }^{1,2}$, Eduardo H. Gilglioni ${ }^{1,3}$, Hang Lam Li ${ }^{1,4}$, Hsu-Li Huang ${ }^{1,4}$,

8 Lonny R. Levin ${ }^{5}$, Jochen Buck ${ }^{5}$, Arthur J. Verhoeven ${ }^{1}$, Ronald P.J. Oude Elferink ${ }^{1,2}$

9

\section{Affiliations}

11

12

${ }^{1}$ Tytgat Institute for Liver and Intestinal Research, Amsterdam UMC, University of Amsterdam,

13 Amsterdam, the Netherlands

$14{ }^{2}$ Amsterdam Gastroenterology and Metabolism (AG\&M) Research Institute, Amsterdam UMC,

15 University of Amsterdam, Amsterdam, the Netherlands

$16{ }^{3}$ University of Maringá, Department of Biochemistry, Paraná, Brazil

$17{ }^{4}$ School of Medicine, National Taiwan University, Taipei, Taiwan

$18{ }^{5}$ Department of Pharmacology, Weill Cornell Medical College, New York, USA 


\section{Abstract (169/175)}

21

22 Cyclic AMP is produced in cells by two very different types of adenylyl cyclases: the canonical 23 transmembrane adenylyl cyclases (tmACs, $A D C Y 1 \sim 9$ ) and the evolutionarily more conserved soluble 24 adenylyl cyclase (SAC, $A D C Y 10$ ). While the role and regulation of tmACs is well documented, much 25 less is known of SAC in cellular metabolism. We demonstrate here that SAC is an acute regulator of 26 glycolysis, oxidative phosphorylation and glycogen metabolism, tuning their relative bioenergetic 27 contributions. Suppression of SAC activity leads to aerobic glycolysis, enhanced glycogenolysis, 28 decreased oxidative phosphorylation, and an elevated cytosolic NADH/NAD ratio, resembling the 29 Warburg phenotype. Importantly, we found that glycogen metabolism is regulated in opposite 30 directions by cAMP depending on its location of synthesis and downstream effectors. While the 31 canonical tmAC-cAMP-PKA axis promotes glycogenolysis, we identify a novel sAC-cAMP-Epac1 axis 32 that suppresses glycogenolysis. These data suggest that SAC is an autonomous bioenergetic sensor 33 that suppresses aerobic glycolysis and glycogenolysis when ATP levels suffice. When the ATP level 34 falls, diminished SAC activity induces glycogenolysis and aerobic glycolysis to maintain energy 35 homeostasis. 


\section{Introduction}

Since the discovery of $3^{\prime}, 5^{\prime}$-cyclic adenosine monophosphate (cAMP) by Earl Sutherland in his mechanistic study of glycogen metabolism (Sutherland \& Rall, 1958), cAMP generated by

41 transmembrane adenylyl cyclases (tmACs, ADCY1 ADCY9) has been recognized as a versatile second messenger mediating many downstream signals of G-protein-coupled receptors (GPCRs). Recently, a new type of mammalian soluble adenylyl cyclase ( $S A C, A D C Y 10$ ) was discovered, which is evolutionarily more conserved than tmACs and is localized not at the plasma membrane but intracellularly. (Chen, Cann et al., 2000, Kamenetsky, Middelhaufe et al., 2006). The intracellularly localized SAC and the plasma-membrane-localized tmACs share common cAMP effectors, such as protein kinase A (PKA) and "exchange factor directly activated by cAMP" (Epac1 and Epac2). However, SAC is not regulated by G-proteins and does not respond to forskolin (Kamenetsky et al., 2006). Instead, SAC activity is positively regulated by physiological concentrations of bicarbonate and finetuned by $\mathrm{Ca}^{2+}$ (Chen et al., 2000, Kleinboelting, Diaz et al., 2014, Litvin, Kamenetsky et al., 2003).

While the tmACs, regulated by $\mathrm{G}$ protein-coupled receptors in response to hormones and neurotransmitters, evolved subsequent to multicellularity, the evolutionary more ancient sAC-like enzymes first appeared in unicellular organisms and might regulate autonomous functions of cells. This concept is supported by the conservation of bicarbonate-mediated activation of sAC homologs in multicellular as well as in unicellular organisms (Kobayashi, Buck et al., 2004). As bicarbonate and $\mathrm{CO}_{2}$ are the primary product of substrate oxidation by the TCA cycle, we hypothesized that SAC regulates the autonomous metabolism of cells and this is supported by several characteristics of the enzyme. Firstly, sAC can directly sense the cellular energetic state due to its high $K_{\mathrm{m}}$ for its substrate ATP (ranging from 1 to $10 \mathrm{mM}$ ) (Jaiswal \& Conti, 2003, Litvin et al., 2003, Zippin, Chen et al., 2013). Secondly, albeit at low level, SAC is expressed in almost all tissues examined (Geng, Wang et al., 2005, Levin \& Buck, 2015). Thirdly, $\mathrm{Ca}^{2+}$, another versatile and universal second messenger, stimulates sAC in synergy with bicarbonate (Geng et al., 2005, Jaiswal \& Conti, 2003), allowing sAC to tune the cellular metabolism according to ongoing cellular signaling. In line with this reasoning, the ATP- and calcium-sensing properties of SAC have been demonstrated to facilitate diverse metabolic regulations, including the regulation of oxidative phosphorylation by sensing $\mathrm{CO}_{2}$ production from the TCA cycle (Acin-Perez, Salazar et al., 2009) and/or free $\mathrm{Ca}^{2+}$ concentrations in the mitochondrial matrix (Di Benedetto, Scalzotto et al., 2013), induction of ATP-dependent insulin secretion in the pancreas (Zippin et al., 2013), secretion of aldosterone in adrenal cortex carcinoma H295R (Katona, 
Rajki et al., 2015), and TNF-induced respiratory burst in human neutrophils (Han, Stessin et al., 2005).

Over the past decade, it has become widely accepted that cAMP signaling is compartmentalized into microdomains which allows this single messenger molecule to mediate disparate functions even within a single cell. (Kamenetsky et al., 2006, Lefkimmiatis \& Zaccolo, 2014). We have therefore examined the role of SAC-derived CAMP in energy metabolism and found that SAC acts as an acute switch for aerobic glycolysis and glycogenolysis in maintaining autonomous energy metabolism of cells. In multiple tested cell lines, acute suppression of SAC induces aerobic glycolysis, increased glycogenolysis and increased cytosolic $\mathrm{NADH} / \mathrm{NAD}^{+}$ratios. Apart from the established control of oxidative phosphorylation by SAC through regulation of complex I activity, we identified a novel regulatory mechanism of SAC on glycogen metabolism that is opposite to the well-established pathway of glycogenolysis by tmACs. Thus, while the tmAC-PKA signaling axis promotes glycogenolysis, a novel SAC-Epac1 signaling axis inhibits glycogen breakdown.

\section{Results}

\section{Suppression of soluble adenylyl cyclase stably reprograms cell metabolism towards} aerobic glycolysis

To investigate the role of SAC in autonomous metabolism of cells, we examined whether SAC activity regulates glycolysis at steady state. As SAC protein expression is generally low, we first used the SV-40 large T antigen immortalized human intrahepatic cholangiocyte cell line H69 (hereafter H69 cholangiocytes) (Grubman, Perrone et al., 1994), whose high endogenous expression of sAC was previously validated pharmacologically and genetically (Chang, Go et al., 2016). Consistent with previous studies, two SAC-specific inhibitors KH7 and LRE1 (Ramos-Espiritu, Kleinboelting et al., 2016b) suppressed cAMP production in H69 cholangiocytes (Figure S1A and S1B). Addition of KH7 or LRE1 elicited a dose-dependent increase in glucose consumption in $\mathrm{H} 69$ cholangiocytes. The increased glucose consumption was accompanied by increased lactate secretion and reduced pyruvate secretion, which resulted in an elevation of the lactate-to-pyruvate ratio in the medium in cells treated with sAC inhibitors (Figure 1A and 1B). The increased lactate-to-pyruvate ratio in media mirrored a similar change in intracellular lactate-to-pyruvate ratio (Fig. S2A). Similar changes in glucose, lactate and pyruvate were observed in SAC-knockdown H69 cholangiocytes (Figure 1C and 
101 1D). Next, we investigated the general aspect of this observation with the human hepatoma cell line

102 HepG2 (Fig. 1E and S2B) as well as with primary mouse hepatocytes and three additional cell lines

103 of different tissue origin (human colorectal adenocarcinoma Caco-2, Abelson mouse leukemia virus-

104 transformed macrophage RAW264.7, and mouse melanoma cell line B16F10; Figure S1C-F).

105 Inhibition of SAC increased lactate secretion, and reduced pyruvate secretion in all four cell lines as

106 well as primary mouse hepatocytes. Although the baseline lactate-to-pyruvate ratios were different

107 in each cell type, SAC inhibition always led to an elevated medium lactate-to-pyruvate ratio. These

108 findings show that both pharmacological and genetic suppression of SAC activity promotes aerobic

109 glycolysis under normoxic conditions, resembling the Warburg effect observed in many cancer cells

110 and consistent with the previously observed tumor suppression effect of SAC (Ramos-Espiritu, Diaz

111 et al., 2016a).

113 Soluble adenylyl cyclase regulates the cytosolic NADH/NAD ${ }^{+}$redox state and aerobic 114 glycolysis via complex I

115 The monocarboxylate transporters (MCTs) on the plasma membrane equilibrates the extracellular 116 lactate-to-pyruvate ratio with the intracellular lactate-to-pyruvate ratio (Halestrap \& Wilson, 2012),

117 which is in near equilibrium with the cytosolic redox couple NADH/NAD via lactate dehydrogenase 118 (LDH) (Figure 2A). Since SAC inhibition increased the lactate-to-pyruvate ratio in both media (Figure 119 1) and inside cells (Figure S2A and S2B), we tested if SAC indeed regulates the cytosolic NADH/NAD ${ }^{+}$ 120 redox state. To this end, we expressed the Peredox NADH/NAD+ biosensor (Hung, Albeck et al., 2011) 121 in HepG2 cells to detect the ratio of free NADH over free NAD in the cytosol. The Peredox biosensor 122 responded to various ratios, but not quantities, of lactate and pyruvate in the medium, confirming 123 that the medium lactate-to-pyruvate ratio is in equilibrium with the cytosolic NADH/NAD ratio 124 (Figure 2B). We found that the addition of LRE1 acutely and stably increased cytosolic NADH/NAD 125 ratio in the presence of glucose (Figure 2C). As expected, when cells were acutely starved (Figure 2D) 126 or when they were fueled with the short chain fatty acid octanoate alone, which supports oxidative 127 phosphorylation in the absence of glycolysis (Figure $2 \mathrm{E}$ ), the baseline cytosolic NADH/NAD ${ }^{+}$ratio was 128 lower than in the presence of glucose. Interestingly, under these two conditions without 129 extracellular glucose, SAC inhibition strongly increased the NADH/NAD ${ }^{+}$ratio in a transient fashion, 130 indicating a rapid generation and subsequent depletion of glycolytic intermediates. These sAC131 dependent changes in $\mathrm{NADH} / \mathrm{NAD}^{+}$ratio are dependent on glycolytic flux. When acutely starved cells 132 were treated with 2-deoxyglucose to block glycolysis, the cytosolic NADH/NAD ratio reduced 133 dramatically and the effect of the SAC inhibitor LRE1 was abolished (Figure 2F). Similarly, inhibiting 
134 glyceraldehyde-3-phosphate dehydrogenase (GAPDH), the NADH-generation step in glycolysis, with

135 iodoacetate also significantly lowered cytosolic NADH/NAD ${ }^{+}$ratio and blocked the effect of sAC

136 inhibition (Figure 2G). These data demonstrate that cytosolic NADH is maintained by glycolysis and

137 that SAC inhibition promotes glycolytic flux to increase the cytosolic NADH/NAD ${ }^{+}$ratio, which

138 becomes transient in the absence of glucose.

We next tested if SAC inhibition increased the cytosolic NADH/NAD ${ }^{+}$ratio by decreasing NADH oxidation in mitochondria. While SAC has been reported to regulate various components of the mitochondrial respiratory chain in isolated mitochondria, the implicated target has not been consistent between the various reports (Acin-Perez et al., 2009, De Rasmo, Micelli et al., 2016, De Rasmo, Signorile et al., 2015). We reasoned that this might be due to differences in isolation procedures and the consequential disruption of mitochondrial networks. To minimize the disruption of the mitochondrial network and local structures, we examined the ADP-driven (state 3 ) respiration and FCCP-driven respiration (with ATP synthase blocked by oligomycin A) in digitonin-permeabilized HepG2 cells instead of isolated mitochondria. When mitochondria were fueled with complex I substrate (pyruvate), SAC inhibition significantly suppressed both ADP-driven and FCCP-driven respiration (Figure $2 \mathrm{H}-2 \mathrm{~J}$ and S2C-S2E for pyruvate/malate and glutamate/malate, respectively). However, when mitochondria were fueled with complex II substrate (succinate), sAC inhibition only very mildly suppressed ADP-driven respiration and had no effect on FCCP-driven respiration (Figure $2 \mathrm{~K}-2 \mathrm{M})$. Thus, inhibiting SAC diminishes oxygen consumption primarily in the context of complex I driven respiration. In line with this, inhibiting complex I-dependent OCR with rotenone mimicked the Warburg-like phenotype observed in the SAC-suppressed state: increased lactate secretion, decreased pyruvate secretion, and elevated medium lactate-to-pyruvate ratio (Figure S2F-S2H).

157 These results suggest that $\mathrm{SAC}$ regulates the cytosolic NADH/NAD ${ }^{+}$redox state and aerobic glycolysis 158 by affecting complex I activity. homeostasis permeabilized cells, we asked if SAC can be an acute switch for aerobic glycolysis. To this end, we monitored glycolysis and oxidative phosphorylation by simultaneously measuring the extracellular acidification rate (ECAR) and oxygen consumption (OCR) in a Seahorse Flux Analyzer. As this method 
examined whether endogenous bicarbonate production was sufficient to maintain SAC activity within the time frame of our experiments. We found that acute removal of $\mathrm{CO}_{2}$ in the gas phase did not affect SAC activity, suggesting SAC is primarily activated by endogenously produced bicarbonate

170 (Figure S3A). In the presence of glucose, inhibiting SAC by LRE1 increased the extracellular 171 acidification rate (ECAR) and decreased the coupled oxygen consumption rate (OCR) in both H69 172 (Figure 3A and 3B) and HepG2 cells (Figure 3C and 3D). Importantly, these metabolic effects of sAC 173 inhibition took place within minutes and were stable. FCCP-uncoupled respiration was also 174 suppressed upon SAC inhibition. To investigate whether energy homeostasis was maintained upon 175 inhibition of SAC activity, we derived the ATP production rates of glycolysis and mitochondria from extracellular flux measurements in intact HepG2 cells following the method described by Mookerjee 177 et al. (Mookerjee, Gerencser et al., 2017, Mookerjee, Goncalves et al., 2015). We found that in the 178 presence of glucose, SAC inhibition acutely lowered ATP production by both oxidative 179 phosphorylation and TCA cycle activity while increasing ATP production by glycolysis (Figure S3BS3E). The overall ATP production remained unchanged (Figure 3SF). Direct determination of adenylate nucleotides in the cytosol also showed no changes in adenylate energy charge (Figure 3SG). These findings demonstrate that in the presence of glucose SAC maintains ATP homeostasis by reciprocally regulating ATP production via glycolysis and oxidative phosphorylation.

We next examined how SAC regulates glycolysis and oxidative phosphorylation in the absence of glucose. When HepG2 cells were acutely starved (Figure 3E and 3F) or fueled solely with octanoate

187 (Figure $3 \mathrm{G}$ and $3 \mathrm{H}$ ), a membrane-permeant short-chain fatty acid and a direct substrate for 188 mitochondrial $\beta$-oxidation, the effects of SAC inhibition on ECAR and coupled OCR remained reciprocal but became only transient. Calculation of the concomitant ATP production showed that ATP production by glycolysis was transiently stimulated upon SAC inhibition while ATP production by TCA cycle and oxidative phosphorylation were transiently suppressed (Figure S3H-S3K). Once again, as in the presence of glucose, the overall ATP production remained constant, resulting in an unchanged adenylate energy charge in the cytosol (Figure S3L and S3M). However, while SAC inhibition suppressed FCCP-uncoupled respiration in the presence of glucose, sAC inhibition did not affect FCCP-uncoupled respiration when cells were starved of glucose or fueled with octanoate. Thus, glucose is required for the stable inhibition of oxidative phosphorylation when SAC is inhibited under both coupled and uncoupled conditions. Indeed, when glucose was added to octanoate-fueled HepG2 cells, the effects of SAC inhibition on ECAR and OCR were stabilized (Figure $3 \mathrm{I}$ and $3 \mathrm{~J}$ ). Moreover, glucose also restored LRE1-induced suppression of FCCP-uncoupled respiration in octanoate-fueled HepG2 cells. Conversely, when HepG2 cells were fueled with octanoate in the 
presence of 2-deoxyglucose, the transient increase of ECAR was completely blocked and the degree of suppression of coupled OCR was reduced (Figure 3K and 3L). These data show that sAC inhibition suppresses oxidative phosphorylation by enhancing glycolysis in both the presence and absence of glucose, but in the absence of exogenously supplied glucose these changes are transient. The transient nature of these changes in the absence of glucose suggests that glycolytic intermediates may be responsible for the regulation of oxidative phosphorylation.

\section{Inhibition of soluble adenylyl cyclase promotes glycogenolysis}

209 In the absence of exogenous glucose, ECAR was transiently stimulated by sAC inhibition, which 210 implies that SAC inhibition increases availability of a glycolytic fuel. We hypothesized that these 211 transient changes reflected the mobilization of a cellular glycolytic reserve, namely glycogen. While 212 liver and muscles are the primary organs for glycogen storage, glycogen can be detected in tumor 213 cell lines of various tissue origins (Rousset, Zweibaum et al., 1981). Indeed, we found that both 214 HepG2 and H69 cells store significant quantities of glycogen (Figure 4A and 4B). In both cell lines, inhibition of SAC reduced glycogen stores within one hour. In HepG2 cells, sAC inhibition greatly reduced glycogen content in the absence of glucose but only mildly affected it in the presence of

217 glucose (Figure 4A). In H69, sAC inhibition caused net glycogen breakdown both in the presence and 218 absence of extracellular glucose (Figure 4B). Similarly, SAC inhibition promoted glycogen breakdown in acutely starved primary mouse hepatocytes, which could be prevented by inhibiting glycogen phosphorylase $a$ with CP-91149 (Figure S4B). Furthermore, SAC inhibition also reduced glycogen content of primary mouse hepatocytes in the presence of glucose (Figure S4C). The induction of

222 glycogenolysis in $\mathrm{H} 69$ human cholangiocytes and primary mouse hepatocytes upon SAC inhibition in 223 the presence of glucose demonstrates that, at least in certain cell types, sAC-regulated glycogen 224 turnover even plays a role in energy metabolism under normoglycemic conditions.

We next examined whether glycogenolysis was directly responsible for the transient changes in 227 ECAR, OCR, and cytosolic NADH/NAD ratio observed in the absence of extracellular glucose. In 228 HepG2 cells fueled with only octanoate, the glycogen phosphorylase $a$ inhibitor CP-91149 blocked the transient increase of ECAR evoked by SAC inhibition and significantly relieved suppression of OCR, 230 showing that the transient increase of ECAR and suppression of OCR was the consequence of 231 glycogenolysis (Figure 4C-4D). Similarly, in HepG2 cells acutely starved of substrates, CP-91149 also 232 blocked the transient increase of ECAR by SAC inhibition, alleviated the transient OCR suppression, and preserved glycogen content (Figure $4 \mathrm{E}-4 \mathrm{~F}$ and $\mathrm{S} 4 \mathrm{~A}$ ). This confirms again that in the absence of 
exogenous glucose, LRE1-induced glycogenolysis fuels glycolysis, which in turn suppresses oxidative phosphorylation.

To test whether glycogenolysis-derived glycolysis was responsible for the transient increase in 238 cytosolic NADH/NAD ${ }^{+}$ratio upon SAC inhibition, we depleted glycogen by glucose starvation for 4 239 hours. In glycogen-depleted cells, sAC inhibition no longer increased the cytosolic NADH/NAD ${ }^{+}$ratio 240 (Figure 4G-4H, S4D). Thus, in the absence of glucose, sAC inhibition promotes glycogenolysis which 241 increases glycolytic flux and the cytosolic NADH/NAD ${ }^{+}$ratio.

Since $S A C$ regulates complex I activity, we examined whether the induction of glycogenolysis upon SAC inhibition was merely the metabolic consequence of suppressed complex I activity or the result of parallel signaling events. To this end, we examined whether the complex I inhibitor rotenone could phenocopy the metabolic effect of sAC inhibition. In glucose-fed HepG2 cells, rotenone at 10-15 nM suppressed both coupled and uncoupled OCR to a comparable extent as $50 \mu \mathrm{M}$ LRE1 (Figure S4E-G). However, rotenone only marginally induced glycogen breakdown and lactate secretion while LRE1 significantly depleted cellular glycogen and induced lactate secretion in octanoate-fed HepG2 cells (Figure $4 \mathrm{I}-\mathrm{J}$ ). Consistently, rotenone did not significantly increase ECAR in octanoate-fed HepG2 cells (Figure 4K). These data strongly suggest that SAC regulates glycogenolysis independently of its effect on complex I.

SAC-CAMP-Epac1 and tmAC-CAMP-PKA form two distinct microdomains that signal opposite effects on glycogenolysis

The versatility of cAMP has led to the concept of signaling microdomains, where adenylyl cyclases, cAMP-degrading phosphodiesterases, cAMP effectors, and downstream substrates are brought together in one place to maintain specificity of cAMP signaling (Kamenetsky et al., 2006,

259 Lefkimmiatis \& Zaccolo, 2014). Our finding that SAC activity prevents rapid depletion of glycogen in 260 the absence of glucose suggests that cAMP from SAC exerts an effect that is opposite to that of the 261 well-established tmAC-cAMP-PKA axis. We confirmed these two opposing cAMP signaling pathways 262 in HepG2 cells by demonstrating that both stimulation of tmACs by forskolin and inhibition of sAC by LRE1 promoted glycogenolysis (Figure 5A and 5B). and from tmACs on glycogen. sAC has been shown to signal via both Epac (Flacke, Flacke et al., 2013, 
Onodera, Nam et al., 2014) and PKA (Acin-Perez et al., 2009, Valsecchi, Konrad et al., 2017). Since tmAC-derived cAMP regulates glycogenolysis via PKA, we hypothesized that sAC-derived cAMP uses a different cAMP effector, namely Epac1 or Epac2. In glucose-starved HepG2 cells, i.e., fueled with only octanoate (Figure 5C) or acutely starved (Figure 5D), inhibition of Epac1 by the specific inhibitor

271 (R)-CE3F4 (Courilleau, Bisserier et al., 2012) induced an acute, transient increase in ECAR with the 272 same temporal and dynamic characteristics as SAC inhibition (Figure $3 \mathrm{~F}$ and $3 \mathrm{H}$ ), suggesting that

273 Epac1 mediates SAC-dependent regulation of glycogenolysis. In contrast, the Epac2-specific inhibitor ESI-05 (Tsalkova, Mei et al., 2012) and the PKA inhibitor H89 did not induce significant ECAR changes.

To confirm the observed ECAR changes, we examined how Epac1, Epac2, and PKA regulate glycogenolysis by measuring the secretion of glycolytic end products and cellular glycogen levels. Indeed, in HepG2 cells acutely starved of glucose, only inhibition of Epac1 phenocopied the sACsuppressed metabolic phenotype, which included increased lactate secretion, decreased pyruvate secretion, and increased medium lactate-to-pyruvate ratio (Figure S5A-S5C). Consistently, the Epac1specific inhibitor (R)-CE3F4, but not the Epac2-specific inhibitor ESI-05 or the PKA inhibitor H89, induced significant glycogen breakdown in the absence of glucose in both HepG2 and H69 (Figure $5 E$ and $5 F$, respectively). In addition, the PKA-selective activator dibutyryl-cAMP also promoted glycogen breakdown in both cell lines, confirming that PKA and Epac1 mediate opposite metabolic effects of tmAC and SAC signaling, respectively. Moreover, we observed that in the presence of glucose, the Epac1-specific inhibitor (R)-CE3F4 induced significant glycogen breakdown in H69 but not in HepG2 cells (Figure $5 \mathrm{G}$ and $5 \mathrm{H}$ ), which mirrored the differential effects of sAC inhibition on glycogen in these cells (Figure $4 \mathrm{~A}$ and $4 \mathrm{~B}$ ). Similarly, inhibition of SAC or Epac1 also induced glycogenolysis in several other cell lines (Figure S5G-S5I).

Glycogen content in cells is under the control of glycogen synthase and glycogen phosphorylase. Since inhibiting SAC-CAMP-Epac1 signaling promotes glycogenolysis in the absence of glucose, where glycogen synthase lacks substrates, we tested whether inhibition of SAC-CAMP-Epac1 signaling would increase glycogen phosphorylase activity. The activity of glycogen phosphorylase is regulated allosterically by AMP and covalently by phosphorylation of Ser15 by phosphorylase kinase. Since SAC inhibition did not cause changes in the adenylate energy charge in cytosol, we investigated if SACCAMP-Epac1 regulates glycogen phosphorylase by affecting its phosphorylation status at Ser15. We investigated this possibility in $\mathrm{H69}$ cells because SAC-CAMP-Epac1 regulates the glycogen content of H69 cells also in the presence of a physiologic concentration of glucose. Indeed, both SAC-specific inhibitor LRE1 as well as tmAC-specific activator forskolin acutely increased Ser15 phosphorylation 
of glycogen phosphorylase (PYGL) (Figure 5I). Similarly, both the Epac1-selective inhibitor (R)-CE3F4 and the PKA-selective activator dibutyryl-cAMP induced acute phosphorylation of Ser15 in PYGL (Figure 5J). These data show that SAC-derived CAMP and tmAC-derived cAMP define independent signaling microdomains that have opposite effects on glycogenolysis: SAC-cAMP-Epac1 signaling suppresses glycogenolysis while tmAC-cAMP-PKA signaling promotes glycogenolysis.

\section{Discussion}

Our present study demonstrates that the evolutionarily conserved SAC, which generates CAMP proportionately to intracellular levels of ATP, bicarbonate and free $\mathrm{Ca}^{2+}$, regulates the acute switch between aerobic glycolysis and glycogenolysis on the one hand and oxidative phosphorylation on the other (Figure 6). SAC-derived cAMP regulates complex I flux and ATP generation by oxidative phosphorylation; at the same time, and independently, SAC-derived CAMP signals via Epac1 to maintain glycogen levels. Via this metabolic tuning, SAC regulates the relative bioenergetic contributions of glycolysis, glycogenolysis, and oxidative phosphorylation to maintain a constant overall ATP production rate under different substrate availability and thereby maintains the adenylate energy charge.

The primary metabolic signature of the SAC-suppressed status, as examined by pharmacological and genetic suppression of $S A C$, is a Warburg-like metabolic phenotype consisting of increased aerobic glycolysis, elevated cytosolic $\mathrm{NADH} / \mathrm{NAD}^{+}$ratio, and suppressed oxidative phosphorylation. In all cell lines tested and primary mouse hepatocytes, SAC inhibition acutely increased lactate secretion but decreased pyruvate secretion. The resulting increased lactate-to-pyruvate ratio in medium reflects an increase in the cytosolic $\mathrm{NADH} / \mathrm{NAD}{ }^{+}$ratio, which was confirmed by experiments with the NADH/NAD ${ }^{+}$biosensor Peredox. These data are consistent with the long-standing concept that the cytosolic $\mathrm{NADH} / \mathrm{NAD}^{+}$ratio can be inferred from the corresponding substrate-product pair of LDH (Veech, Raijman et al., 1970) and shows that SAC is a general regulator of the cytosolic $\mathrm{NADH} / \mathrm{NAD}^{+}$redox state. Most likely, sAC regulates the cytosolic NADH/NAD ${ }^{+}$redox state by affecting complex I of electron transport chain, as evidenced by the results of our experiments with digitoninpermeabilized cells. 
simultaneously and stably increased the ECAR, elevated the cytosolic NADH/NAD ${ }^{+}$ratio, and decreased the OCR. When glucose was removed from the medium, all the metabolic changes caused by SAC inhibition became transient, even if the cells were fueled with octanoate. Importantly, the transient changes in ECAR could be abolished by 2-DG and CP-91149, suggesting that the rise in $\mathrm{NADH} / \mathrm{NAD}^{+}$ratio by SAC inhibition is supported by glycogenolysis. Of note, the 2-DG and CP-91149 also greatly reduced the suppression of OCR by the SAC inhibitor, suggesting that the transient glycolytic flux from glycogenolysis also contributed to the suppression of oxidative phosphorylation. Importantly, these reciprocal changes in glycolysis and oxidative phosphorylation did not result in change of ATP production rate or the cytosolic adenylate energy charge, suggesting that SAC is a bioenergetic switch. In concordance with our data, Valsecchi et al reported that sAC knockout mouse embryonic fibroblasts also have increased ECAR and reduced oxidative phosphorylation (Valsecchi et al., 2017). They attributed this to reduced complex I activity in SAC knockout cells, while our data reveal that SAC regulates both complex I and glycogenolysis via independent mechanisms. In this way, SAC maintains bioenergetic homeostasis both in the presence of glucose and under acute glucose starvation.

The third and most important feature of the metabolic changes induced by SAC inhibition is a striking induction of glycogenolysis. Cellular glycogen levels are regulated by glycogen synthase and glycogen phosphorylase, both of which are regulated by phosphorylation as well as allosterically. Phosphorylation of glycogen synthase at serine residues 641,645 , and 649 by glycogen synthase kinase inactivates the enzyme (Roach, 1990). Glycogen phosphorylase exists in two states: the active

$354 \quad \mathrm{R}$ state and the much less active $\mathrm{T}$ state. Phosphorylation at serine 15 by phosphorylase kinase 355 converts phosphorylase $b$ to phosphorylase $a$, which has a favorable equilibrium for the active $\mathrm{R}$ state (Nolan, Novoa et al., 1964). These phosphorylation events are subject to simultaneous counterregulation by protein phosphatase 1 (Cohen, 1983). In addition, glycogen phosphorylase $a$ is allosterically inhibited by glucose while glycogen phosphorylase $b$ is allosterically stimulated by AMP and inhibited by glucose-6-phosphate and ATP (reviewed in (Johnson, 1992)). On the other hand, glycogen synthase is allosterically activated by glucose-6-phosphate (Bouskila, Hunter et al., 2010). In the presence of glucose, high intracellular glucose and glucose-6-phosphate levels suppress stimulates glycogen synthase activity. Therefore, depending on glycogen synthase activity, the net result of suppression of SAC-CAMP-Epac1 in the presence of glucose may have variable effects on glycogen level in different types of cells. In the absence of glucose, glycogen synthase activity is reduced and inhibition of SAC-cAMP-Epac1 always results in rapid breakdown of glycogen, indicating increased glycogen phosphorylase activity. Our result show that inhibition of SAC-cAMP-Epac1 
indeed promoted the conversion of phosphorylase $b$ to phosphorylase $a$, which can be the result of either increased phosphorylase kinase activity or reduced protein phosphatase activity. Because SAC inhibition does not affect the adenylate energy charge of the cells, SAC-cAMP-Epac1 signaling is unlikely to allosterically activate glycogen phosphorylase by increasing AMP or decreasing ATP.

Our data demonstrate in cells of different tissue origins that cellular glycogen store is regulated by a novel SAC-CAMP-Epac1 signaling pathway that suppresses glycogenolysis. In contrast, the mobilization of glycogen store by epinephrine in muscle and by glucagon in liver both engage the classic tmAC-cAMP-PKA signaling. While the hormone-stimulated tmAC-CAMP-PKA mediates glycogenolysis in specialized tissues, the SAC-CAMP-Epac1 signaling seems to be more widely present and suppresses glycogenolysis and aerobic glycolysis to preserving glycogen store. From the view point of autonomous cellular metabolism, the SAC-CAMP-Epac1 would facilitate complete utilization of glycogen for ATP generation by both glycolysis and oxidative phosphorylation during glucose starvation.

With the discovery of multiple hormones that utilize the GPCR-G ${ }_{\alpha s}$-tmAC-cAMP module for diverse cellular responses and the discovery of the intracellularly localized SAC, it is proposed that CAMP signaling is compartmentalized into microdomains to maintain specificity (Kamenetsky et al., 2006, Lefkimmiatis \& Zaccolo, 2014). Cyclic AMP generated by sAC and by tmACs, albeit free to diffuse, is buffered by regulatory units of PKA(Agarwal, Clancy et al., 2016) and constantly degraded by phosphodiesterases (PDEs)(Jurevicius \& Fischmeister, 1996, Zaccolo \& Pozzan, 2002). The spatial specificity is further enhanced by A-kinase anchoring proteins (AKAPs), a family of diverse scaffolding proteins that assemble adenylyl cyclases, PDEs, PKA, protein phosphatase, and protein substrates on defined locations (Beene \& Scott, 2007, Pidoux \& Tasken, 2010). In addition, membranes impermeable to cAMP, such as the mitochondrial inner membrane(Di Benedetto et al., 2013), and lipid rafts in plasma membrane (Agarwal, Yang et al., 2014) also contribute to the spatial specificity. These mechanisms probably all contribute to the observed opposite effects of SAC and tmACs. For example, cAMP signaling by SAC promotes apoptosis (Chang et al., 2016, Kumar, Kostin et al., 2009) and compromises barrier function (Obiako, Calchary et al., 2013, Sayner, Alexeyev et al., 2006) while cAMP by tmACs protects against apoptosis (Chang et al., 2016, Kumar et al., 2009) and strengthens barrier function (Sayner et al., 2006). The differential signaling can also take place at the level of cAMP effectors. For instance, Epac1 activates Akt in a phosphatidylinositol 3-kinase-dependent manner while PKA suppresses Akt activation (Mei, Qiao et al., 2002). Our finding that SAC-CAMP- 
401 yet another evidence for the cAMP microdomain signaling model.

Our findings also suggest that the metabolic regulation by SAC is potentially important in physiologic and pathologic processes associated with a switch of ATP production between glycolysis and oxidative phosphorylation, such as cancer metabolism (DeBerardinis \& Chandel, 2016, Pavlova

406 \& Thompson, 2016), proliferation and differentiation of stem cells (Zhang, Nuebel et al., 2012), and

407 activation of macrophages, dendritic cells and T-cells (Kelly \& O'Neill, 2015). In particular, the 408 metabolic reprogramming in the SAC-suppressed state, namely increased glycolytic flux and 409 cytosolic $\mathrm{NADH} / \mathrm{NAD}^{+}$ratio with reduction in oxidative phosphorylation, resembles the seminal 410 observation described by Otto Warburg that growing tumors utilized glucose by aerobic glycolysis 411 despite sufficient oxygen tension for oxidative phosphorylation. This contention is supported by the observation that a large panel tumor samples have lower SAC expression than the corresponding

413 normal tissues (Ramos-Espiritu et al., 2016a). Moreover, the recent development of NADH/NAD ${ }^{+}$ 414 biosensors confirms that transformed cells have in general an elevated cytosolic free NADH/NAD ${ }^{+}$ 415 ratio as compared to non-transformed cells (Schwartz, Passonneau et al., 1974, Zhao, Hu et al., 2015). 416 While the constitutive Warburg effect in tumor cells is primarily driven by sustained genetic and 417 epigenetic changes, we show here that SAC also acutely regulates energy metabolism and the

418 Warburg-like metabolic phenotype. This mechanism may serve as a new therapeutic target to 419 manipulate proliferation of cancer cells and inflammatory responses of macrophages, dendritic cells, 420 and T-cells. 423 master regulator of cellular energy metabolism, including the cytosolic $\mathrm{NADH} / \mathrm{NAD}^{+}$redox state, 424 glycolysis, oxidative phosphorylation and glycogenolysis. It acts as a metabolic switch that may be 425 explored for its therapeutic value in pathologic processes characterized by aberrant metabolic reprogramming. 


\section{Acknowledgements}

430 This study was supported by grant \#11652-2018-1 from the Dutch Cancer Foundation (KWF/Alpe

431 d'HuZes). The authors thank Dr. D. de Korte (Sanquin Blood Foundation, Amsterdam, NL) for

432 performing the analysis of adenylate nucleotides. Dr. Buck and Levin are supported by NIH grants

433 AG061290 and HD088571.

434

\section{Conflict of Interest statement}

436 Drs. Buck and Levin own equity interest in CEP Biotech which has licensed commercialization of a 437 panel of monoclonal antibodies directed against SAC.

438

\section{Author Contributions}

440 JCC, AJV and ROE developed the study concept. JCC, SG, EHG, HLL, HSH, and AJV designed, 441 performed, and analyzed the experiments. JCC drafted the manuscript. All co-authors commented 442 and revised the manuscript.

443 


\section{References}

Acin-Perez R, Salazar E, Kamenetsky M, Buck J, Levin LR, Manfredi G (2009) Cyclic AMP produced inside mitochondria regulates oxidative phosphorylation. Cell Metab 9: 265-276

Affourtit C, Brand MD (2009) Measuring mitochondrial bioenergetics in INS-1E insulinoma cells. Methods Enzymol 457: 405-24

Agarwal SR, Clancy CE, Harvey RD (2016) Mechanisms Restricting Diffusion of Intracellular cAMP. Sci Rep 6: 19577

Agarwal SR, Yang PC, Rice M, Singer CA, Nikolaev VO, Lohse MJ, Clancy CE, Harvey RD (2014) Role of membrane microdomains in compartmentation of CAMP signaling. PLoS One 9: e95835

Atkinson DE, Walton GM (1967) Adenosine triphosphate conservation in metabolic regulation. Rat liver citrate cleavage enzyme. J Biol Chem 242: 3239-41

Beene DL, Scott JD (2007) A-kinase anchoring proteins take shape. Curr Opin Cell Biol 19: 192-8

Bontekoe IJ, van der Meer PF, van den Hurk K, Verhoeven AJ, de Korte D (2017) Platelet storage performance is consistent by donor: a pilot study comparing "good" and "poor" storing platelets. Transfusion 57: 2373-2380

Bouskila M, Hunter RW, Ibrahim AF, Delattre L, Peggie M, van Diepen JA, Voshol PJ, Jensen J, Sakamoto $\mathrm{K}$ (2010) Allosteric regulation of glycogen synthase controls glycogen synthesis in muscle. Cell Metab 12: $456-66$

Chang JC, Go S, de Waart DR, Munoz-Garrido P, Beuers U, Paulusma CC, Oude Elferink R (2016) Soluble Adenylyl Cyclase Regulates Bile Salt-Induced Apoptosis in Human Cholangiocytes. Hepatology 64: 52234

Chen Y, Cann MJ, Litvin TN, lourgenko V, Sinclair ML, Levin LR, Buck J (2000) Soluble adenylyl cyclase as an evolutionarily conserved bicarbonate sensor. Science 289: 625-628

Cohen $\mathrm{P}$ (1983) Protein phosphorylation and the control of glycogen metabolism in skeletal muscle. Philos Trans R Soc Lond B Biol Sci 302: 13-25

Courilleau D, Bisserier M, Jullian JC, Lucas A, Bouyssou P, Fischmeister R, Blondeau JP, Lezoualc'h F (2012) Identification of a tetrahydroquinoline analog as a pharmacological inhibitor of the cAMPbinding protein Epac. J Biol Chem 287: 44192-202

De Rasmo D, Micelli L, Santeramo A, Signorile A, Lattanzio P, Papa S (2016) cAMP regulates the functional activity, coupling efficiency and structural organization of mammalian FOF1 ATP synthase. Biochim Biophys Acta 1857: 350-8

De Rasmo D, Signorile A, Santeramo A, Larizza M, Lattanzio P, Capitanio G, Papa S (2015) Intramitochondrial adenylyl cyclase controls the turnover of nuclear-encoded subunits and activity of mammalian complex I of the respiratory chain. Biochim Biophys Acta 1853: 183-91

DeBerardinis RJ, Chandel NS (2016) Fundamentals of cancer metabolism. Sci Adv 2: e1600200

Di Benedetto G, Scalzotto E, Mongillo M, Pozzan T (2013) Mitochondrial Ca(2)(+) uptake induces cyclic AMP generation in the matrix and modulates organelle ATP levels. Cell Metab 17: 965-75 
481 Flacke JP, Flacke H, Appukuttan A, Palisaar RJ, Noldus J, Robinson BD, Reusch HP, Zippin JH, Ladilov Y
482 (2013) Type 10 soluble adenylyl cyclase is overexpressed in prostate carcinoma and controls

482

483

484

485

486

487

488

489

490

491 proliferation of prostate cancer cells. J Biol Chem 288: 3126-35

Geng W, Wang Z, Zhang J, Reed BY, Pak CY, Moe OW (2005) Cloning and characterization of the human soluble adenylyl cyclase. Am J Physiol Cell Physiol 288: C1305-16

Gilglioni E.H. CJC, Duijst S., Go S., Adam A.A., Hoekstra R., Verhoeven A.J. Ishii-Iwamoto E.L., Oude ElferinkR.P. (2018) Improved oxygenation dramatically alters metabolism and gene expression in cultured primary mouse hepatocytes. Hepatology Communications 2: 299-312

Grubman SA, Perrone RD, Lee DW, Murray SL, Rogers LC, Wolkoff LI, Mulberg AE, Cherington V, Jefferson DM (1994) Regulation of intracellular $\mathrm{pH}$ by immortalized human intrahepatic biliary epithelial cell lines. Am J Physiol 266: G1060-70

Halestrap AP, Wilson MC (2012) The monocarboxylate transporter family--role and regulation. IUBMB Life 64: 109-19

Han H, Stessin A, Roberts J, Hess K, Gautam N, Kamenetsky M, Lou O, Hyde E, Nathan N, Muller WA, Buck J, Levin LR, Nathan C (2005) Calcium-sensing soluble adenylyl cyclase mediates TNF signal transduction in human neutrophils. J Exp Med 202: 353-61

Hung YP, Albeck JG, Tantama M, Yellen G (2011) Imaging cytosolic NADH-NAD(+) redox state with a genetically encoded fluorescent biosensor. Cell Metab 14: 545-54

Jaiswal BS, Conti M (2003) Calcium regulation of the soluble adenylyl cyclase expressed in mammalian spermatozoa. Proc Natl Acad Sci U S A 100: 10676-81

Johnson LN (1992) Glycogen phosphorylase: control by phosphorylation and allosteric effectors. FASEB J 6: $2274-82$

Jurevicius J, Fischmeister R (1996) cAMP compartmentation is responsible for a local activation of cardiac Ca2+ channels by beta-adrenergic agonists. Proc Natl Acad Sci U S A 93: 295-9

Kamenetsky M, Middelhaufe S, Bank EM, Levin LR, Buck J, Steegborn C (2006) Molecular details of cAMP generation in mammalian cells: a tale of two systems. J Mol Biol 362: 623-39

Katona D, Rajki A, Di Benedetto G, Pozzan T, Spat A (2015) Calcium-dependent mitochondrial cAMP production enhances aldosterone secretion. Mol Cell Endocrinol 412: 196-204

Kelly B, O'Neill LA (2015) Metabolic reprogramming in macrophages and dendritic cells in innate immunity. Cell Res 25: 771-84

Kleinboelting S, Diaz A, Moniot S, van den Heuvel J, Weyand M, Levin LR, Buck J, Steegborn C (2014) Crystal structures of human soluble adenylyl cyclase reveal mechanisms of catalysis and of its activation through bicarbonate. Proc Natl Acad Sci U S A 111: 3727-32

Kobayashi M, Buck J, Levin LR (2004) Conservation of functional domain structure in bicarbonateregulated "soluble" adenylyl cyclases in bacteria and eukaryotes. Dev Genes Evol 214: 503-9

Kumar S, Kostin S, Flacke JP, Reusch HP, Ladilov Y (2009) Soluble adenylyl cyclase controls mitochondria-dependent apoptosis in coronary endothelial cells. J Biol Chem 284: 14760-14768

Lefkimmiatis K, Zaccolo M (2014) cAMP signaling in subcellular compartments. Pharmacol Ther 143: 295-304

Levin LR, Buck J (2015) Physiological roles of acid-base sensors. Annu Rev Physiol 77: 347-62 
521 Litvin TN, Kamenetsky M, Zarifyan A, Buck J, Levin LR (2003) Kinetic properties of "soluble" adenylyl

522 cyclase. Synergism between calcium and bicarbonate. J Biol Chem 278: 15922-6

523

524

525

526

527

528

529

530

531

532

533

534

535

536

537

538

539

540

541

542

543

544

545

546

547

548

549

550

551

552

553

554

555

556

557

558

559

560

Mei FC, Qiao J, Tsygankova OM, Meinkoth JL, Quilliam LA, Cheng X (2002) Differential signaling of cyclic AMP: opposing effects of exchange protein directly activated by cyclic AMP and cAMPdependent protein kinase on protein kinase B activation. J Biol Chem 277: 11497-504

Mookerjee SA, Gerencser AA, Nicholls DG, Brand MD (2017) Quantifying intracellular rates of glycolytic and oxidative ATP production and consumption using extracellular flux measurements. J Biol Chem 292: 7189-7207

Mookerjee SA, Goncalves RLS, Gerencser AA, Nicholls DG, Brand MD (2015) The contributions of respiration and glycolysis to extracellular acid production. Biochim Biophys Acta 1847: 171-181

Nolan C, Novoa WB, Krebs EG, Fischer EH (1964) Further Studies on the Site Phosphorylated in the Phosphorylase B to a Reaction. Biochemistry 3: 542-51

Obiako B, Calchary W, Xu N, Kunstadt R, Richardson B, Nix J, Sayner SL (2013) Bicarbonate disruption of the pulmonary endothelial barrier via activation of endogenous soluble adenylyl cyclase, isoform 10. Am J Physiol Lung Cell Mol Physiol 305: L185-L192

Onodera Y, Nam JM, Bissell MJ (2014) Increased sugar uptake promotes oncogenesis via EPAC/RAP1 and O-GlcNAc pathways. J Clin Invest 124: 367-84

Pavlova NN, Thompson CB (2016) The Emerging Hallmarks of Cancer Metabolism. Cell Metab 23: 2747

Pidoux G, Tasken K (2010) Specificity and spatial dynamics of protein kinase A signaling organized by A-kinase-anchoring proteins. J Mol Endocrinol 44: 271-84

Ramos-Espiritu L, Diaz A, Nardin C, Saviola AJ, Shaw F, Plitt T, Yang X, Wolchok J, Pirog EC, Desman G, Sboner A, Zhang T, Xiang J, Merghoub T, Levin LR, Buck J, Zippin JH (2016a) The metabolic/pH sensor soluble adenylyl cyclase is a tumor suppressor protein. Oncotarget 7: 45597-45607

Ramos-Espiritu L, Kleinboelting S, Navarrete FA, Alvau A, Visconti PE, Valsecchi F, Starkov A, Manfredi G, Buck H, Adura C, Zippin JH, van den Heuvel J, Glickman JF, Steegborn C, Levin LR, Buck J (2016b) Discovery of LRE1 as a specific and allosteric inhibitor of soluble adenylyl cyclase. Nat Chem Biol 12: 838-44

Roach PJ (1990) Control of glycogen synthase by hierarchal protein phosphorylation. FASEB J 4: 2961-8

Rousset M, Zweibaum A, Fogh J (1981) Presence of glycogen and growth-related variations in 58 cultured human tumor cell lines of various tissue origins. Cancer Res 41: 1165-70

Salabei JK, Gibb AA, Hill BG (2014) Comprehensive measurement of respiratory activity in permeabilized cells using extracellular flux analysis. Nat Protoc 9: 421-38

Sayner SL, Alexeyev M, Dessauer CW, Stevens T (2006) Soluble adenylyl cyclase reveals the significance of cAMP compartmentation on pulmonary microvascular endothelial cell barrier. Circ Res 98: 675-681

Schwartz JP, Passonneau JV, Johnson GS, Pastan I (1974) The effect of growth conditions on NAD+ and NADH concentrations and the NAD+:NADH ratio in normal and transformed fibroblasts. J Biol Chem 249: $4138-43$

Sutherland EW, Rall TW (1958) Fractionation and characterization of a cyclic adenine ribonucleotide formed by tissue particles. J Biol Chem 232: 1077-91 
561 Tsalkova T, Mei FC, Li S, Chepurny OG, Leech CA, Liu T, Holz GG, Woods VL, Jr., Cheng X (2012) Isoform562 specific antagonists of exchange proteins directly activated by cAMP. Proc Natl Acad Sci U S A 109: $563 \quad 18613-8$

564 Urbansky ET, Schock MR (2000) Understanding, deriving, and computing buffer capacity. J Chem Educ 565 77: 1640-1644

566 Valsecchi F, Konrad C, D'Aurelio M, Ramos-Espiritu LS, Stepanova A, Burstein SR, Galkin A, Magrane J, 567 Starkov A, Buck J, Levin LR, Manfredi G (2017) Distinct intracellular sAC-cAMP domains regulate ER 568 Ca2+ signaling and OXPHOS function. J Cell Sci 130: 3713-3727

569 Veech RL, Raijman L, Krebs HA (1970) Equilibrium relations between the cytoplasmic adenine 570 nucleotide system and nicotinamide-adenine nucleotide system in rat liver. Biochem J 117: 499-503

571 Zaccolo M, Pozzan T (2002) Discrete microdomains with high concentration of cAMP in stimulated rat 572 neonatal cardiac myocytes. Science 295: 1711-5

573 Zhang J, Nuebel E, Daley GQ, Koehler CM, Teitell MA (2012) Metabolic regulation in pluripotent stem 574 cells during reprogramming and self-renewal. Cell Stem Cell 11: 589-95

575 Zhao Y, Hu Q, Cheng F, Su N, Wang A, Zou Y, Hu H, Chen X, Zhou HM, Huang X, Yang K, Zhu Q, Wang X, 576 Yi J, Zhu L, Qian X, Chen L, Tang Y, Loscalzo J, Yang Y (2015) SoNar, a Highly Responsive NAD+/NADH 577 Sensor, Allows High-Throughput Metabolic Screening of Anti-tumor Agents. Cell Metab 21: 777-89

578 Zippin JH, Chen Y, Straub SG, Hess KC, Diaz A, Lee D, Tso P, Holz GG, Sharp GW, Levin LR, Buck J (2013) $579 \mathrm{CO} / \mathrm{HCO}(-)$ - and calcium-regulated soluble adenylyl cyclase as a physiological ATP sensor. J Biol 580 Chem 288: 33283-91 


\section{Materials and Methods}

584

585

586

587

588

589

590

591

592

593

594

595

596

597

598

599

600

601

602

603

604

605

606

607

608

609

610

611

\section{Materials}

Unless otherwise indicated, materials were purchased from Sigma-Aldrich. Tet-pLKO-puro was a gift from Dmitri Wiederschain (Addgene plasmid \#21915). pLKO.1-puro was a gift from Bob Weinberg (Addgene plasmid \#8453). pCW-Cas9 was a gift from Eric Lander \& David Sabatini (Addgene plasmid \#50661). pcDNA3.1-Peredox-mCherry was a gift from Gary Yellen (Addgene plasmid \#32383).

\section{Methods}

\section{Cell culture and lentiviral transduction}

The immortalized human cholangiocytes H69 is kind gift from Douglas Jefferson (Grubman et al., 1994) and was cultured in DMEM/F-12 (3:1) with hormonal supplements as described (Chang et al., 2016). HepG2, Caco-2, B16F10, and Raw264.7 were cultured in DMEM (Invitrogen) with $2 \mathrm{~g} / \mathrm{L}$ glucose, $1.8 \mathrm{~g} / \mathrm{L} \mathrm{NaHCO}_{3}, 20 \mathrm{mM}$ HEPES-NaOH pH 7.4 and 10\% fetal bovine serum. For stable genetically modified cell lines, cells were transduced with lentivirus and selected with $2.5 \mu \mathrm{g} / \mathrm{mL}$ puromycin or $5 \mu \mathrm{g} / \mathrm{mL}$ basticidin. In acute transduction experiments, the transduced cells were used without puromycin selection. For all experiments, cells were refreshed the day before experiments.

\section{Isolation and culture of primary mouse hepatocytes}

Animal experiments were approved by the institutional animal experiment committee. Primary mouse hepatocytes were isolated from wild-type male C57BL/6 mice after overnight ad libitum feeding by a two-step collagenase perfusion method through the portal vein. Cells were cultured in collagen sandwich configuration overnight on a 60-rpm shaking platform in a $5 \% \mathrm{CO}_{2}, 37^{\circ} \mathrm{C}$ incubator. The isolation procedure and culture of primary mouse hepatocytes were performed as described (Gilglioni E.H., 2018).

\section{Construction of lentiviral vectors and lentiviral production}

The cloning procedure and short hairpin oligo sequences used to construct tetracycline- 
612 inducible lentiviral vector for SAC knockdown and scramble control has been described

613 previously (Chang et al., 2016). Using pLKO.1-puro, the same procedure was followed to

614 construct constitutively active lentiviral vectors for SAC knockdown and scramble control. To

615 avoid formation of intracellular aggregation, we subcloned Peredox-mCherry NADH/NAD ${ }^{+}$

616 redox sensor for inducible expression. Briefly, puromycin resistance gene of pCW-Cas9 was

617 removed by Hincll and Xbal. Blasticidin resistance gene (Bsd) was amplified by forward primer

618 5'-CAGTCGGCTCCCTCGTTGACCGAATCACCGA

619 CCTCTCTCCCCAGACGCGTATGGCCAAGCCTTTGTCTCA-3' and reverse primer 5'-

620 TTGTCCAGTCTAGACAT

621 TGGACCAGGGTTTTCTTCAACATCACCACAAGTGAGGAGAGAACCTCTACCTTCATGCATGCCCTCCCA

622 CACATAAC-3'. The purified PCR fragment was digested with Hincll and Xbal and ligated with

623 the open pCW-Cas9. The Cas9 insert was then removed by restriction enzymes Nhel and

624 BamHI. To create a multiple cloning site, sense oligo (5'-

625 CTAGCGTCGACACCGGTGAATTCGTTTAAACCCTGCAGGG-3') and antisense oligo (5'-

626 GATCCCCTGCAGGGTTTAAACGAATTCACCGGTGTCGACG-3') were annealed and ligated to the

627 pCW backbone for pCW-MCS-BSD. The Peredox-mCherry insert was prepared by digesting

628 pcDNA3.1-Peredox-mCherry with Xbal and Pmel. pCW-MCS-BSD was digested with Nhel and

629 Pmel and was ligated to the Peredox-mCherry insert for pCW-Peredox-mCherry-BSD.

630 Lentivirus was produced in HEK293T using the third-generation packaging system.

631

632 Sample preparation and enzymatic assays for medium metabolites

633 Cells were refreshed with full culture medium one day prior to the experiment. On the day

634 of the experiment, medium was changed to the experimental medium and cells were allowed

635 to equilibrate in the experimental medium for one hour. The experimental medium was DMEM

636 without glutamine, with $5.5 \mathrm{mM}$ glucose and 10\% (Figure 1) or 1\% (Figure S1, Figure 5B) fetal

637 bovine serum. In all other experiments, the experimental medium was based on modified

638 Hank's balanced salt solution (HBSS) for ambient air supplemented with $5.5 \mathrm{mM}$ glucose and

$6390.1 \%$ fatty acid-free bovine serum albumin (BSA) (please refer to Suppl. Table 1 for

640 formulation). We did not observe an effect of amino acids on the acute regulation of the

641 Warburg-like phenotype and glycogen metabolism by sAC. At the start of the experiment, cells

642 were exposed to media containing the indicated inhibitors and vehicle controls. At indicated

643 time points, $50 \mu \mathrm{L}$ spent medium was harvested and mixed with $75 \mu \mathrm{L}$ ice-cold $5 \%(\mathrm{w} / \mathrm{v})$

644 metaphosphoric acid (MPA) for deproteinization. After incubating for at least one hour at $4^{\circ} \mathrm{C}$, 
the MPA-acidified samples were centrifuged at $20,000 \times \mathrm{g}$ for 10 minutes. The supernatants

646

647

648

649

650

651

652

653

654

655

656

657

658

659

660

661

662

663

664

665

666

667

668

669

670

671

672

673

674

675

676

were harvested for enzymatic determination of L-lactate, pyruvate, and glucose. For the determination of intracellular lactate, pyruvate, and glucose-6-phosphate, medium was aspirated. Cells were washed once with ice-cold PBS and lysed in 3\% ice-cold MPA. The acid homogenates were incubated at least one hour at $4^{\circ} \mathrm{C}$ and centrifuged at $20,000 \times \mathrm{g}$ for 10 minutes at $4^{\circ} \mathrm{C}$. Lactate, pyruvate, and glucose are stable in $3 \%$ MPA at $4^{\circ} \mathrm{C}$ and were assayed directly from the MPA extracts.

\section{Enzymatic assay for L-lactate, pyruvate, glucose}

The enzymatic determinations of L-lactate, pyruvate and glucose were performed as previously described (Gilglioni E.H., 2018).

\section{Sample preparation and enzymatic determination of glycogen}

At the end of the incubation, cells were washed twice with ice-cold PBS and lysed in TTE buffer (1\% Triton X-100, 10 mM Tris-HCl pH 8.0 and 1 mM EDTA-NaOH, pH 8.0) or RIPA buffer (150 mM NaCl, 20 mM Tris-HCl pH 8.0, 1\% Triton X-100, 0.1\% SDS, 0.5\% Na-deoxycholate). Lysates were centrifuged at $20,000 \times \mathrm{g}$ at $4^{\circ} \mathrm{C}$ for 10 minutes. Supernatants were harvested for determination of glycogen and protein concentration. For the determination of glycogen, 50 $\mu \mathrm{L}$ of supernatant was mixed with $20 \mu \mathrm{L} 0.35 \mathrm{M} \mathrm{NaOH}$ and heated at $80^{\circ} \mathrm{C}$ for 30 minutes to degrade monosaccharides. The hot alkali-treated lysates were then deproteinized by adding $30 \mu \mathrm{L} 10 \%(\mathrm{w} / \mathrm{v}) \mathrm{MPA}$ and incubated on ice for at least 1 hour or overnight at $4^{\circ} \mathrm{C}$. Samples were centrifuged at $20,000 \times \mathrm{g}$ for 10 minutes. Twenty microliters of deproteinized samples or glucose standards (prepared in 3\% MPA) were mixed with $100 \mu \mathrm{L}$ solution A $(0.75 \mathrm{mM}$ homovanillic acid, $2.5 \mathrm{U} / \mathrm{mL}$ amyloglucosidase from Aspergillus niger, $50 \mathrm{mM} \mathrm{K}{ }_{2} \mathrm{HPO}_{4}-\mathrm{KH}_{2} \mathrm{PO}_{4}$, $\mathrm{pH}$ 8.0). The resulting mixture has a pH of about 4.7 , which is optimal for amyloglucosidase to hydrolyze glycogen. After 1-hour incubation at $45^{\circ} \mathrm{C}, 50 \mu \mathrm{L}$ solution B was added $(2 \mathrm{U} / \mathrm{L}$ horseradish peroxidase, $500 \mathrm{mM} \mathrm{K}_{2} \mathrm{HPO}_{4}-\mathrm{KH}_{2} \mathrm{PO}_{4}, \mathrm{pH} 8.0$ ) to correct $\mathrm{pH}$ to 6.8 (assay condition for glucose). After determining the background fluorescence at $\lambda_{\mathrm{ex}} / \lambda_{\mathrm{em}}=320 / 450 \mathrm{~nm}$ in the CLARIOstar microplate reader (BMG LABTECH, Ortenberg, Germany), $50 \mu \mathrm{L}$ start solution (8 $\mathrm{U} / \mathrm{mL}$ glucose oxidase) was added and fluorescence was followed every 2 minutes until the reaction was completed (within 1 hour). 
Determination of ATP, ADP, and AMP by high performance liquid chromatography (HPLC) for adenylate energy charges in cytosol

679 HepG2 cells were treated with $0.1 \%$ DMSO or $50 \mu \mathrm{M}$ sAC-specific inhibitor LRE1 in HBSS 680 containing indicated substrates. After indicated period of incubation, cytosolic metabolites 681 were extracted with permeabilization buffer $(120 \mathrm{mM} \mathrm{KCl}, 10 \mathrm{mM} \mathrm{NaCl}, 5 \mathrm{mM}$ EDTA, $20 \mathrm{mM}$ 682 HEPES, pH 7.1) with $100 \mu \mathrm{g} / \mathrm{mL}$ digitonin. Two hundred microliter of digitonin extracts were 683 mixed with $16 \mu \mathrm{L} \mathrm{70 \%} \mathrm{perchloric} \mathrm{acid} \mathrm{for} \mathrm{deproteinization} \mathrm{and} \mathrm{subsequently} \mathrm{neutralized} \mathrm{with}$ $6842.5 \mathrm{M} \mathrm{K}_{2} \mathrm{CO}_{3}$. The neutralized perchloric acid extracts of HepG2 cells were then analyzed for 685 AMP, ADP and ATP by high-performance liquid chromatography using a Partisphere SAX 686 column (Whatman International Ltd.) exactly as described (Bontekoe, van der Meer et al., 687 2017). Adenylate energy charge was defined as ([ATP] + $0.5 \times[$ ADP $]) /([A T P]+[A D P]+[$ AMP $])$ 688 according to Atkinson and Walton (Atkinson \& Walton, 1967).

\section{Cyclic AMP accumulation and ELISA}

691 H69 cholangiocytes were cultured in 24-well plates. Cells were allowed to equilibrate with 692 the indicated experimental medium for 1 hour and refreshed again with $200 \mu \mathrm{L}$ experimental 693 medium. Cells were pretreated with sAC-specific inhibitor KH7 or LRE1 ( $25 \mu \mathrm{L}$ 10X solution) for 69410 minutes and $500 \mu \mathrm{M}$ 3-isobutyl-1-methylxanthine ( $25 \mu \mathrm{L} 10 \mathrm{X}$ solution) was added to induce 695 accumulation of cAMP. Accumulation was terminated by adding $100 \mu \mathrm{L} 0.35 \mathrm{M} \mathrm{HCl} / 3.5 \%$ 696 Triton $\mathrm{X}-100$. The acidic homogenate was heated at $95^{\circ} \mathrm{C}$ for 10 minutes and centrifuged at $69720,000 \times \mathrm{g}$ for 10 minutes at $4^{\circ} \mathrm{C}$. The cAMP content in the supernatant was measured by the Direct CAMP ELISA kit (Enzo Life Sciences).

\section{SDS-PAGE and Western blotting}

701 Protein concentrations of whole cell lysates in RIPA buffer were quantified by BCA assay. Equal amount of protein $(40-50 \mu \mathrm{g})$ was subjected to SDS-PAGE, transferred to PVDF membranes by semi-dry blotting and blocked overnight in 5\% non-fat milk / PBST (phosphatebuffered saline with $0.05 \%(\mathrm{w} / \mathrm{v})$ Tween 20$)$ at $4^{\circ} \mathrm{C}$. For immunodetection, the PVDF membranes were incubated with primary antibody for 1 hour, washed 3 times with TBST (Trisbuffered saline with $0.05 \%(\mathrm{w} / \mathrm{v})$ Tween 20$)$, incubated with horseradish peroxidaseconjugated secondary antibody for 1 hour, and washed again 4 times with TBST. All antibodies 
The PVDF membrane was developed with homemade enhanced chemiluminescence reagents (100 mM Tris- $\mathrm{HCl} \mathrm{pH} \mathrm{8.5,} 1.25 \mathrm{mM}$ luminol, $0.2 \mathrm{mM}$ p-coumarin and freshly added $3 \mathrm{mM} \mathrm{H}_{2} \mathrm{O}_{2}$ ) and detected using the ImageQuant LAS 4000 (GE Healthcare Life Sciences). Please refer to Table S2 for the list of primary and secondary antibodies and dilution.

\section{Ratiometric fluorescence measurement of cytosolic NADH/NAD with Peredox-mCherry} biosensor

Stable HepG2 cell lines harboring an inducible Peredox-mCherry construct or an empty vector (pCW-MCS-BSD) were cultured in clear-bottomed 96-well black plate (Costar 3603). Cells were induced with $0.8 \mu \mathrm{g} / \mathrm{mL}$ doxycycline for 48 hours before the experiment. Experiments were performed in HBSS modified for ambient air, supplemented with $0.1 \%$ BSA and indicated substrates at $37^{\circ} \mathrm{C}$. For glycogen depletion, cells were starved in modified HBSS for $5 \% \mathrm{CO}_{2}$ without glucose for 4 hours. Fluorescence of Peredox $\left(\mathrm{F}_{1}\right)$ and mCherry $\left(\mathrm{F}_{2}\right)$ was monitored at $\lambda_{\mathrm{Ex} 1} / \lambda_{\mathrm{Em} 1}=400-20 / 535-70(\mathrm{~nm})$ and $\lambda_{\mathrm{Ex} 2} / \lambda_{\mathrm{Em} 2}=570-20 / 645-80(\mathrm{~nm})$, respectively, in the CLARIOstar microplate reader. Responsiveness of the Peredox-mCherry sensor was confirmed in each experiment with a series of redox clamping solutions: $5 \mathrm{mM}$ L-lactate, $5 \mathrm{mM}$ pyruvate, and $5 \mathrm{mM} \mathrm{L-lactate/pyruvate} \mathrm{mixture} \mathrm{at} \mathrm{a} \mathrm{ratio} \mathrm{of} \mathrm{1,} \mathrm{5,} \mathrm{10,} \mathrm{20,} \mathrm{50,} \mathrm{and} 100$. Signals from cells transduced with the empty vector $\left(\mathrm{F}_{1}{ }^{\prime}\right.$ and $\mathrm{F}_{2}{ }^{\prime}$ for Peredox channel and mCherry channel, respectively) served to correct background. Background-corrected fluorescence ratio $R$ was defined as $\left(F_{1}-F_{1}{ }^{\prime}\right) /\left(F_{2}-F_{2}{ }^{\prime}\right)$. $R$ was linearly transformed by setting the minimal fluorescence ratio $R_{\min }$ ( $5 \mathrm{mM}$ pyruvate) and maximal fluorescence ratio $R_{\max }$ ( $5 \mathrm{mM}$ L-lactate) to 1 and 2 , respectively.

\section{Extracellular flux analysis}

Extracellular acidification rate (ECAR) and oxygen consumption rate (OCR) were measured with a Seahorse XF96 Analyzer (Agilent, United States). Cells were cultured in a 96-well Seahorse culture plate until confluence. Media were refreshed the day before the experiment. Cells were incubated 1 hour in experimental medium (modified HBSS for ambient air with $0.1 \%$ fatty acid-free BSA (see suppl. Table 1), with indicated substrates or inhibitors (2-deoxyglucose and glycogen phosphorylase $a$ inhibitor (P-91149) in ambient air at $37^{\circ} \mathrm{C}$ and then refreshed with the same medium immediately before determination of ECAR and OCR. Twenty-five microliters of concentrated compound solutions prepared in experimental medium were 
741 injected as indicated. The final concentrations of compounds were as follows: LRE1, $50 \mu \mathrm{M}$; oligomycin A, $1.6 \mu \mathrm{M}$; FCCP, $1.1 \mu \mathrm{M}$; antimycin A, $4.62 \mu \mathrm{M}$; rotenone, $2.31 \mu \mathrm{M}$. The coupled respiration rate was defined as the difference between the average of the first three OCR measurements after the addition of inhibitors or vehicle control and the last OCR measurement after the addition of oligomycin A. The FCCP-driven respiration rate was defined as the difference between the first OCR measurement after FCCP addition and the last OCR measurement after the addition of oligomycin A.

Estimation of ATP production by glycolysis and by mitochondria using extracellular flux measurements

751 The time-lapsed ATP production rates were calculated from extracellular fluxes (ECARs and OCRs) as elegantly described by Mookerjee et al. (Mookerjee et al., 2017, Mookerjee et al., 753 2015). Since cells were pre-incubated in HBSS with glucose or octanoate alone until reaching 754 steady state before imposing the acute perturbation of SAC activity, it was assumed that the oxidation of the provided substrates in the mitochondria was complete and that the contribution from other endogenous substrates (except for glycogen) was negligible. In cells fueled solely with octanoate, the glycolytic flux was assumed to come from glycogenolysis. The total ATP production rate $\left(J_{\text {ATP_total }}\right)$ is defined as the sum of the ATP production rate of glycolysis (JaTp_glycolysis, substrate level phosphorylation by phosphoglycerate kinase and 760 pyruvate kinase) and the ATP production rate of mitochondria ( $J_{\text {ATP_mitochondria, }}$ substrate level 761 phosphorylation by succinyl-CoA synthetase in the TCA cycle and oxidative phosphorylation 762 by the electron transport chain and ATP synthase). To calculate $J_{\text {ATP_glycolysis, }}$ it is necessary to distinguish the proton production by glycolysis from that of mitochondrial respiration. For this purpose, the total proton production rate at measurement time $t, \mathrm{PPR}_{\text {total }}(t)$, is derived from ECAR by the following equation:

$\operatorname{PPR}_{\text {total }}(t)=\operatorname{ECAR}(t) \times V \times \mathscr{B}$

767 where $\operatorname{ECAR}(t)$ is the ECAR at measurement time $t, V$ is the volume of the measurement micro768 chamber ( $2 \mu \mathrm{L}$ for Seahorse XF96 analyzer), and $\mathscr{B}$ is the buffer capacity of the experimental 769 medium. Since the experimental medium was buffered with 20mM HEPES-NaOH (pH 7.4), the 770 contribution of other salt components and $0.1 \%$ BSA to buffer capacity were negligible. Taken 771 the value of 7.31 as the $\mathrm{p} K_{a}$ of HEPES at $37^{\circ} \mathrm{C}$ and an average assay $\mathrm{pH}$ of 7.35 , the buffer 772 capacity $\mathscr{B}(\mathrm{M} / \mathrm{pH})$ of the experimental medium was calculated to be $1.31 \times 10^{-2} \mathrm{M} / \mathrm{pH}$ 
773 according to the definition of the buffer capacity (Urbansky \& Schock, 2000):

775

$$
\mathscr{B}=\frac{d C_{b}}{d(\mathrm{pH})}=\ln 10\left(\frac{K_{\mathrm{w}}}{\left[\mathrm{H}^{+}\right]}+\left[\mathrm{H}^{+}\right]+[\mathrm{X}]_{\mathrm{T}} \times \frac{K_{\mathrm{a}}\left[\mathrm{H}^{+}\right]}{\left(K_{\mathrm{a}}+\left[\mathrm{H}^{+}\right]\right)^{2}}\right)
$$

774

776

where $\max \mathrm{H}^{+} / \mathrm{O}_{2}$ is the maximal proton production per $\mathrm{O}_{2}$ molecule consumed, and $\mathrm{p} K_{\mathrm{a} 1}$ is

$$
\operatorname{OCR}_{\text {coupled }}(t)=\left(\mathrm{OCR}(t)-\mathrm{OCR}_{\mathrm{OA}}\right) \times \frac{1}{1-H}
$$

where $C_{\mathrm{b}}$ represents the normality of the added base, $[\mathrm{X}]_{T}$ represents the concentration of buffer, $K_{w}$ represents ionization constant of $\mathrm{H}_{2} \mathrm{O}$, and $K_{\mathrm{a}}$ represents the acid dissociation constant of the buffer.

The proton production rate of mitochondrial respiration $\left(\mathrm{PPR}_{\text {mito }}\right)$ and glycolysis $\left(\mathrm{PPR} \mathrm{g}_{\mathrm{gly}}\right)$ at measurement time $t$ is calculated as follows:

$$
\operatorname{PPR}_{\text {total }}(t)=\operatorname{PPR}_{\text {gly }}(t)+\operatorname{PPR}_{\text {mito }}(t)
$$

$\operatorname{PPR}_{\text {mito }}(t)=\operatorname{OCR}_{\text {mito }}(t) \times\left(\max \mathrm{H}^{+} / \mathrm{O}_{2}\right) \times \frac{\left[\mathrm{HCO}_{3}^{-}\right]}{\left[\mathrm{CO}_{2}\right]+\left[\mathrm{HCO}_{3}^{-}\right]}$

$$
=\mathrm{OCR}_{\text {mito }}(t) \times\left(\max \mathrm{H}^{+} / \mathrm{O}_{2}\right) \times \frac{10^{\mathrm{pH}-\mathrm{p} K_{\mathrm{a} 1}}}{1+10^{\mathrm{pH}-\mathrm{p} K_{\mathrm{a} 1}}}
$$

$\operatorname{PPR}_{\text {gly }}(t)=\operatorname{PPR}_{\text {total }}(t)-\operatorname{PPR}_{\text {mito }}(t)$ dissociation of $\mathrm{H}_{2} \mathrm{CO}_{3}$. The $\mathrm{OCR}_{\text {mito }}$ (needed for the calculation of $\mathrm{PPR}_{\text {mito }}$ ) and $\mathrm{OCR}$ coupled (needed for the calculation of $J_{\text {ATP_mitochondria) }}$ at measurement time $t$ are derived from the measured $\mathrm{OCR}, \mathrm{OCR}(t)$, as follows:

where $O C R_{O A}$ and $O C R_{A+R}$ are the last of the three $O C R$ measurements after addition of oligomycin $A$ and the combination of antimycin $A$ and rotenone, respectively. Because

798 oligomycin A increases the mitochondria membrane potential, proton leak and the associated oxygen consumption will increase. This will lead to an underestimation of the coupled OCR. 
800

801

802

803

804

805

806

807

808

809

810

811

812

813

814

815

816

817

818

819

820

822

823

824

825

826

827

828

829

830

Therefore, a correction for hyperpolarisation is included, where $H$ represents the percentage of underestimation and is taken to be $9.2 \%$ according to Affourtit and Brand (Affourtit \& Brand,

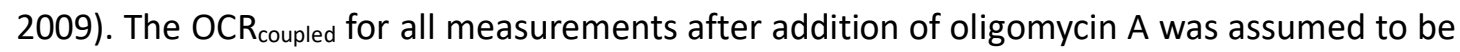
zero. Finally, the ATP production rates $J_{\text {ATP_total, }} J_{\text {ATP_glycolysis, }} J_{\text {ATP_oxphos, }} J_{\text {ATP_TCA, }}$ and $J_{\text {ATP_mitochondria }}$ at measurement time $t$ were calculated as follows:

$J_{\text {ATP_total }}(t)=J_{\text {ATP_glycolysis }}(t)+J_{\text {ATP_mitochondria }}(t)$

$J_{\text {ATP_glycolysis }}(t)=\mathrm{PPR}_{\text {gly }}(t) \times$ ATP/lactate $+2 \times \mathrm{OCR}_{\text {mito }}(t) \times \mathrm{P} / \mathrm{O}_{\text {gly }}$

$J_{\text {ATP_mitochondria }}(t)=J_{\text {ATP }_{\text {oxphos }}}(t)+J_{\text {ATP_TCA }_{\text {T }}}(t)$

$J_{\text {ATP_oxphos }}(t)=\mathrm{OCR}_{\text {coupled }}(t) \times \mathrm{P} / \mathrm{O}_{\text {oxphos }}$

$J_{\mathrm{ATP}_{-} \mathrm{TCA}}(t)=\mathrm{OCR}_{\text {mito }}(t) \times \mathrm{P} / \mathrm{O}_{\mathrm{TCA}}$

where ATP/lactate represents the ATP production per lactate produced. With glucose as the substrate, ATP/lactate is 1 . With glycogen as the substrate, assuming a composition of $90 \% \alpha$ 1,4-glycosidic bonds and $10 \% \alpha-1,6$-glycosidic bonds, the ATP/lactate is 1.45 . The lactate production rate is assumed to be equal to $\mathrm{PPR}$ gly. The values of $\max \mathrm{H}^{+} / \mathrm{O}_{2}$, ATP/lactate, $\mathrm{P} / \mathrm{O}_{\mathrm{gly}}$, $\mathrm{P} / \mathrm{O}_{\text {oxphos, }}$ and $\mathrm{P} / \mathrm{O}_{\text {TCA }}$ for no substrate (i.e. glycogen), glucose and octanoate were taken from or calculated as in the works of Mookerjee et al. (Mookerjee et al., 2017, Mookerjee et al., 2015); these values are given in Supplementary Table 2.

\section{Flux analysis of digitonin-permeabilized cells by Seahorse XF96}

Cells were pre-incubated in a 96-well Seahorse culture plate in modified HBSS for ambient air with $5.5 \mathrm{mM}$ glucose and $0.1 \%\left(\mathrm{w} / \mathrm{v}\right.$ ) fatty acid-free BSA (Suppl. Table 1 ) for 1 hour at $37^{\circ} \mathrm{C}$ in ambient air. Subsequently, $50 \mu \mathrm{M}$ LRE1 or $0.1 \%$ DMSO (vehicle control) was added. After 10 minutes incubation, the media in all wells were replaced by MAS/BSA buffer (60 mM sucrose, $200 \mathrm{mM}$ mannitol, $10 \mathrm{mM}$ potassium phosphate buffer, $5 \mathrm{mM} \mathrm{MgCl}$, $1 \mathrm{mM}$ EGTA, $20 \mathrm{mM}$ HEPES and $0.4 \%$ fatty acid-free BSA, pH 7.1) as described by Salabei et al. (Salabei, Gibb et al., 2014) with the same substrates and inhibitors as in the previous incubation in HBSS. The plate was loaded onto the Seahorse XF96 Analyzer and the measurement started after a 10 minutes temperature equilibration. Cells were permeabilized with $25 \mu \mathrm{g} / \mathrm{mL}$ digitonin by injecting a concentrated mixture after baseline measurements. The injection mixture also contained 
831 substrates for mitochondrial respiration and 1 mM ADP (final concentration). The final

832 concentrations of substrates are $5 \mathrm{mM}$ succinate with $2.5 \mu \mathrm{M}$ rotenone, $2 \mathrm{mM}$ pyruvate with

$8331 \mathrm{mM}$ malate, and $4 \mathrm{mM}$ glutamate with $1 \mathrm{mM}$ malate. When substrates were omitted, oxygen

834 consumption decreased dramatically (data not shown). After about 15 minutes of ADP-driven

835 state 3 respiration, oligomycin A $(2.5 \mu \mathrm{M})$, FCCP $(2.5 \mu \mathrm{M})$, and antimycin A $(2.5 \mu \mathrm{M})$ plus

836 rotenone $(2.5 \mu \mathrm{M})$ were injected in order. The ADP-driven respiration rate was defined as the

837 difference between the first OCR measurement after the addition the mixture of digitonin,

838 ADP, and substrates and the last OCR measurement after the addition of oligomycin $A$. The

839 FCCP-driven respiration rate was defined as the difference between the first OCR

840 measurement after FCCP addition and the last OCR measurement after the addition of

841 oligomycin A.

\section{Statistics}

844 All results are presented as mean \pm standard deviation (SD). All $n$ number represent the 845 number of independent samples. Statistical significance was determined by two-tailed 846 Student's t-test, one-way analysis of variance (ANOVA) with Turkey's or Dunnett's multiple 847 comparison test, or two-way ANOVA with Sidak's multiple comparison test where applicable. 848 Statistical analysis was performed with GraphPad Prism 7 (GraphPad Software, La Jolla, CA) 849 with an $\alpha$ error of 0.05 . 


\section{Figure Legends}

852 Figure 1. Suppression of soluble adenylyl cyclase stably reprograms cell metabolism towards 853 a Warburg phenotype

854 (A-B) $\mathrm{H} 69$ human cholangiocytes were treated with different concentrations of SAC inhibitors $855 \mathrm{KH7}$ (A) and LRE1 (B). Medium glucose, L-lactate, and pyruvate were sampled hourly for 6 856 hours and enzymatically determined. (C) Immunoblot of SAC in short hairpin control (SHC) and 857 sAC-knockdown $\mathrm{H69}$ human cholangiocytes. (D) Glucose consumption, L-lactate secretion, 858 pyruvate secretion, and medium L-lactate-to-pyruvate ratio of SHC and SAC knockdown H69 859 human cholangiocytes. Results were normalized to protein contents. (E) HepG2 cells were 860 treated with SAC-specific inhibitors $\mathrm{KH7}$ and LRE1. Medium glucose, L-lactate and pyruvate 861 were sampled after 1, 2, 4, and 6 hours and enzymatically determined. Data presented as 862 mean $\pm S D$ ( $n=3$ for $A, B$ and $D ; n=2$ for $E$ ). Baseline value was the metabolite concentration 863 in the blank medium. Data are representative of at least two independent experiments.

Figure 2. Soluble adenylyl cyclase regulates the cytosolic $N A D H / N A D^{+}$redox state and aerobic glycolysis via complex I

(A) Ubiquitous monocarboxylate transporters (MCTs) equilibrate L-lactate and pyruvate across the plasma membrane. High lactate dehydrogenase activity in most cells brings the substrates (pyruvate and $\mathrm{NADH}$ ) and products (lactate and $\mathrm{NAD}^{+}$) to near equilibrium. Therefore, the extracellular lactate-to-pyruvate ratio reflects the cytosolic lactate-to-pyruvate ratio and the cytosolic NADH/NAD ratio. (B) In vivo calibration of the Peredox-mCherry NADH/NAD ${ }^{+}$redox sensor by media with different lactate-to-pyruvate ratio. (C-G) Cells were then pre-incubated

873 for 1 hour with $5.5 \mathrm{mM}$ glucose (C), no substrate (HBSS) (D, F, G) or $125 \mu \mathrm{M}$ octanoate (E). 874 Cells were refreshed again with the same medium and fluorescence ratio (R) of Peredox to 875 tandem-tagged mCherry was monitored. For calibration, cells were incubated with $5 \mathrm{mM}$ 876 lactate and $5 \mathrm{mM}$ pyruvate to obtain maximal $\left(R_{\max }\right)$ and minimal $\left(R_{\min }\right)$ ratios, respectively. 877 The results were normalized to $R_{\max }$ and $R_{\min }$. Arrows indicate additions of $0.1 \%$ DMSO (vehicle 878 control), $50 \mu \mathrm{M}$ LRE1, $5.5 \mathrm{mM}$ 2-deoxyglucose, or $0.5 \mathrm{mM}$ iodoacetate. Results are presented 879 as mean \pm SD $(n=3-4)$. (H-M) HepG2 cells were pre-treated with $0.1 \%$ DMSO or $50 \mu M$ LRE1 880 for 10 minutes in HBSS and then the medium was changed to MAS buffer. Oxygen consumption 881 rate (OCR) was monitored by Seahorse Flux Analyzer XF96. Cells were permeabilized by adding $88225 \mu \mathrm{g} / \mathrm{mL}$ digitonin together with $1 \mathrm{mM}$ ADP and substrates: (H-J) $2 \mathrm{mM}$ pyruvate and $1 \mathrm{mM}$ 
883

884

885

886

887

888

889

890

891

892

893

894

895

896

897

898

899

900

901

902

903

904

905

906

907

908

909

910

911

912

913

914

malate $(\mathrm{n}=4$ for DMSO and $\mathrm{n}=5$ for LRE1), and (K-M) $5 \mathrm{mM}$ succinate and $2.5 \mu \mathrm{M}$ rotenone ( $n=4$ for DMSO and $n=5$ for LRE1). Subsequently, $2.5 \mu M$ OA, $1 \mu M$ FCCP, and $2.5 \mu M$ antimycin $A(A A)+2.5 \mu \mathrm{M}$ rotenone (Rot) were injected as indicated. ADP-driven OCR (I and J) and FCCP-driven OCR ( $\mathrm{L}$ and $\mathrm{M})$ were derived from $(\mathrm{H})$ and $(\mathrm{K})$ as described in Methods. Data represent mean \pm SD. Statistical analysis: (I-J, L-M) Two-tailed unpaired Student's $t$-test. n.s., not significant, $* * \mathrm{P}<0.01, * * * \mathrm{P}<0.001$.

Figure 3. Soluble adenylyl cyclase is an acute switch for aerobic glycolysis that maintains energy homeostasis

The oxygen consumption rate (OCR) and extracellular acidification rate (ECAR) were analyzed by Seahorse Flux Analyzer XF96. (A-B) OCR and ECAR of confluent monolayers of H69 cholangiocytes were monitored in bicarbonate-free DMEM with $5.5 \mathrm{mM}$ glucose. (C-D) HepG2 cells were pre-incubated for 60 minutes with HBSS with $5.5 \mathrm{mM}$ glucose. OCR and ECAR were monitored. Arrows indicate the injection (in order) of $50 \mu \mathrm{M}$ LRE1 or $0.1 \%$ DMSO, oligomycin A (OA), carbonyl cyanide-p-trifluoromethoxyphenyl hydrazone (FCCP), and antimycin A (AA) and rotenone (Rot). Data represent mean \pm SD of (A-B) $n=5$ and $5,(C-D) n=10$ and 9 for DMSO and LRE1, respectively. (E-L) HepG2 cells were preincubated for 1 hour in HBSS for ambient air without substrates (E-F), with $125 \mu \mathrm{M}$ octanoate (G-H), with $5.5 \mathrm{mM}$ glucose and $125 \mu \mathrm{M}$ octanoate (I-J), and with $125 \mu \mathrm{M}$ octanoate $\pm 5.5 \mathrm{mM}$ 2-deoxyglucose (2-DG) (K-L). Oxygen consumption rate (OCR) and extracellular acidification rate (ECAR) were measured with Seahorse Flux Analyzer XF96. Arrows indicate the injection (in order) of 0.1\% DMSO (vehicle control) or $50 \mu \mathrm{M}$ LRE1, oligomycin A (OA), carbonyl cyanide-ptrifluoromethoxyphenyl hydrazone (FCCP), and antimycin A (AA) and rotenone (Rot). Data are presented as mean \pm SD of $(E-F) n=10$ and $8,(G-H) n=10$ and $11,(I-J) n=10$ and 8 for DMSO and LRE1 respectively. Data in (K-L) represents mean \pm SD of $n=8$ and 6 for octanoate + LRE1 and octanoate/2-DG + LRE1 respectively. All data are representative of at least two independent experiments. Statistical analysis: (A-B) two-tailed unpaired Student's $t$-test. $* \mathrm{P}<0.05$.

\section{Figure 4. Inhibition of soluble adenylyl cyclase promotes glycogenolysis}

(A) HepG2 cells were preincubated with $5.5 \mathrm{mM}$ glucose for 1 hour. After harvesting baseline samples $(n=3)$, cells were refreshed with medium $\pm 5.5 \mathrm{mM}$ glucose $\pm 50 \mu \mathrm{M}$ LRE1 for another 
915 hour and then harvested for glycogen determination. Data represent mean $\pm S D(n=3)$. (B) $916 \mathrm{H} 69$ cholangiocytes were treated as in (A) and glycogen content of cells were determined. Data 917 presents mean \pm SD of $n=8$ for baseline and $n=2$ for \pm 5.5 mM glucose $\pm 50 \mu M$ LRE1. (C-D) 918 HepG2 cells were pre-incubated with $125 \mu \mathrm{M}$ octanoate in the presence or absence of CP91991149 for 1 hour. Oxygen consumption rate (OCR) and extracellular acidification rate (ECAR) 920 were measured by Seahorse Flux Analyzer XF96. Arrows indicate the injection (in order) of 50 $921 \mu \mathrm{M}$ LRE1, oligomycin A (OA), carbonyl cyanide-p-trifluoromethoxyphenyl hydrazone (FCCP), 922 and antimycin $A(A A)$ and rotenone (Rot). Data are presented as mean $\pm S D$ of $n=5$ and 5 for 923 octanoate/DMSO and octanoate/CP-91149, respectively. (E-F) HepG2 cells were pre924 incubated in HBSS without substrates in the presence or absence of CP-91149 for 1 hour. OCR 925 and ECAR were measured as in (C) and (D). Data are presented as mean \pm SD of $n=5$ and 6 926 for no substrate/DMSO and no substrate/CP-91149, respectively. (G-H) Fluorescence ratio (R) 927 of Peredox to tandem-tagged mCherry was monitored in non-starved or 4-hour starved 928 HepG2 cells expressing Peredox-mCherry biosensor. Arrows indicate the addition of $0.1 \%$ 929 DMSO (vehicle control) or $50 \mu \mathrm{M}$ LRE1. For calibration, cells were incubated with $5 \mathrm{mM}$ lactate 930 and $5 \mathrm{mM}$ pyruvate to obtain maximal $\left(R_{\max }\right)$ and minimal $\left(R_{\min }\right)$ ratios, respectively. The 931 results were normalized to $R_{\max }$ and $R_{\min }$. (I-J) HepG2 cells were pre-incubated in glucose-free 932 HBSS with $125 \mu \mathrm{M}$ octanoate for 1 hour and then treated with $0.1 \%$ or $0.2 \%$ DMSO (vehicle control), $50 \mu \mathrm{M}$ or $100 \mu \mathrm{M}$ LRE1, and $15 \mathrm{nM}$ or $30 \mathrm{nM}$ rotenone for 15 minutes. Glycogen level

934 (I) and secreted lactate (J) were assayed. Data are presented as mean \pm SD ( $\mathrm{n}=3$ for $0.1 \%$ DMSO condition of glycogen, $n=4$ for the rest of the conditions) (K) HepG2 cells were preincubated with $125 \mu \mathrm{M}$ octanoate 1 hour. Extracellular acidification rate (ECAR) was monitored by Seahorse Flux Analyzer XF96. The arrow indicates addition of 0.1\% DMSO (vehicle control, $n=7), 50 \mu M$ LRE1 $(n=8)$, and $15 n M$ rotenone $(n=8)$. Data are presented as mean \pm SD. Statistical analysis: (A-B) Two-way ANOVA with Tukey's multiple comparisons test. (I-J) One-way ANOVA with Dunnett's multiple comparisons test (against 0.1\% DMSO). n.s., not significant, ${ }^{*} \mathrm{P}<0.05, * * \mathrm{P}<0.01, * * * \mathrm{P}<0.001$. LRE1 $(n=3)$, and $1 \mu$ M forskolin $(n=3)$ for 60 minutes. Glycogen content at baseline $(n=6)$ and 60 minutes (A) and medium L-lactate at 15, 30, 60 minutes (B) were assayed. Data represent mean \pm SD. (C) HepG2 cells were pre-incubated for 1 hour in HBSS for ambient air 
with $125 \mu \mathrm{M}$ octanoate or $0.1 \%$ BSA. Extracellular acidification rate (ECAR) was measured with Seahorse Flux Analyzer XF96. After baseline measurement, 0.1\% DMSO (vehicle control, $n=$ 6), $50 \mu \mathrm{M}(\mathrm{R})$-CE3F4 ( $\mathrm{n}=6), 10 \mu \mathrm{M} \mathrm{ESI-05}(\mathrm{n}=4)$, or $10 \mu \mathrm{M} \mathrm{H89}(\mathrm{n}=5)$ were injected, followed by oligomycin A (OA), carbonyl cyanide-p-trifluoromethoxyphenyl hydrazone (FCCP), and antimycin $A(A A)$ and rotenone (Rot). Data are presented as mean \pm SD. (D) Same as (C) but in HBSS with $0.1 \%$ BSA. Data are presented as mean \pm SD for $0.1 \%$ DMSO (vehicle control, $n=6$ ), $50 \mu \mathrm{M}(\mathrm{R})$-CE3F4 ( $n=6), 10 \mu \mathrm{M}$ ESI-05 ( $n=5)$, or $10 \mu \mathrm{M} \mathrm{H89}(\mathrm{n}=5)$. (E-F) HepG2 cells (E) and H69 cholangiocytes (F) were pre-incubated in $5.5 \mathrm{mM}$ glucose for 1 hour and acutely treated with $0.1 \%$ DMSO (vehicle control), $50 \mu \mathrm{M}$ (R)-CE3F4, $10 \mu \mathrm{M}$ ESI-05, $10 \mu \mathrm{M}$ H89, or $100 \mu \mathrm{M}$ dibutyryl-cAMP (db-cAMP) in glucose-free medium. Glycogen content was determined and normalized to total protein. Data are presented as mean $\pm S D(n=2)$. (G-H) HepG2 cells $(G)$ and $\mathrm{H} 69$ cholangiocytes $(\mathrm{H})$ were pre-incubated in $5.5 \mathrm{mM}$ glucose for 1 hour and acutely treated with 0.1\% DMSO (vehicle control), $50 \mu \mathrm{M}$ (R)-CE3F4, $10 \mu \mathrm{M} \mathrm{ESI-05,} 10 \mu \mathrm{M} \mathrm{H89,} \mathrm{or} 100$ $\mu \mathrm{M} \mathrm{db}$-cAMP in medium containing $5.5 \mathrm{mM}$ glucose. Glycogen content was determined and normalized to total protein. Data are presented as mean $\pm S D(n=2)$. (I) H69 cells were treated with $50 \mu \mathrm{M}$ LRE1 or $1 \mu \mathrm{M}$ forskolin for 5,15 , and 30 minutes. Phosphorylation of Ser 15 of liver form glycogen phosphorylase (PYGL) was examined by immunoblotting. (J) H69 cells were treated with $50 \mu \mathrm{M}(\mathrm{R})$-CE3F4 or $1 \mu \mathrm{M}$ forskolin for 5, 15, and 30 minutes. Phosphorylation of Ser 15 of liver form glycogen phosphorylase (PYGL) was examined by immunoblotting. Statistical analysis: (A, E-H) One-way ANOVA with Dunnett's multiple comparisons test (against DMSO group). n.s., not significant, ${ }^{*} \mathrm{P}<0.01,{ }^{* * *} \mathrm{P}<0.001$.

Figure 6. Working model of the acute regulation of aerobic glycolysis and glycogenolysis by soluble adenylyl cyclase

972 The activity of soluble adenylyl cyclase (SAC) is dependent on ATP and stimulated by $\mathrm{Ca}^{2+}$ and $973 \mathrm{HCO}_{3}-$ On the one hand, SAC regulates complex I activity; on the other hand, SAC-CAMP-Epac1 974 signaling suppresses glycogenolysis by reducing Ser15 phosphorylation of glycogen 975 phosphorylase while the established tmAC-cAMP-PKA signaling activates glycogenolysis by 976 increasing Ser15 phosphorylation of glycogen phosphorylase. When ATP level falls, decreased 977 SAC activity induces a Warburg-like metabolic phenotype to maintain energy homeostasis, 978 which is characterized by suppression of oxidative phosphorylation, promotion of glycolysis, 979 and induction of glycogenolysis. 


\section{Supplementary Figure Legends}

981 Figure S1. Suppression of SAC activity promotes glycolysis (related to Figure 1).

982 (A-B) H69 cholangiocytes were treated with pan-phosphodiesterase inhibitor IBMX in the 983 presence or absence of SAC inhibitor KH7 (A) or LRE1 (B) for 5 minutes. The accumulated cAMP 984 was assayed by ELISA. Data represent mean $\pm S D(n=3)$. (C-F) primary mouse hepatocytes $(C)$, 985 human colorectal carcinoma Caco-2 (D), Abelson murine leukemia virus-transformed 986 macrophage Raw264.7 (E), and murine melanoma B16F10 (F) were treated with $50 \mu \mathrm{M}$ LRE1 987 or $0.1 \%$ DMSO. Medium glucose, L-lactate, and pyruvate were sampled hourly for 4 hours and 988 enzymatically determined. Data represent mean \pm SD $(n=3)$. Statistical analysis: (A-B) Two989 tailed unpaired Student's $t$-test. ${ }^{*} P<0.05,{ }^{* * *} P<0.001$.

990

991 Figure S2. Soluble adenylyl cyclase regulates the cytosolic NADH/NAD+ redox state and 992 aerobic glycolysis via complex I (related to Figure 2).

993 (A-B) Intracellular lactate-to-pyruvate ratios in $\mathrm{H} 69$ cholangiocytes (A) and HepG2 cells (B) 994 treated with $0.1 \%$ DMSO, $25 \mu \mathrm{M}$ and $50 \mu \mathrm{M}$ LRE1 for 6 hours. Data represent mean \pm SD $(n=$ 9953 for $A, n=2$ for $B$ ). (C-E) HepG2 cells were pre-treated with $0.1 \%$ DMSO or $50 \mu M$ LRE1 for 10 996 minutes in HBSS and then the medium was changed to MAS buffer. Oxygen consumption rate 997 (OCR) was monitored by Seahorse Flux Analyzer XF96. Cells were permeabilized by adding 25 $998 \mu \mathrm{g} / \mathrm{mL}$ digitonin together with $1 \mathrm{mM}$ ADP, $4 \mathrm{mM}$ glutamate, and $1 \mathrm{mM}$ malate $(\mathrm{n}=5$ and 4 for 999 DMSO and LRE1, respectively). Subsequently, $2.5 \mu \mathrm{M} \mathrm{OA}, 1 \mu \mathrm{M} \mathrm{FCCP}$, and $2.5 \mu \mathrm{M}$ antimycin $\mathrm{A}$ 1000 (AA) plus $2.5 \mu \mathrm{M}$ rotenone (Rot) were injected as indicated. ADP-driven OCR (D) and FCCP1001 driven OCR (E) were derived from (C) as described in Methods. Data represent mean \pm SD. (F$1002 \mathrm{H})$ HepG2 cells were treated with 0.1\% DMSO (vehicle control), $10 \mathrm{nM}$ rotenone, $30 \mathrm{nM}$ 1003 rotenone, and $50 \mu \mathrm{M}$ LRE1 in medium containing $5.5 \mathrm{mM}$ glucose for 1 hour. Medium L-lactate $1004(F)$, pyruvate $(G)$, and their ratio $(H)$. Data represent mean \pm SD $(n=2)$. Statistical analysis: (A1005 B) One-way ANOVA with Tukey's multiple comparisons test. (D-E) Two-tailed unpaired 1006 Student's $t$-test. (F-H) One-way ANOVA with Tukey's multiple comparisons test. n.s., not 1007 significant; ${ }^{*} \mathrm{P}<0.05, * * \mathrm{P}<0.01, * * * \mathrm{P}<0.001$.

1008

Figure S3. Soluble adenylyl cyclase is an acute switch for aerobic glycolysis that maintains 
1011 (A) H69 cholangiocytes were pre-treated with SAC inhibitor (50 $\mu \mathrm{M} \mathrm{KH7)} \mathrm{for} 10$ minutes and 1012 then pan-phosphodiesterase inhibitor IBMX was added to initiate cAMP accumulation in the 1013 presence or absence of in $5 \% \mathrm{CO}_{2}$ incubator or in ambient air. After 6 minutes, the 1014 accumulated cAMP was assayed by competitive ELISA. Data is normalized to DMSO $5 \% \mathrm{CO}_{2}$ 1015 group and represents mean \pm SD $(n=2)$. (B-G) ATP production rates by oxidative 1016 phosphorylation $\left(J_{\text {ATP_OxPhos }}\right)(B)$, tricarboxylic cycle $\left(J_{\text {ATP_TCA }}\right)(C)$, mitochondria $\left(J_{\text {ATP_Mitochondria) }}\right)(D)$, 1017 glycolysis ( $\left.J_{\text {ATP_Glycolysis }}\right)(E)$, and total ATP production rate $\left(J_{\text {ATP_Total }}\right)(F)$ were derived from OCR 1018 and ECAR measurements in Figure 2C and 2D as described in Methods. (G) HepG2 cells were 1019 treated with $0.1 \%$ DMSO (vehicle control) or $50 \mu \mathrm{M}$ LRE1 for 10 and 90 minutes. AMP, ADP, 1020 and ATP were measured for the calculation of the adenylate energy charge. Data represent 1021 mean \pm SD $(n=3)$. $(H-L)$ ATP production rates by oxidative phosphorylation $\left(J_{\text {ATP_oxphos }}\right)(H)$, 1022 tricarboxylic cycle $\left(J_{\text {ATP_TCA }}\right)(I)$, mitochondria $\left(J_{\text {ATP_Mitochondria }}\right)(J)$, glycolysis $\left(J_{\text {ATP_Glycolysis }}\right)(K)$, and 1023 Total ATP production rate $\left(J_{\text {ATP_Total }}\right)(L)$ were derived from OCR and ECAR measurements in 1024 Figure $3 \mathrm{G}$ and $3 \mathrm{H}$ as described in Methods. is defined as the sum of $J_{\text {ATP_Glycolysis }}$ and $J_{\text {ATP_Mitochondria. }}(\mathrm{M})$ HepG2 cells were treated with $0.1 \%$ DMSO (vehicle control) or $50 \mu \mathrm{M}$ LRE1 for 10 and 90 minutes. AMP, ADP, and ATP were measured for the calculation of the adenylate energy charge. Data represent mean $\pm S D(n=3)$. Statistical analysis: $(A)$ Two-way ANOVA with Sidak's multiple comparisons test. ${ }^{*} \mathrm{P}<0.05$. (related to Figure 4).

1032 (A) HepG2 cells acutely starved in the presence of $0.1 \%$ DMSO, $50 \mu M$ LRE1, or $50 \mu M$ LRE1 + $100 \mu \mathrm{M}$ CP-91149. Glycogen contents were determined and normalized to protein content. Data are presented as mean $\pm S D(n=3)$. (B) Primary mouse hepatocytes were acutely starved in the presence or absence of $50 \mu \mathrm{M}$ LRE1 and $100 \mu \mathrm{M}$ CP-91149 for 1.5 hours. Glycogen contents were determined and normalized to protein content. Data represent mean $\pm S D(n=$ 3). (C) Primary mouse hepatocytes were incubated with $50 \mu \mathrm{M}$ LRE1 or vehicle control for 4 hours. Glycogen contents were determined and normalized to protein content. Data represent mean $\pm S D(n=3)$. (D) HepG2 cells were incubated in medium with 0,1 , and $5 \mathrm{mM}$ glucose in the presence or absence $50 \mu \mathrm{M}$ LRE1 for 3 hours. Glycogen contents were determined and normalized to protein content. Data represent mean $\pm S D(n=3)$. (E) HepG2 cells were preincubated for 60 minutes with HBSS with $5.5 \mathrm{mM}$ glucose. Oxygen consumption rate was 
1043

1044

1045

1046

1047

1048

1049

1050

1051

1052

1053

1054

1055

1056

1057

1058

1059

1060

1061

1062

1063

1064

1065

1066

1067

1068

1069

monitored. Arrows indicate the injection (in order) of 0.1\% DMSO $(n=8), 50 \mu M \operatorname{LRE1}(n=4)$, $10 n M$ rotenone $(n=4)$, or $15 n M$ rotenone $(n=5)$, oligomycin A $(O A)$, carbonyl cyanide- $p$ trifluoromethoxyphenyl hydrazone (FCCP), and antimycin A (AA) and rotenone (Rot). The coupled respiration rate $(F)$ and the FCCP-uncoupled respiration rate $(G)$ were derived from (E) as described in Materials and Methods. \# denotes values below the detection limit. Statistical analysis: (A-B) Two-way ANOVA with Sidak's multiple comparisons test. (C) Twotailed unpaired Student's $t$-test. (D) Two-way ANOVA with Tukey's multiple comparisons test. (F-G) One-way ANOVA with Dunnett's multiple comparisons test (against DMSO group). n.s., not significant; ${ }^{*} P<0.05,{ }^{*} P<0.01, * * * P<0.001$.

Supplementary Figure 5. SAC-Epac1 and tmAC-PKA signaling have opposite effects on glycogen homeostasis (related to Figure 5).

(A-C) HepG2 cells were acutely starved of glucose and treated with 0.1\% DMSO (vehicle control), $50 \mu \mathrm{M}$ (R)-CE3F4, $10 \mu \mathrm{M}$ ESI-05, $10 \mu \mathrm{M}$ H89, $100 \mu \mathrm{M}$ db-cAMP. Medium L-lactate and pyruvate were assayed at indicated time points. Data represent mean $\pm S D(n=2)$. (D-F) H69 cells were acutely starved of glucose and treated with 0.1\% DMSO (vehicle control), 50 $\mu \mathrm{M}(\mathrm{R})$-CE3F4, $10 \mu \mathrm{M}$ ESI-05, $10 \mu \mathrm{M}$ H89, $100 \mu \mathrm{M}$ db-cAMP. Medium L-lactate and pyruvate were assayed at indicated time points. Data represent mean $\pm S D(n=2)$. (G-I) HeLa cells (G), Caco-2 cells (H), HEK293T cells (I) were pre-incubated 15 minutes in glucose-free DMEM and then refreshed with glucose-free DMEM with $0.1 \%$ DMSO (vehicle control), $50 \mu M$ LRE1, and $50 \mu \mathrm{M}(\mathrm{R})$-CE3F4 for 45 minutes and then the glycogen content was determined. Data represent mean $\pm S D(n=4)$. Statistical analysis: ( $C$ and F) One-way ANOVA with Dunnett's multiple comparisons test (against DMSO group). n.s., not significant; ${ }^{*} \mathrm{P}<0.05,{ }^{* * *} \mathrm{P}<0.001$. (G-I) One-way ANOVA with Dunnett's multiple comparisons test (against DMSO group). n.s., not significant; ${ }^{*} \mathrm{P}<0.05,{ }^{* *} \mathrm{P}<0.01,{ }^{* * *} \mathrm{P}<0.001$. 


\section{Figure 1}

A
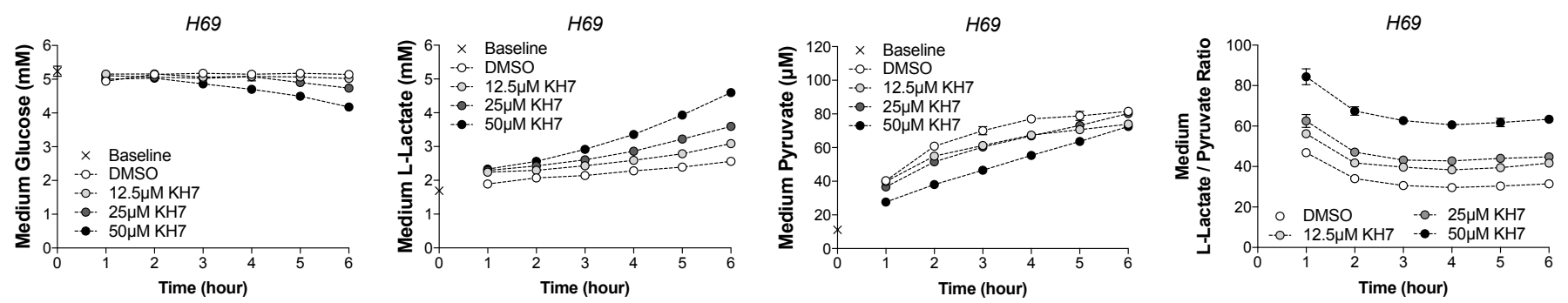

B
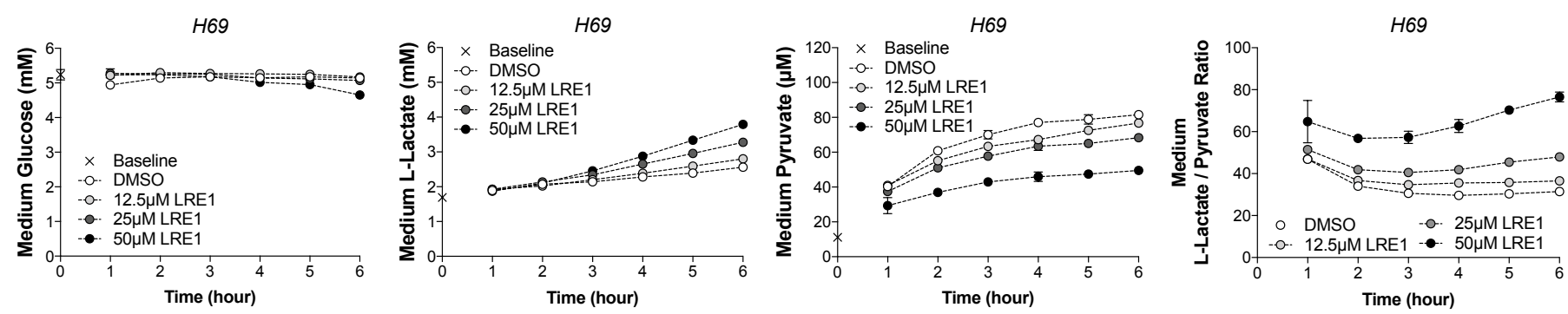

C

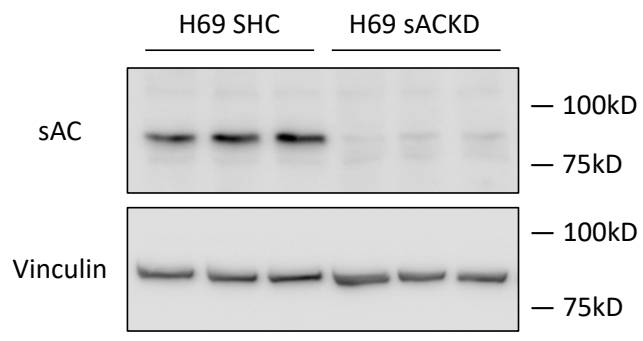

D
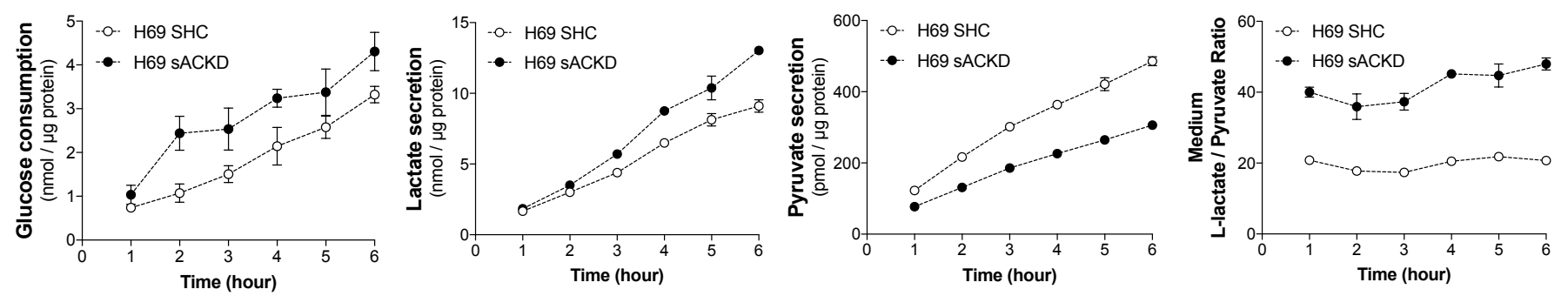

E
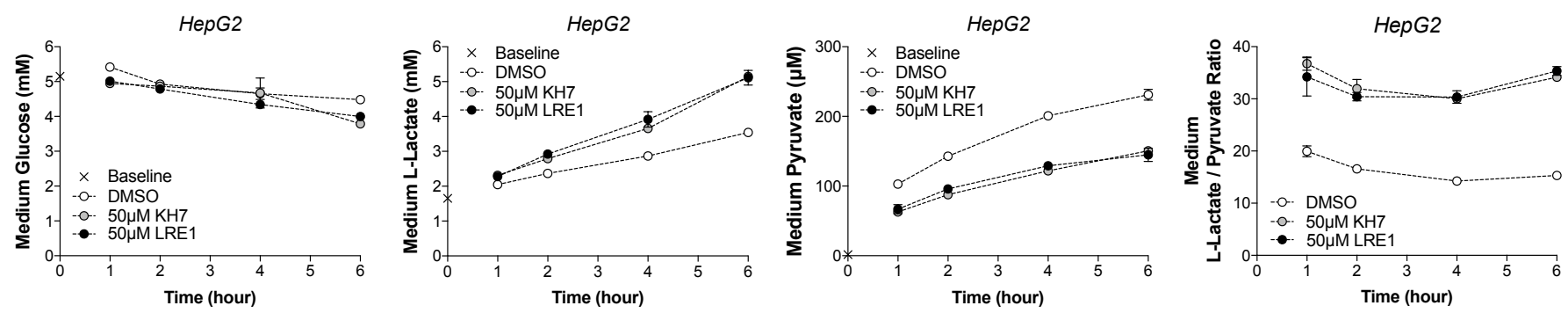
A

B

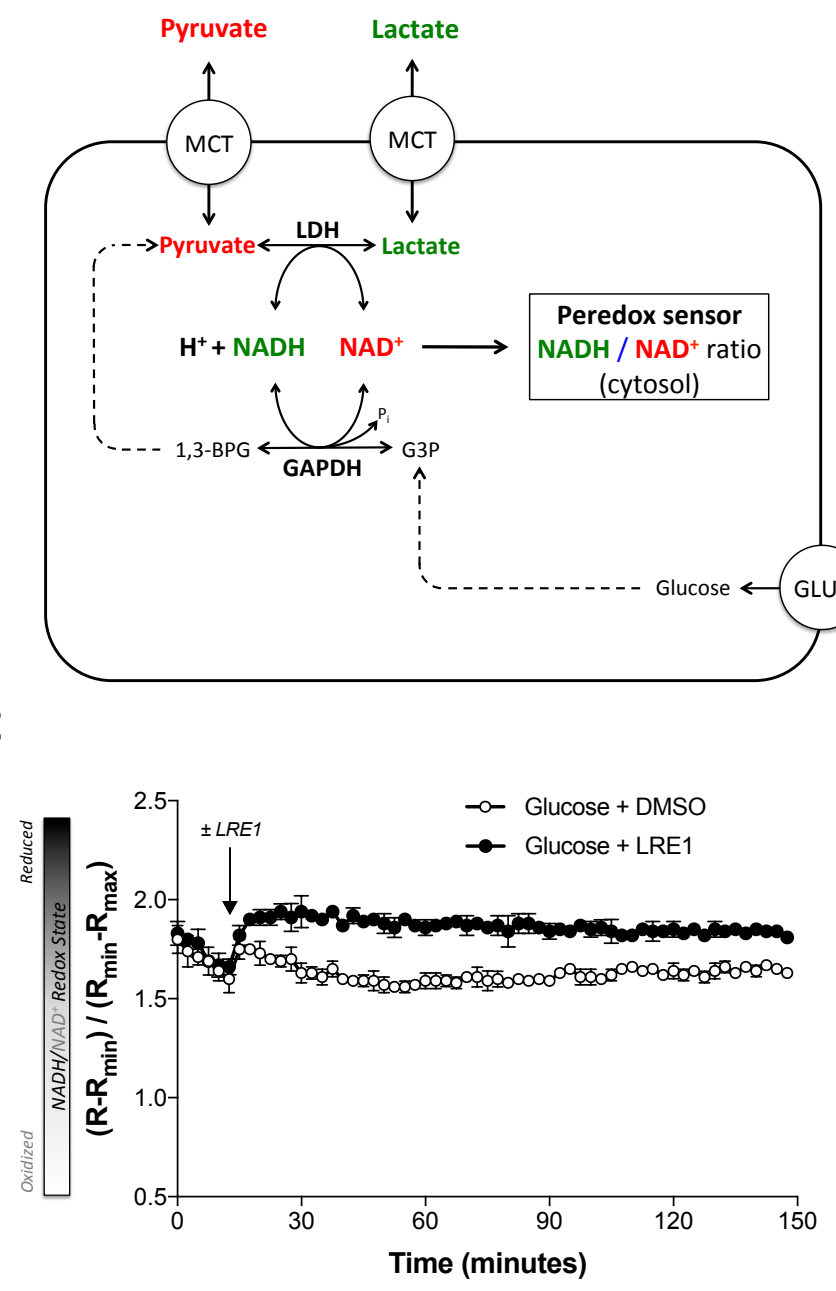

E

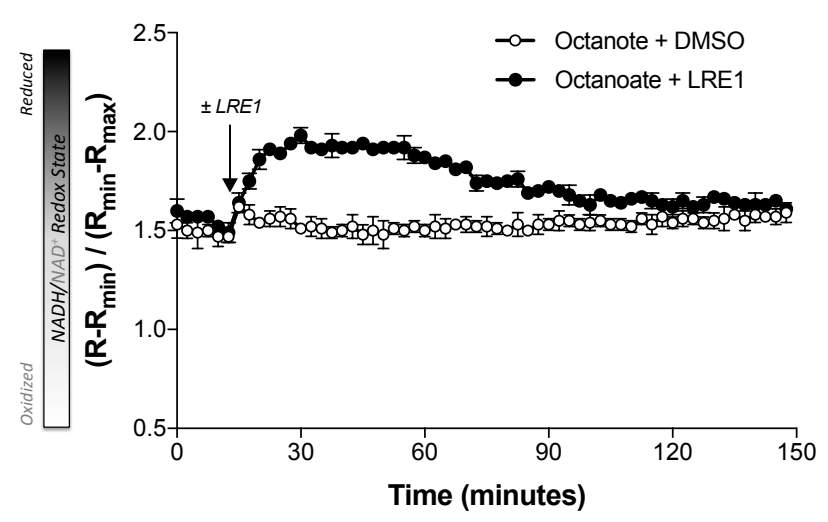

G

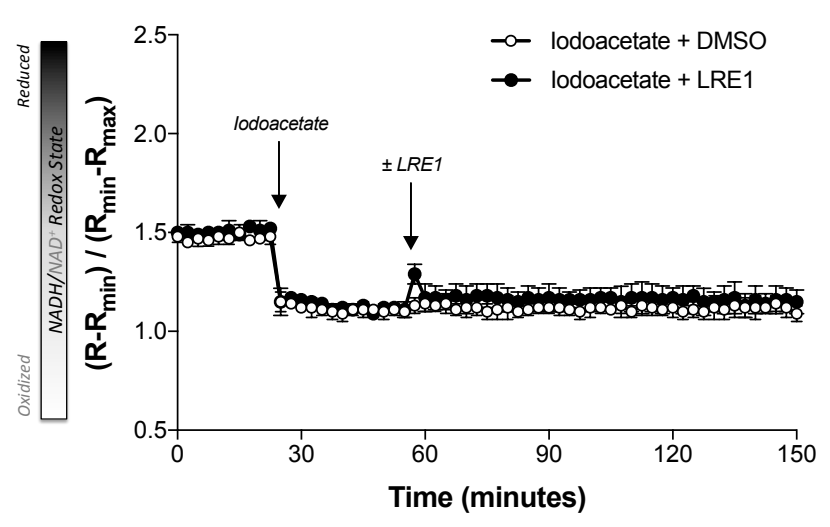

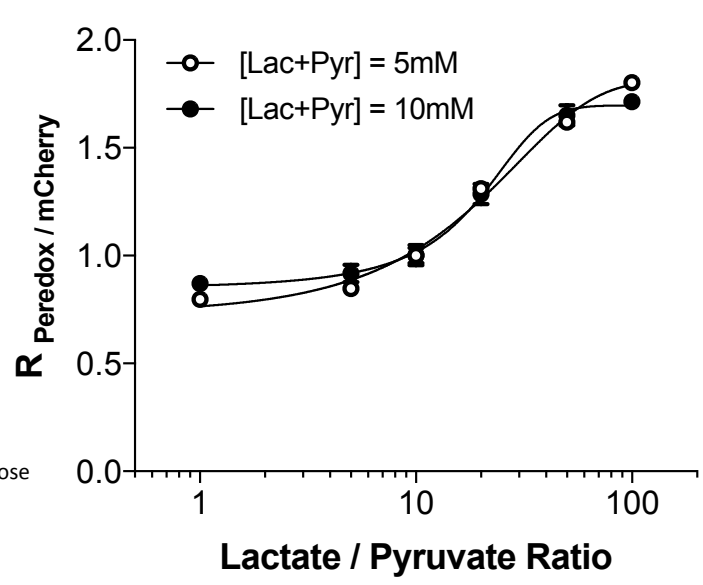

D

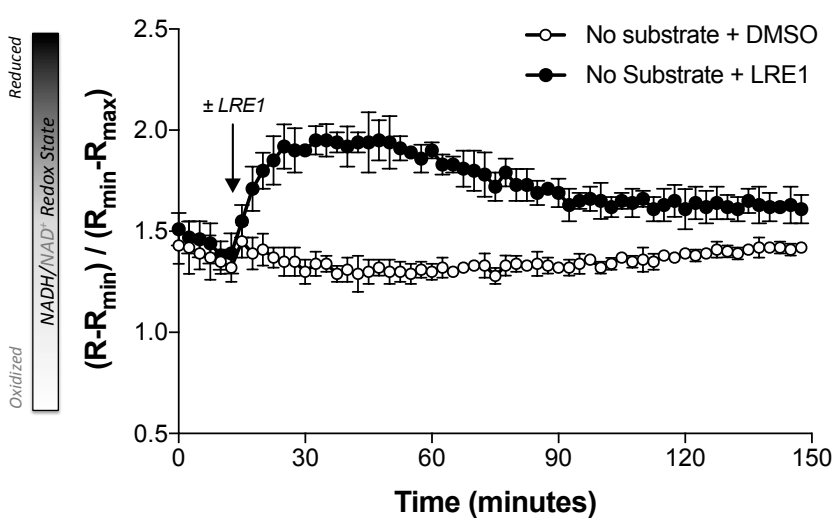

F

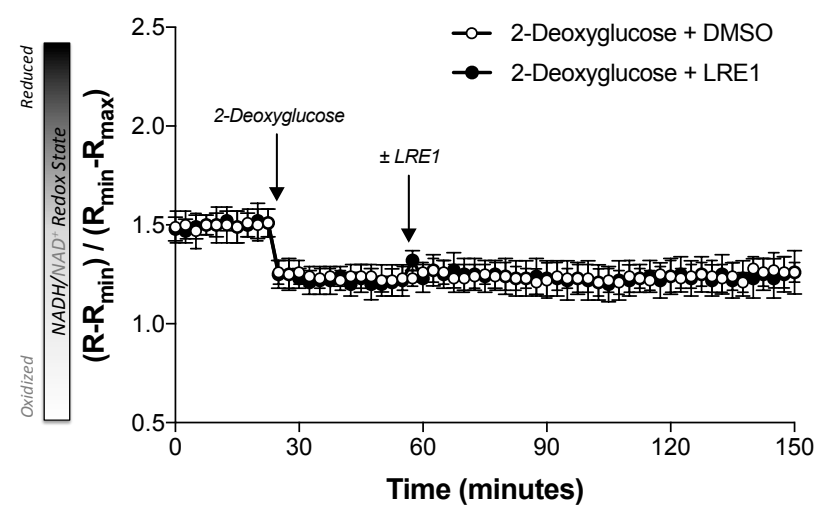


Figure 2 (continued)

H

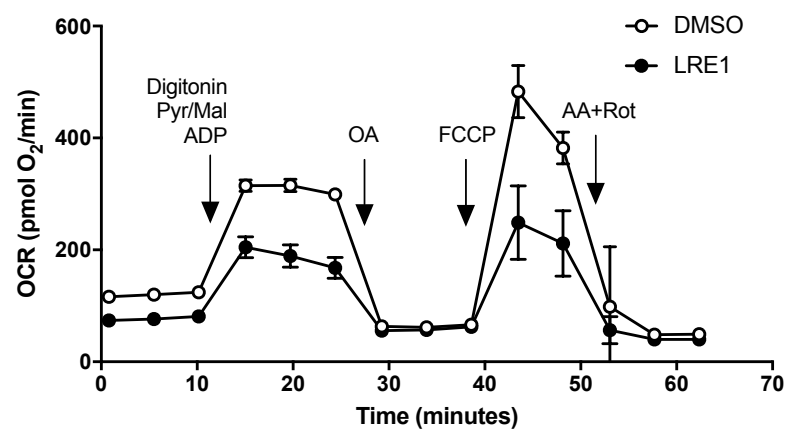

K

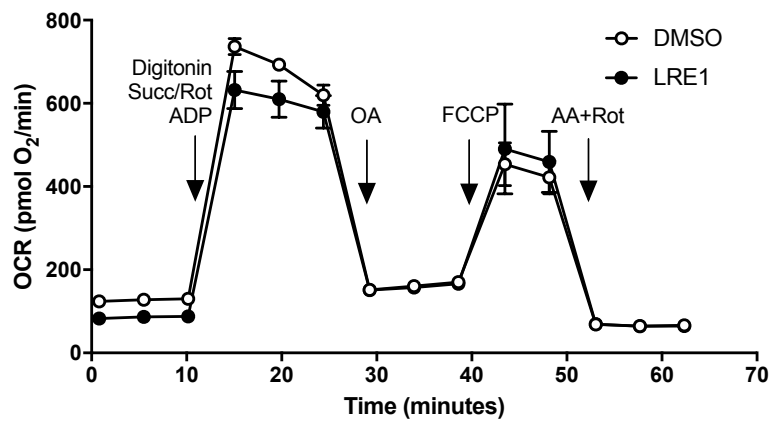

I

J

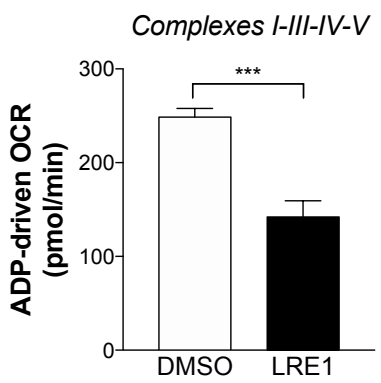

L

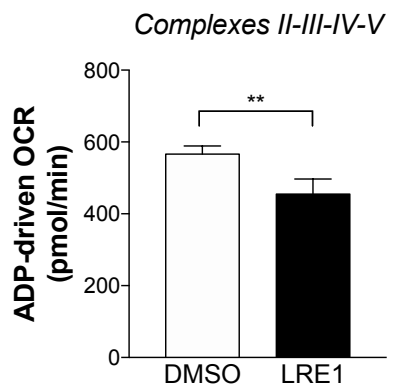

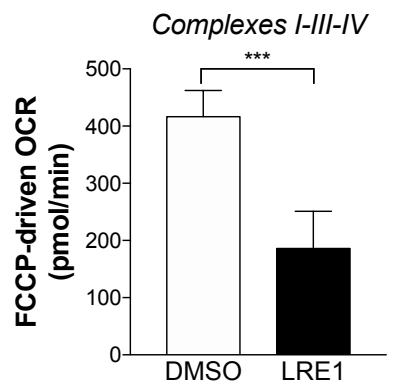

M

Complexes II-III-IV

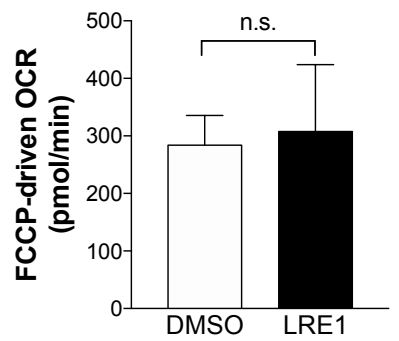




\section{Figure 3}

A

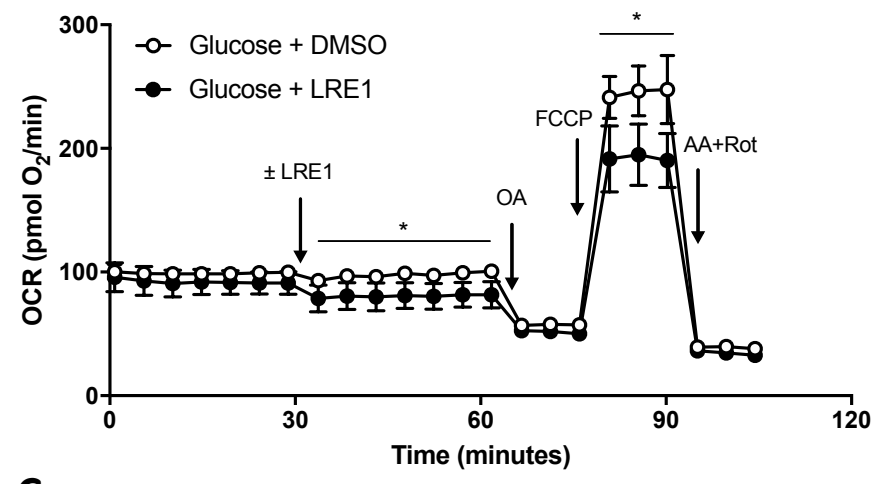

C

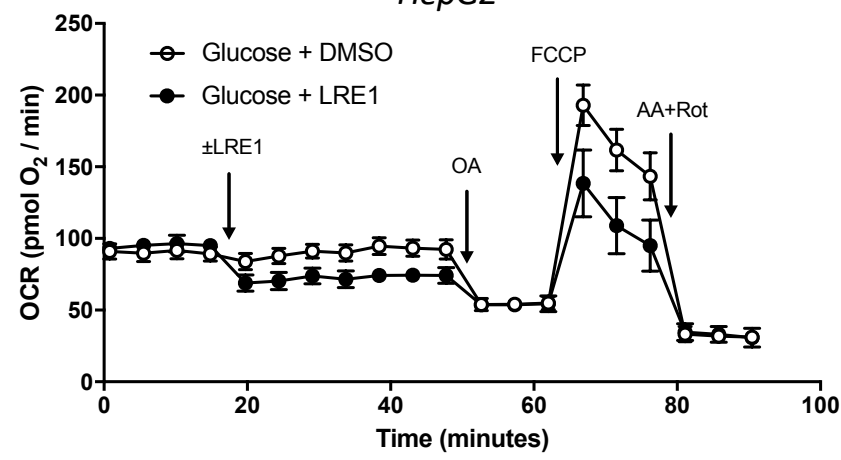

E

HepG2

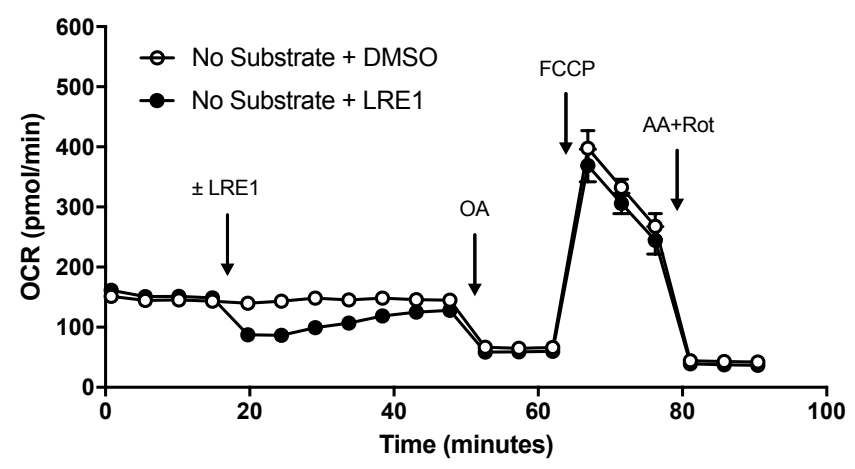

G

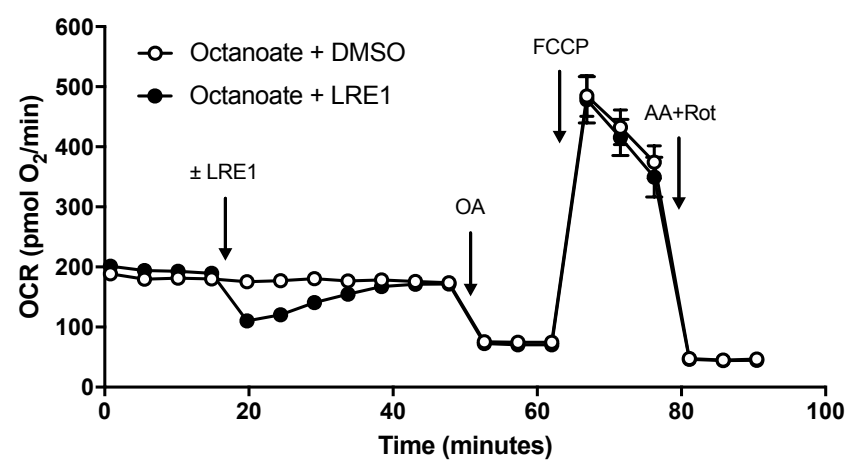

B

H69

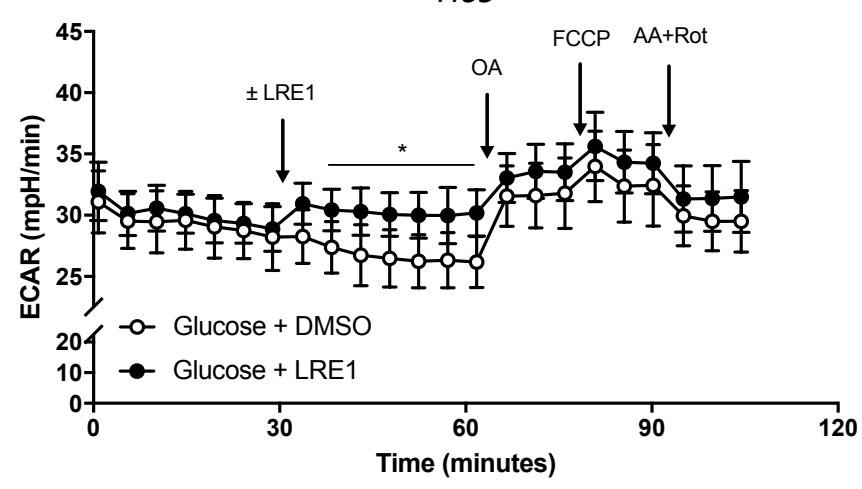

D

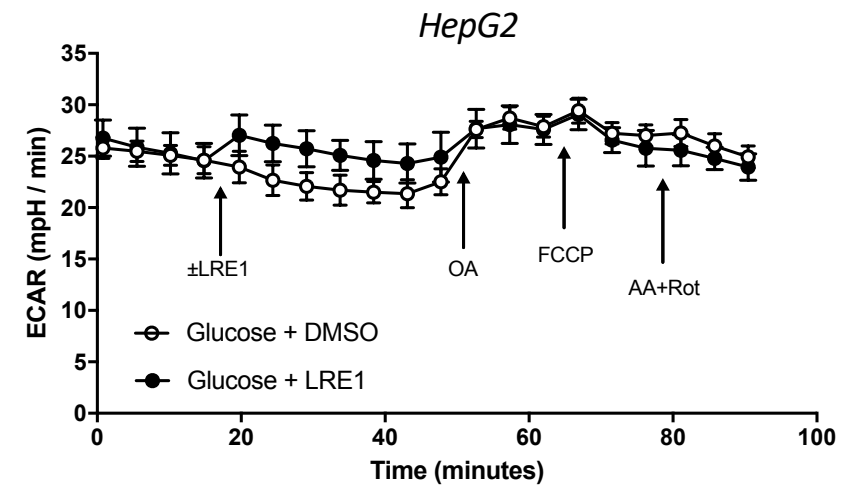

F

HepG2

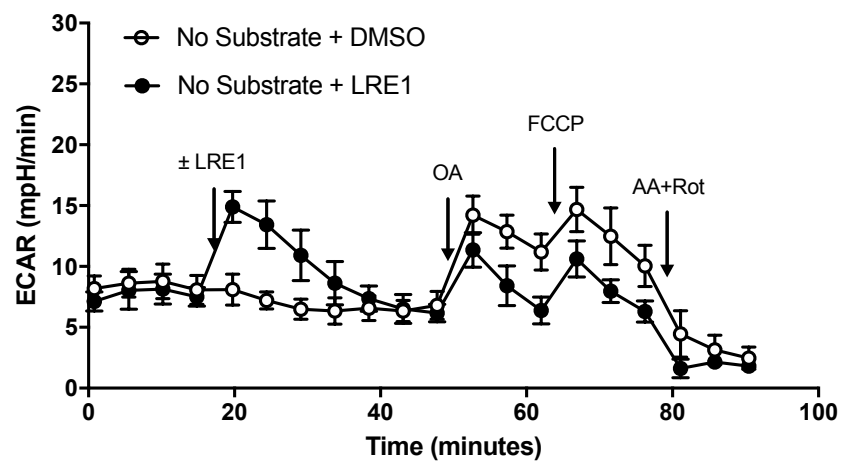

H

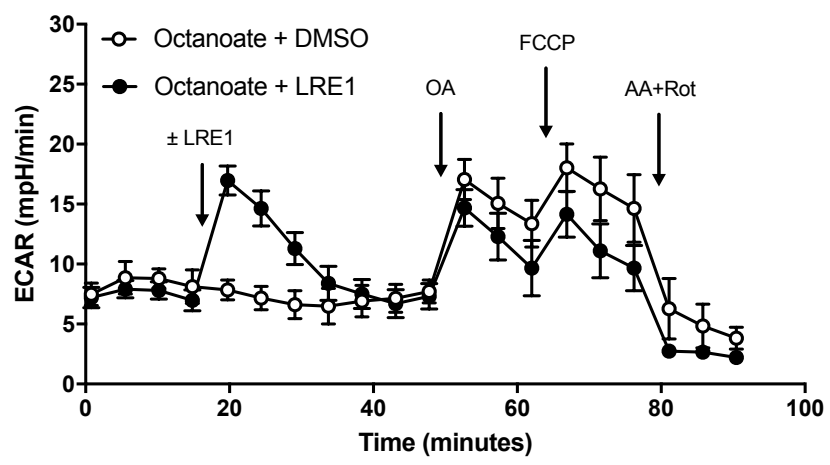


I

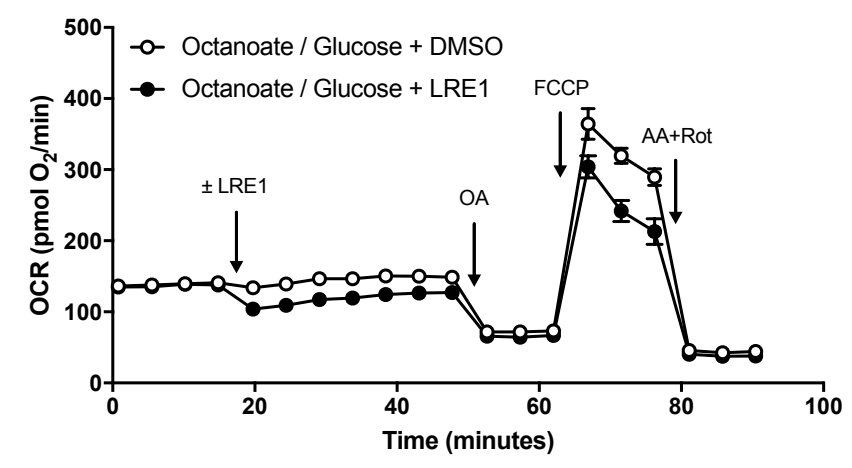

K

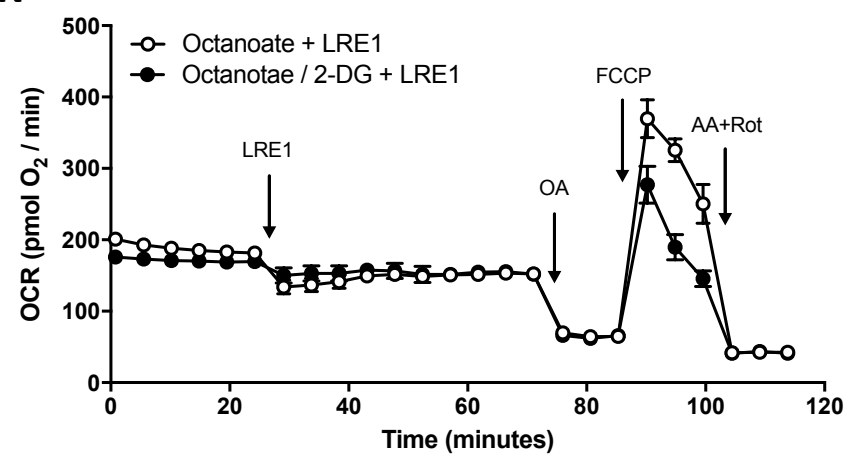

J

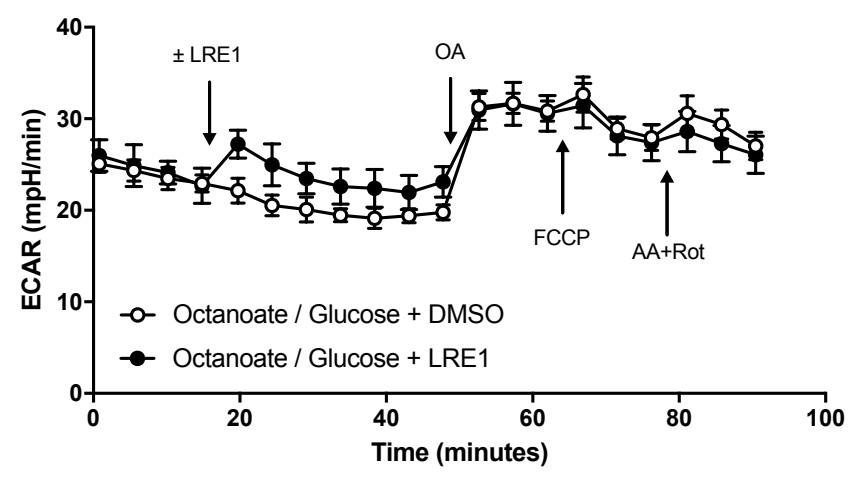

L

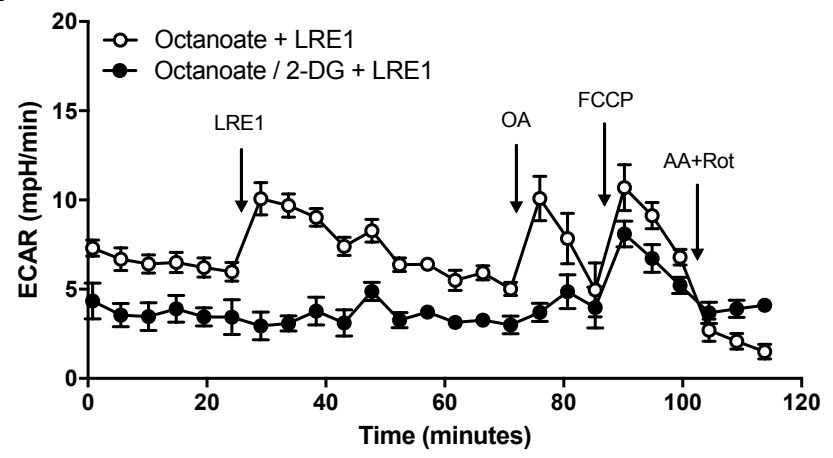


A

HepG2
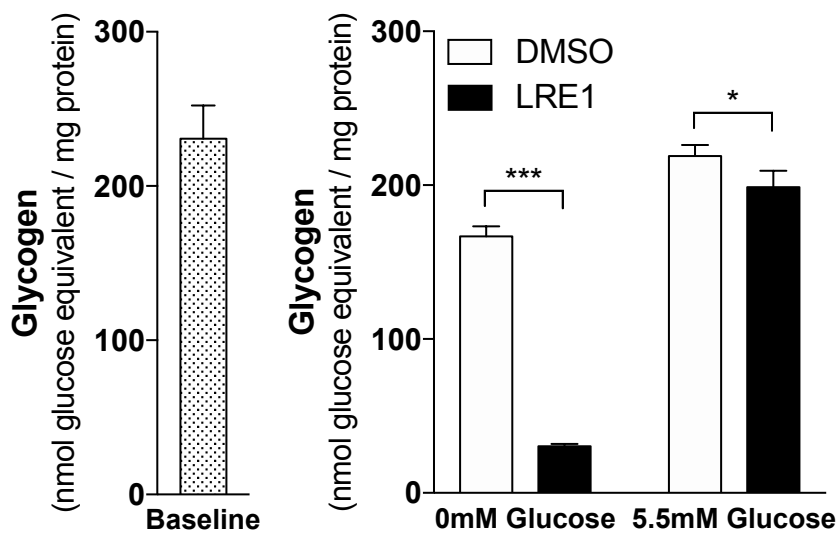

1 hr incubation
B

H69

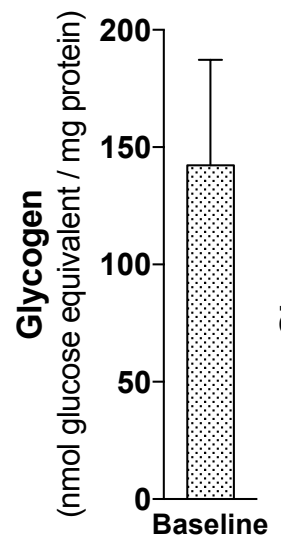

D

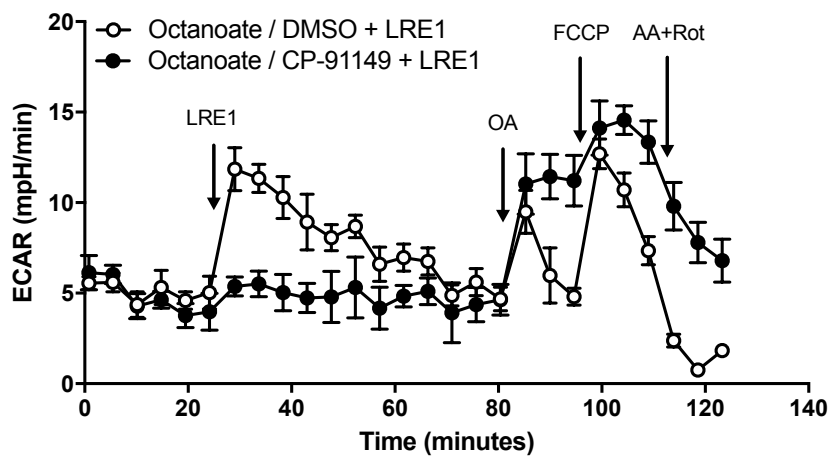

$\mathbf{F}$

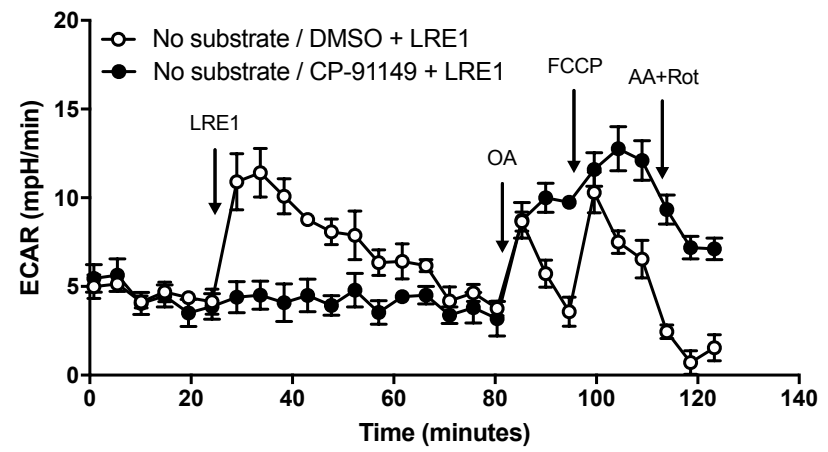

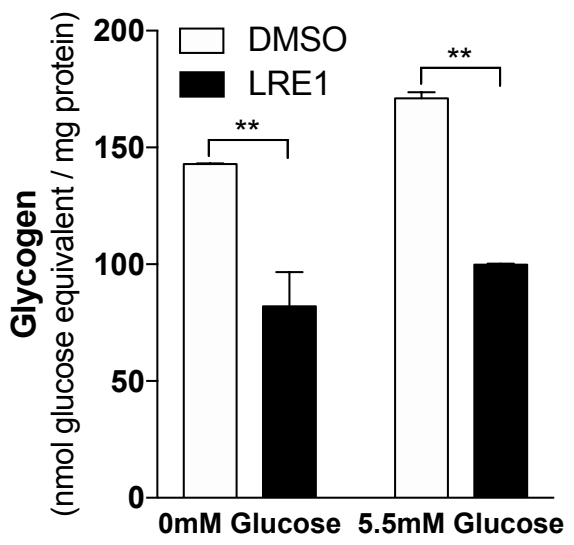

$1 \mathrm{hr}$ incubation

C

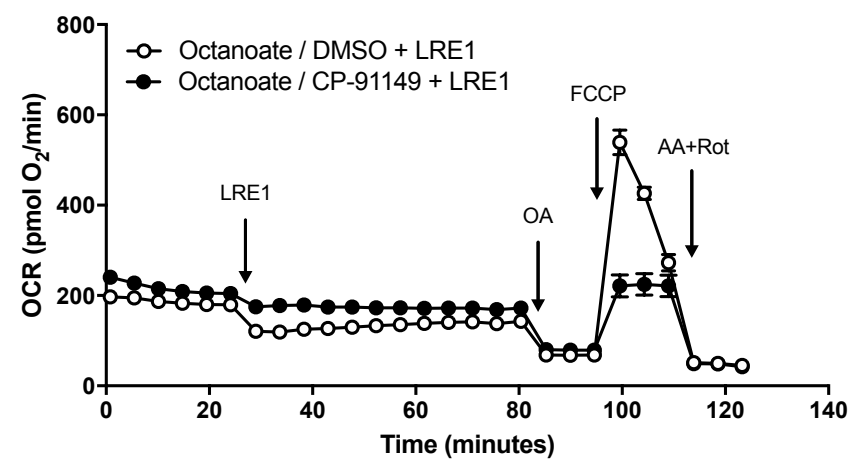

G

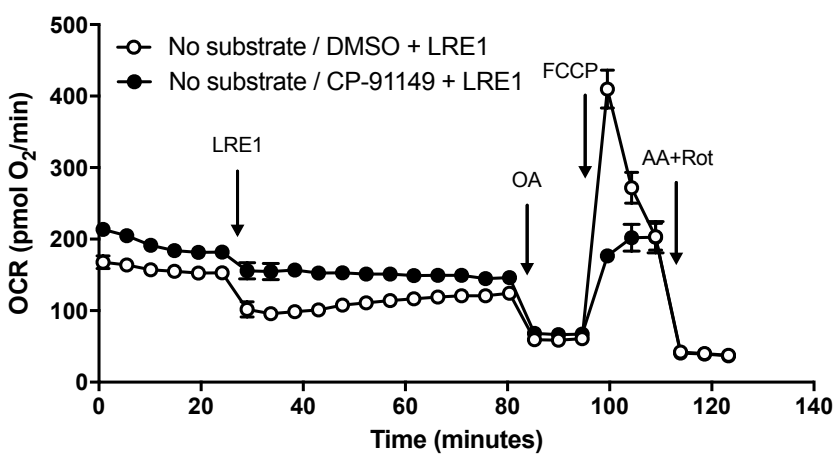

E

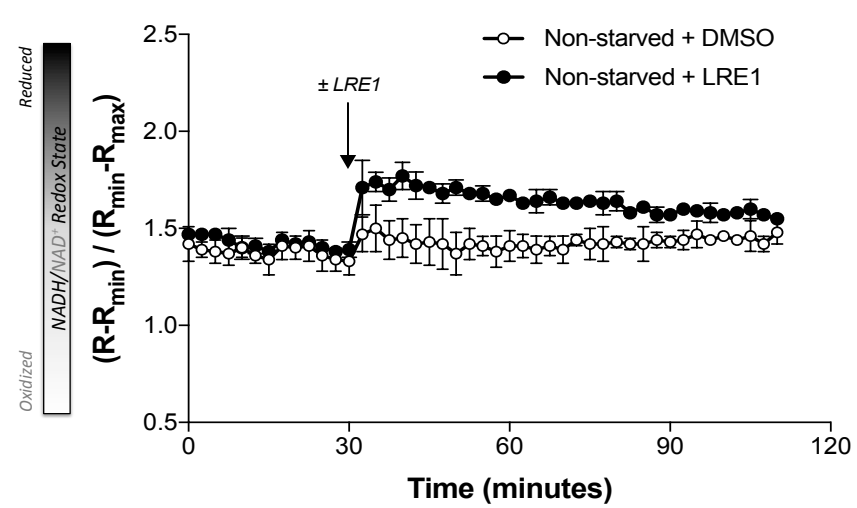

H

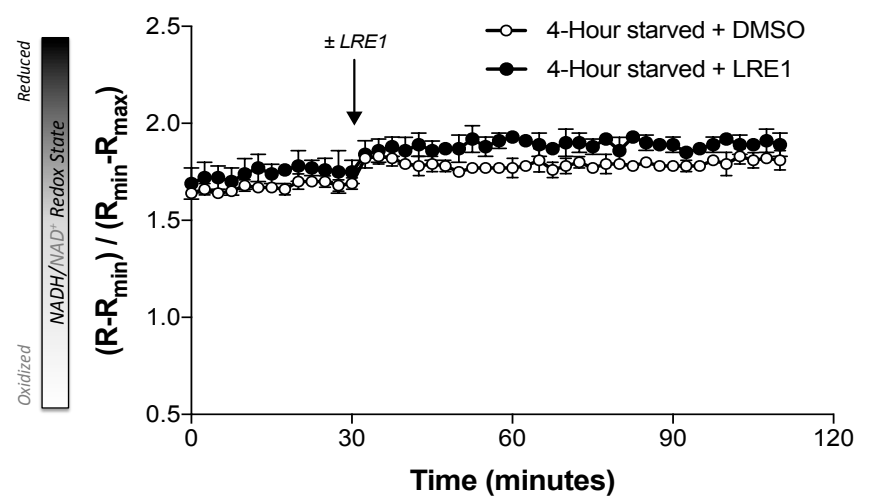


I

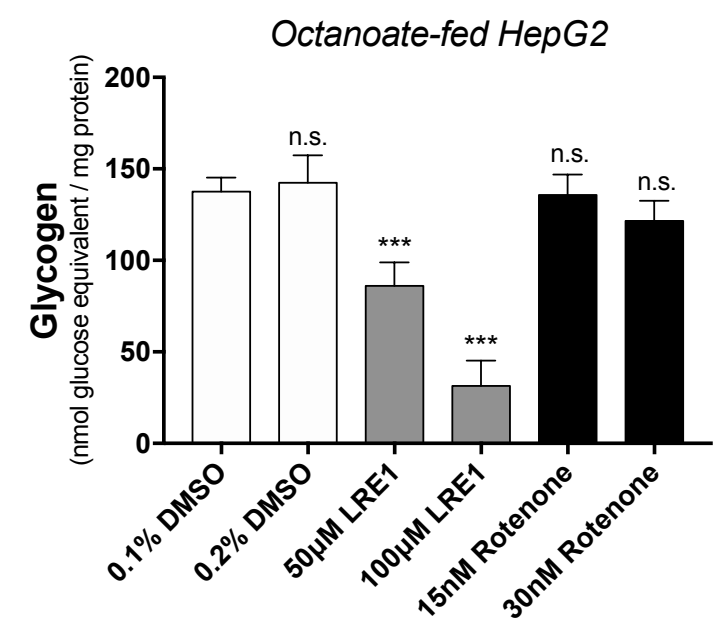

\section{J}

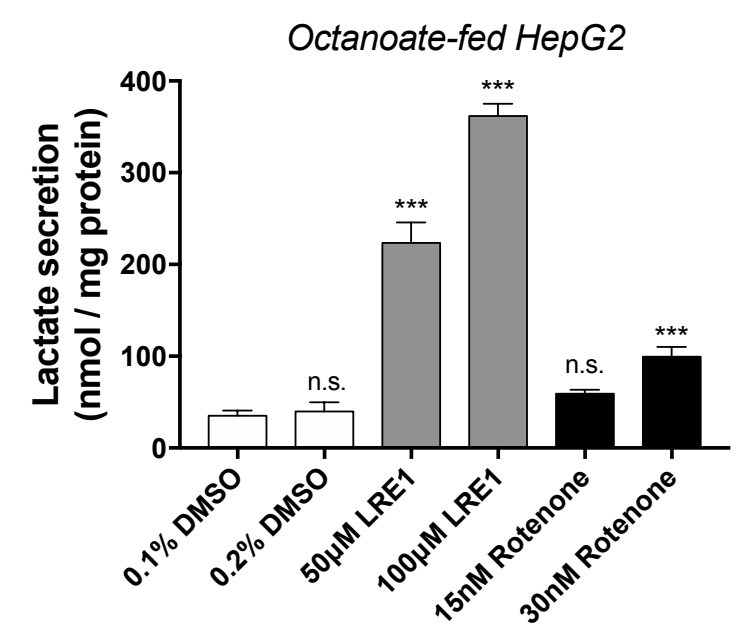

K

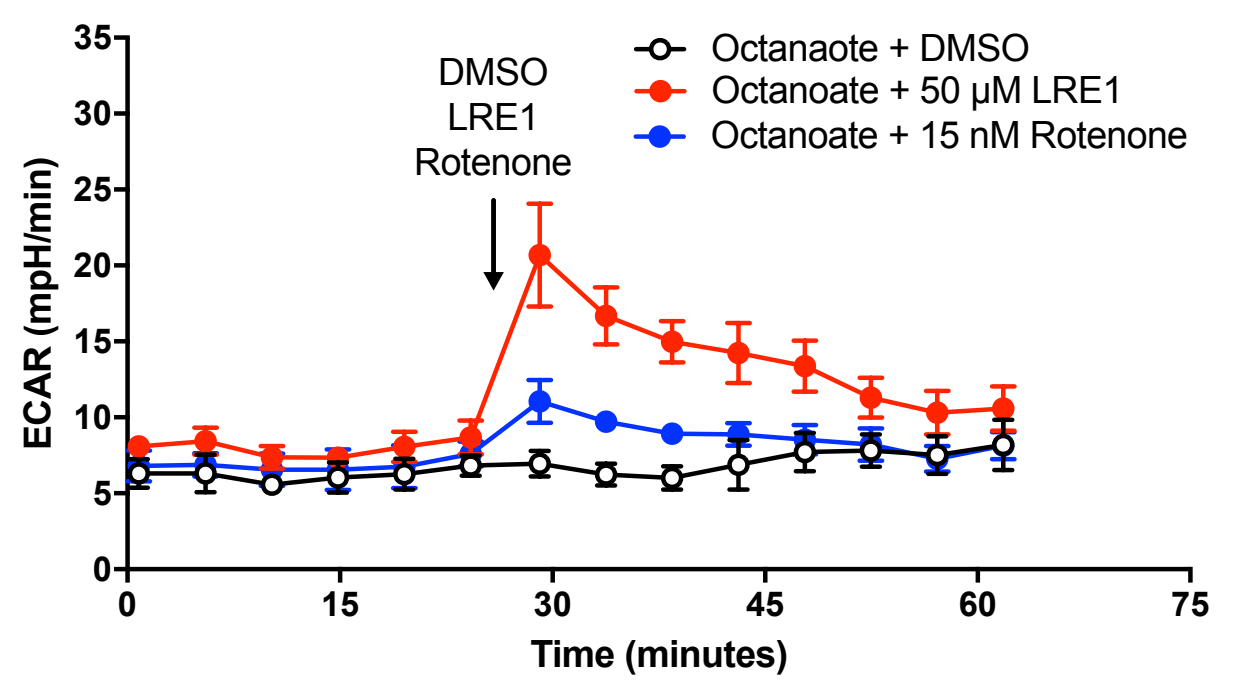




\section{Figure 5}

A

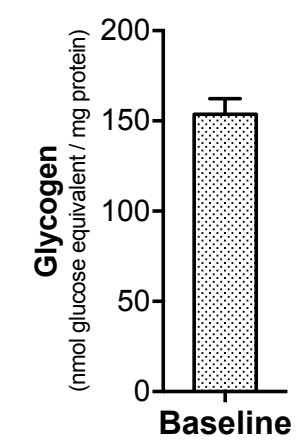

C

HepG2, 0 mM glucose
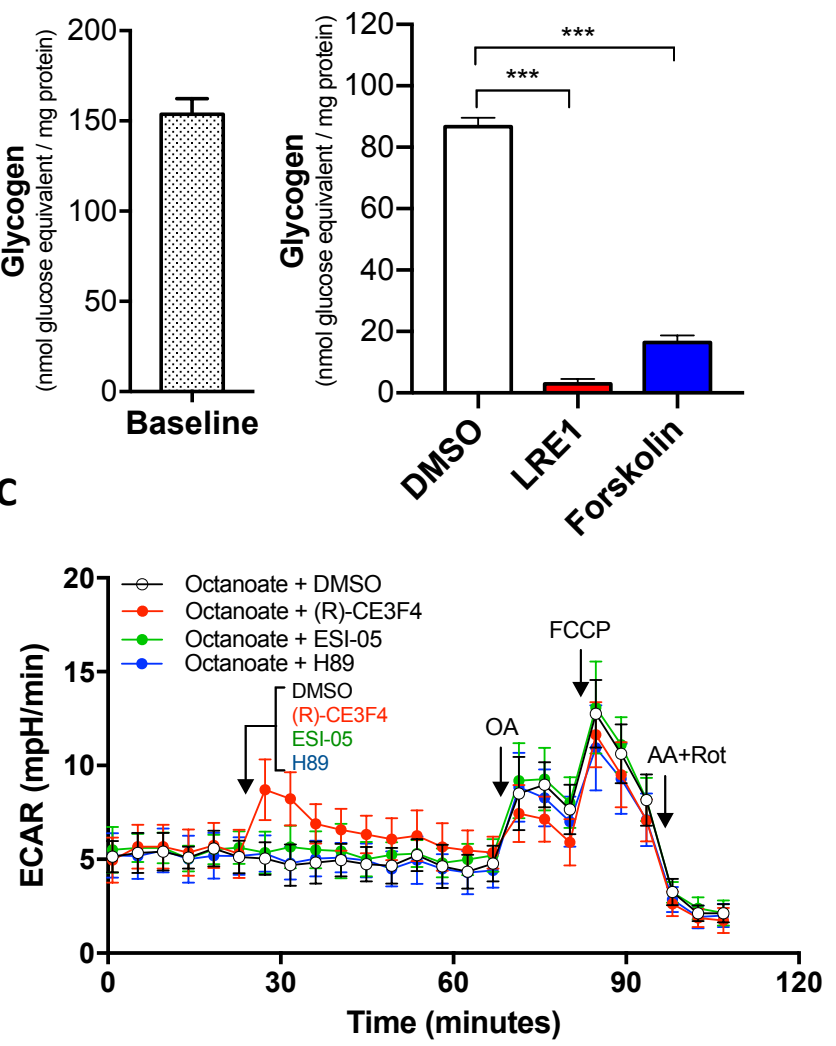

E

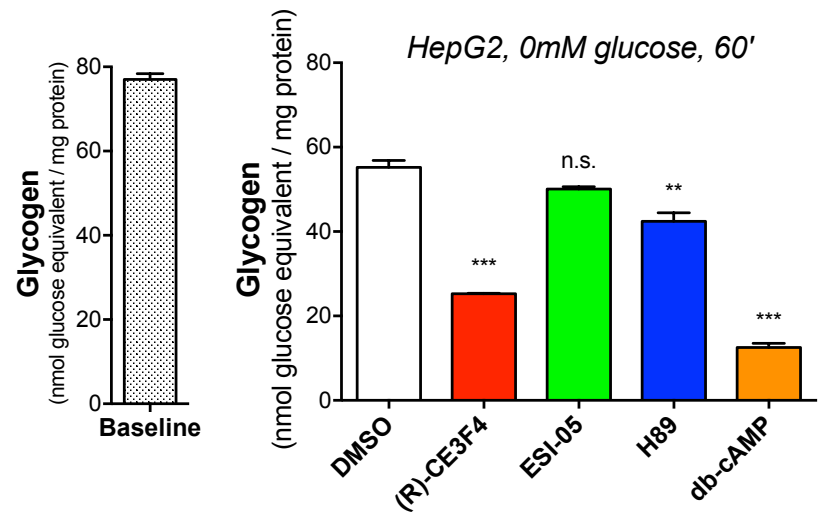

G

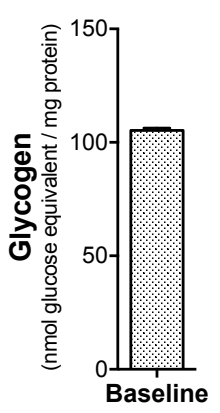

B
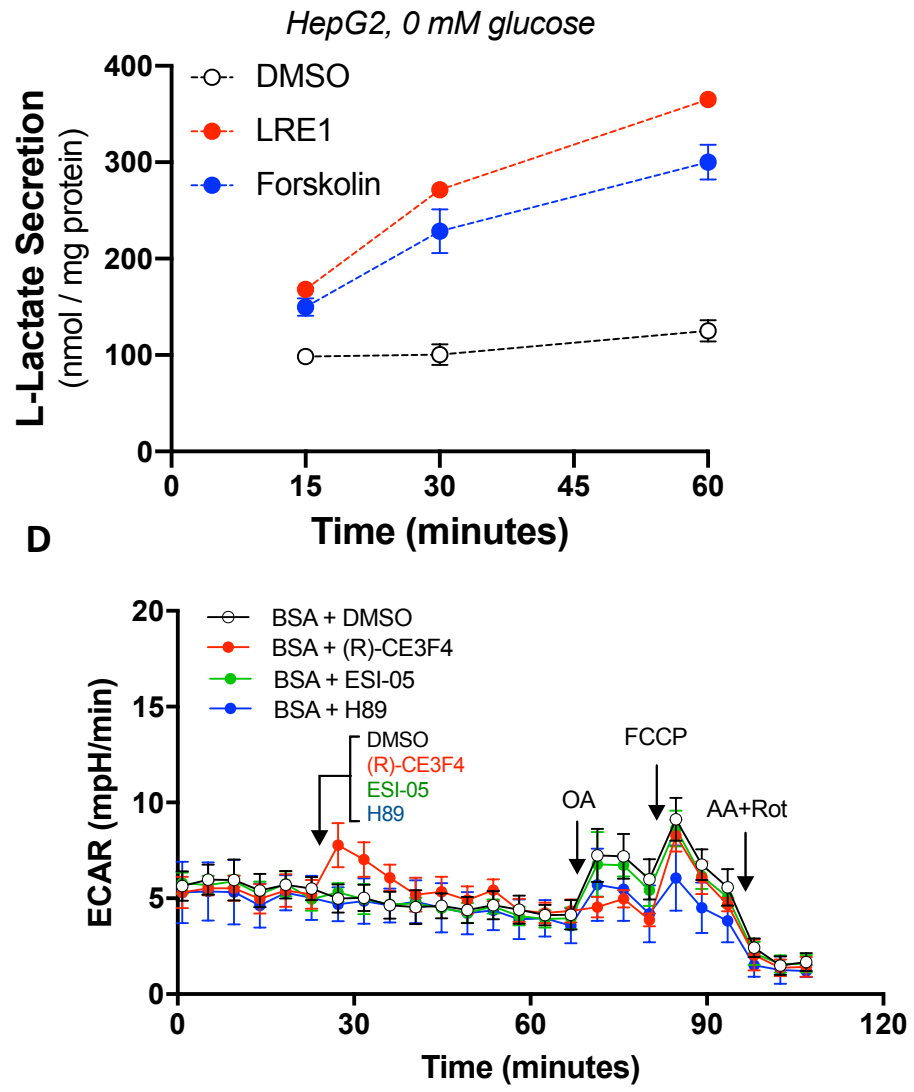

F
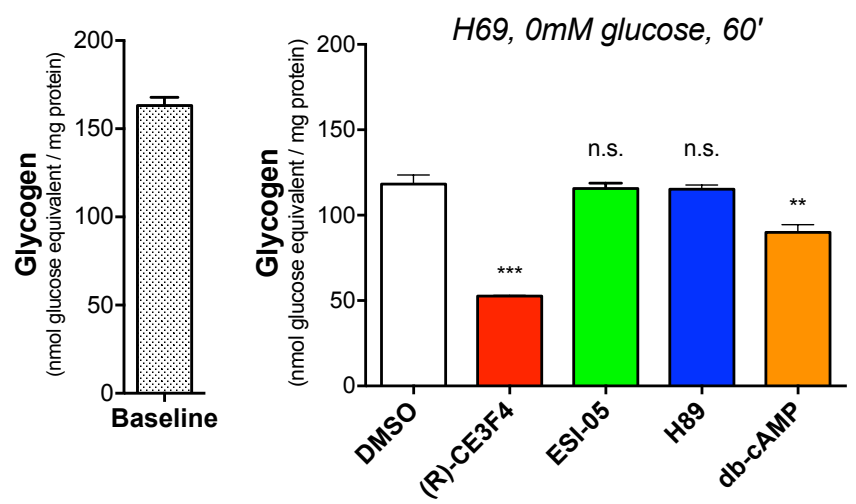

H
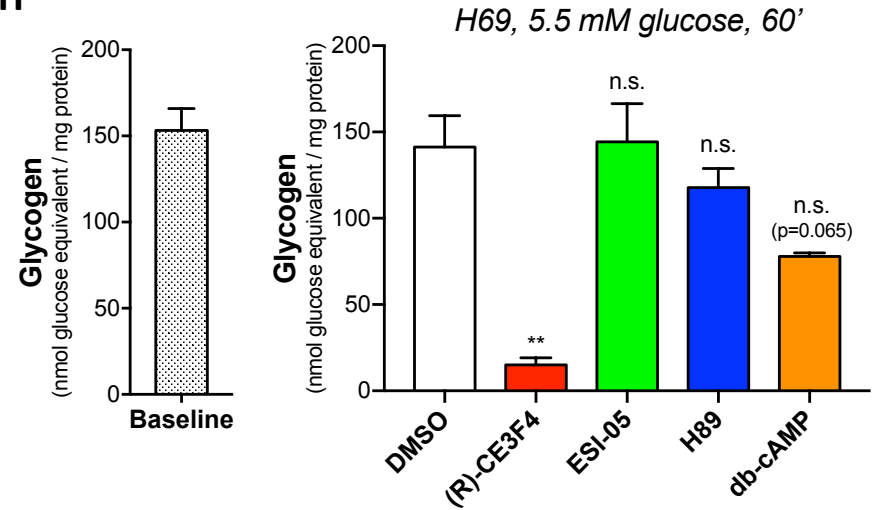


\section{Figure 5 (Continued)}

I

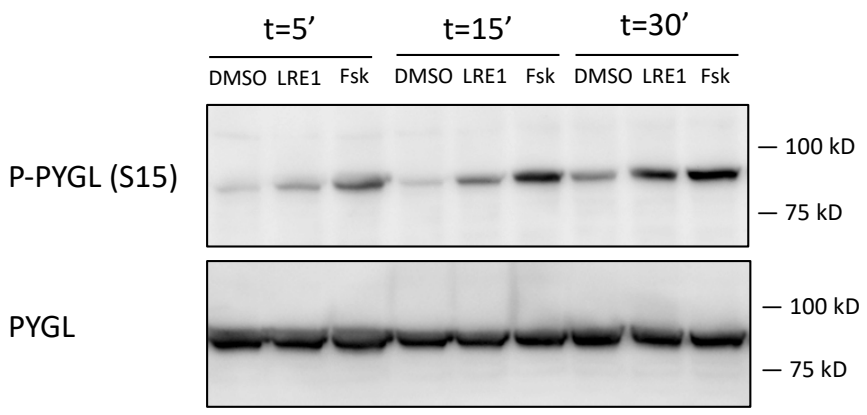

J

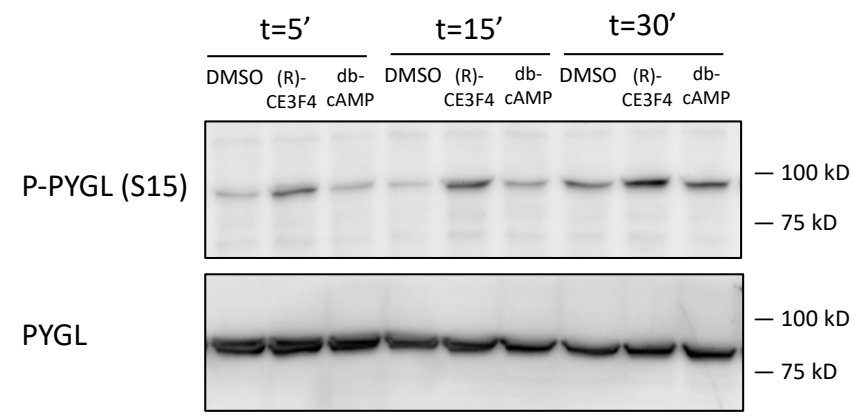


Figure 6

Glucose

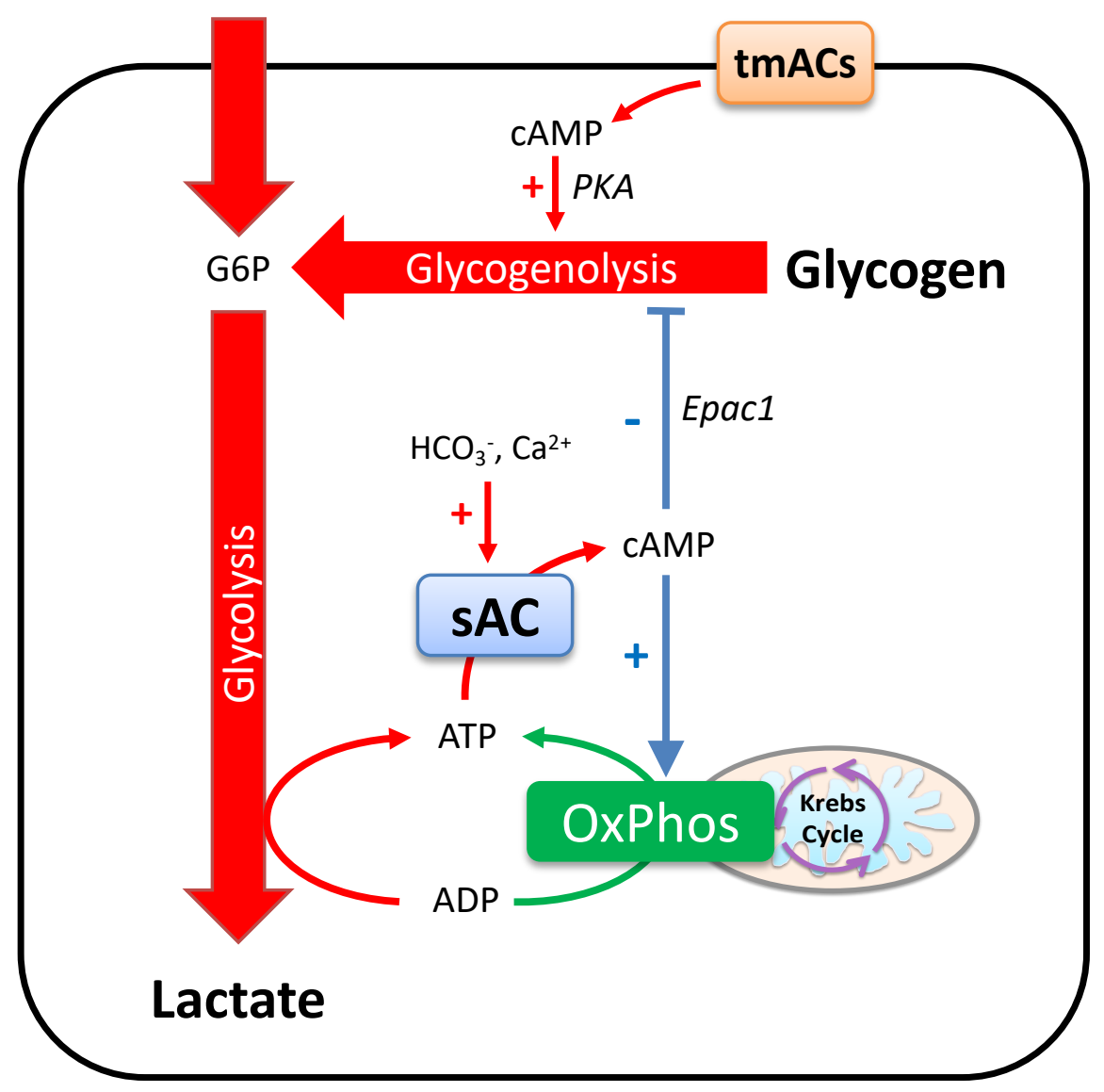


Figure S1

A

H69

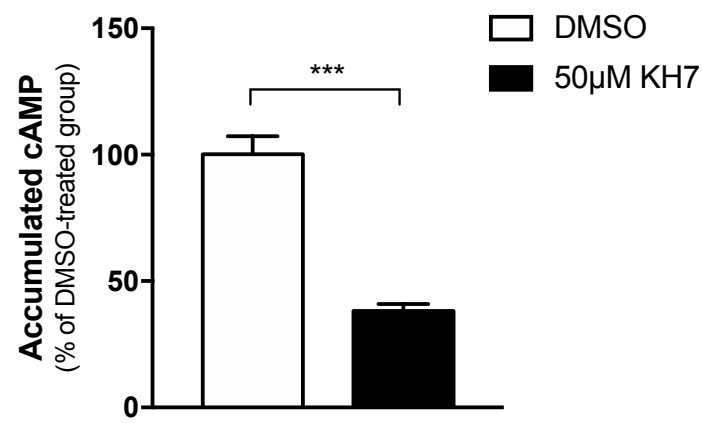

C
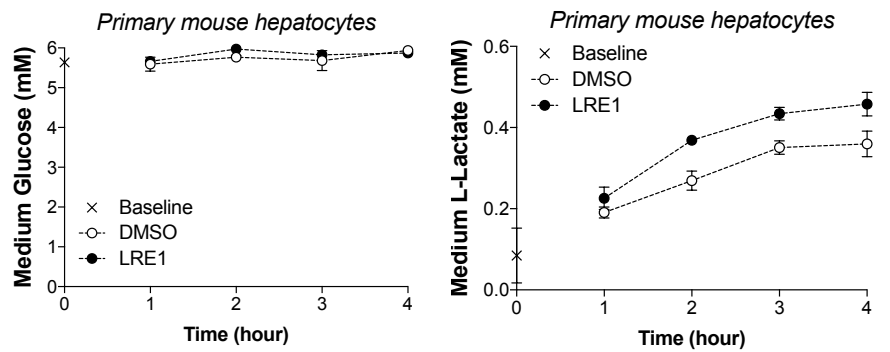

D

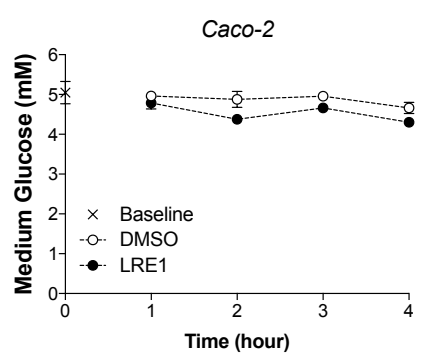

E

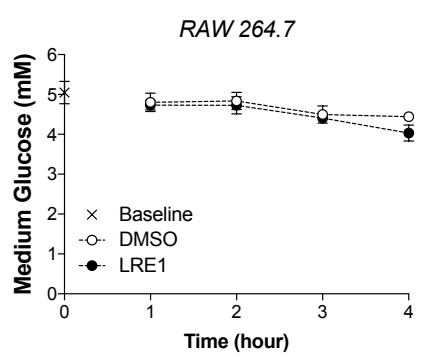

F
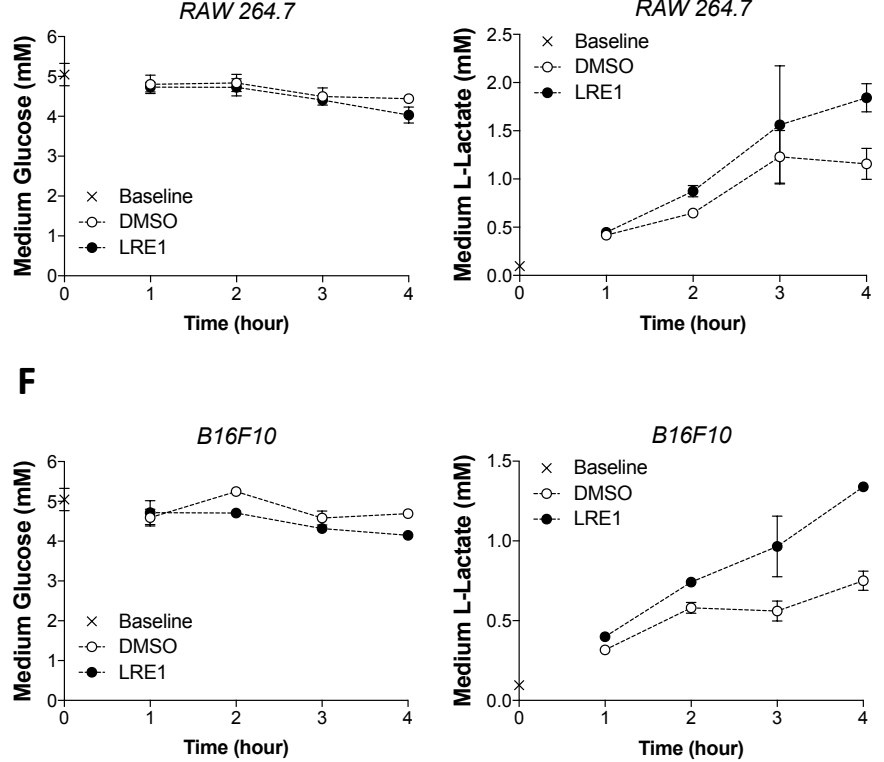

B

H69

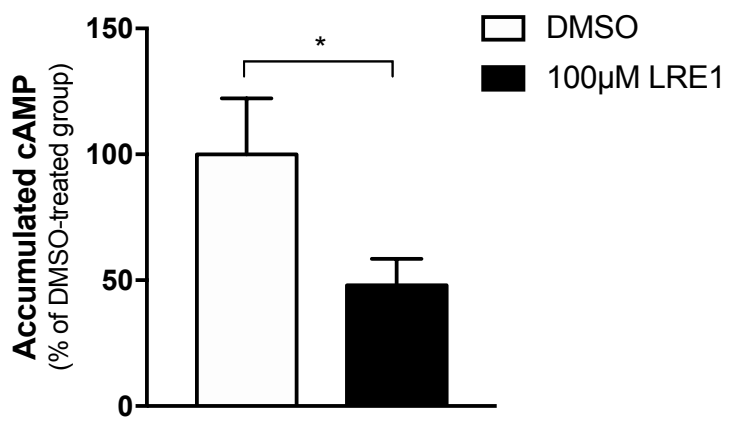

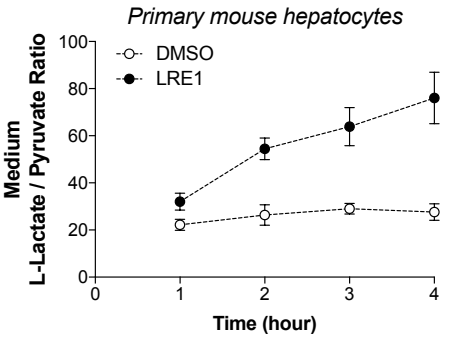

Caco-2

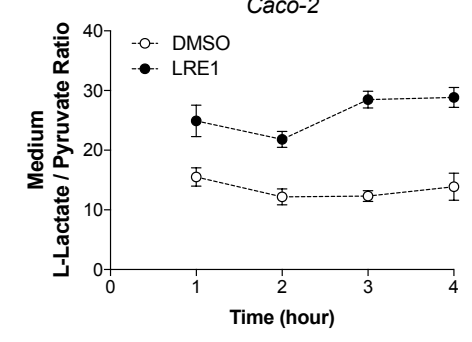

RAW 264.7
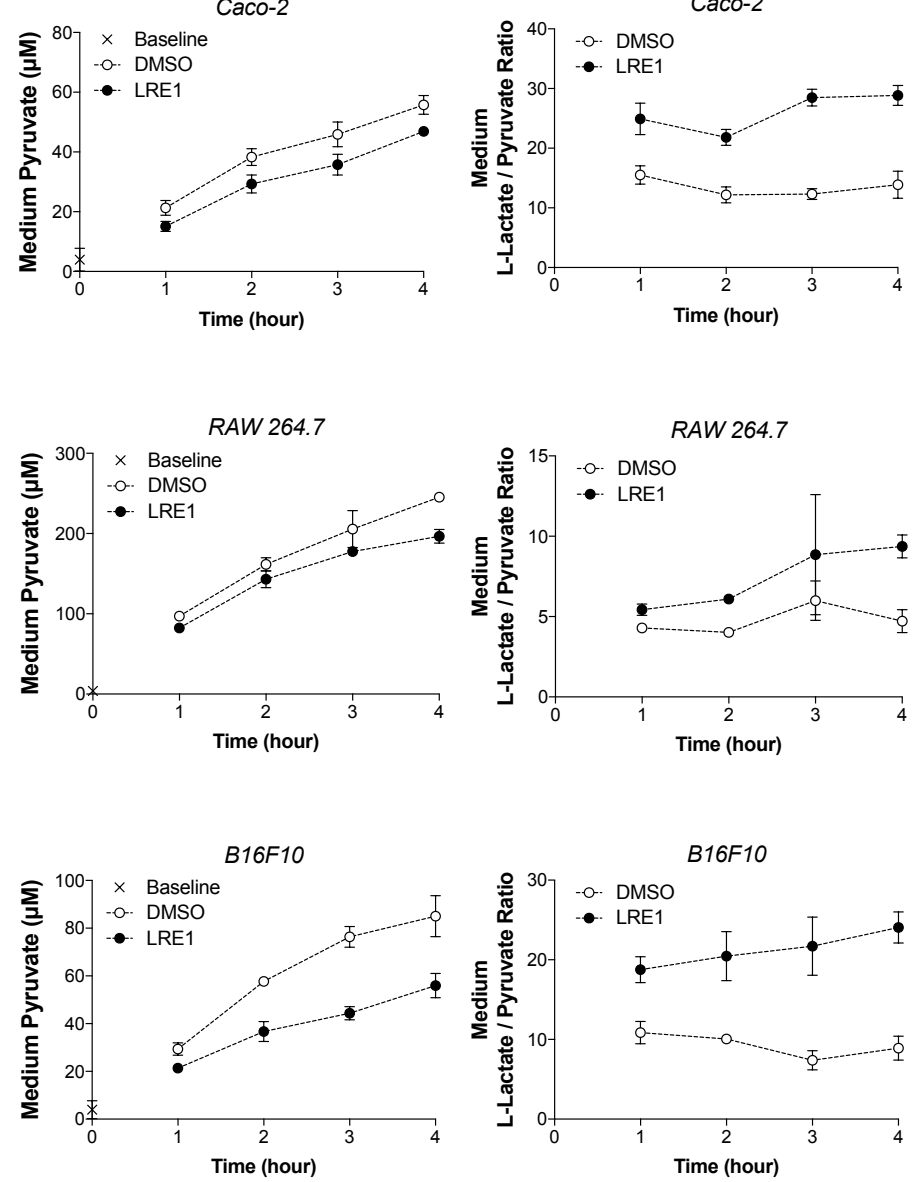
Figure S2

A

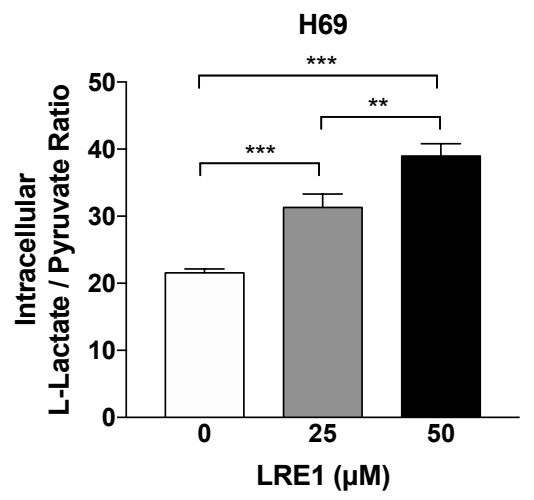

C

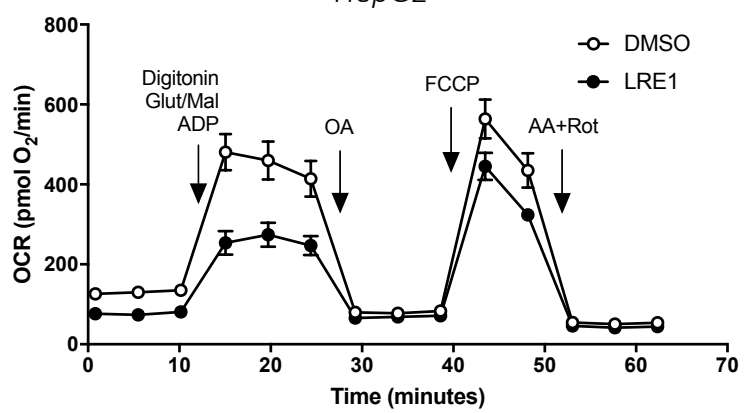

$\mathbf{F}$

HepG2, 5.5 mM glucose

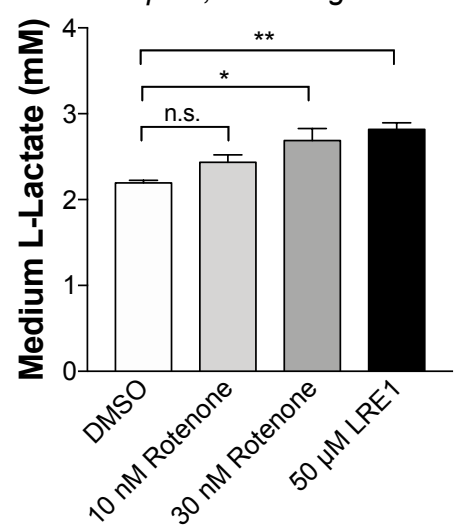

B

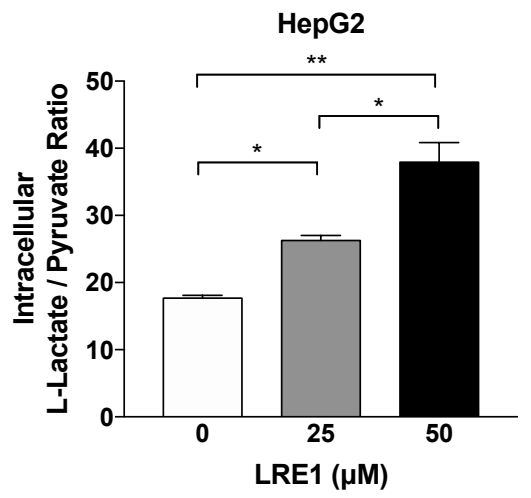

D

E
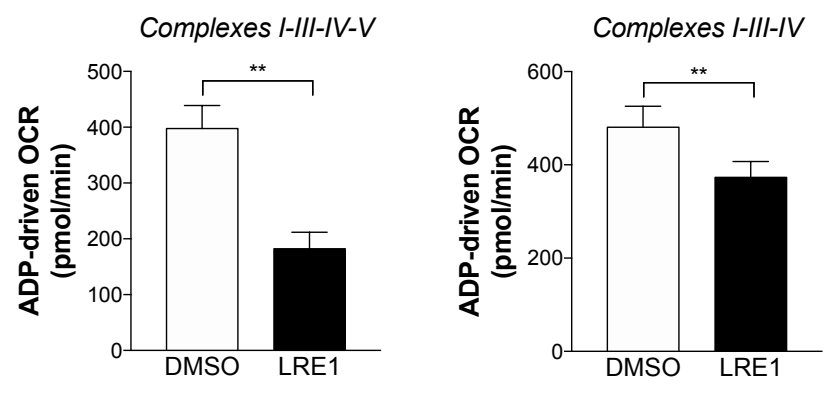

H
HepG2, 5.5 mM glucose

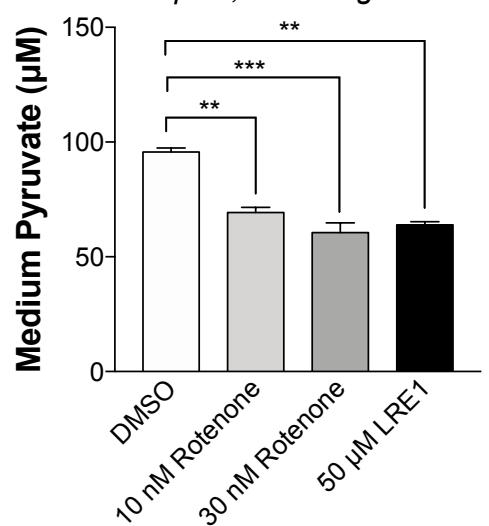

HepG2, 5.5 mM glucose

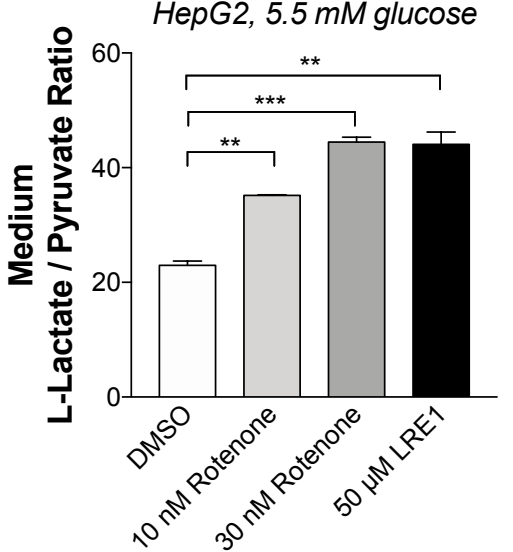


A

H69

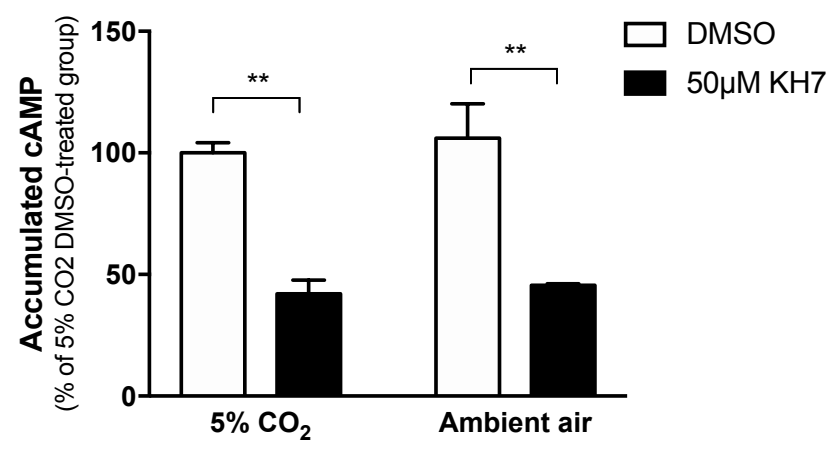

B

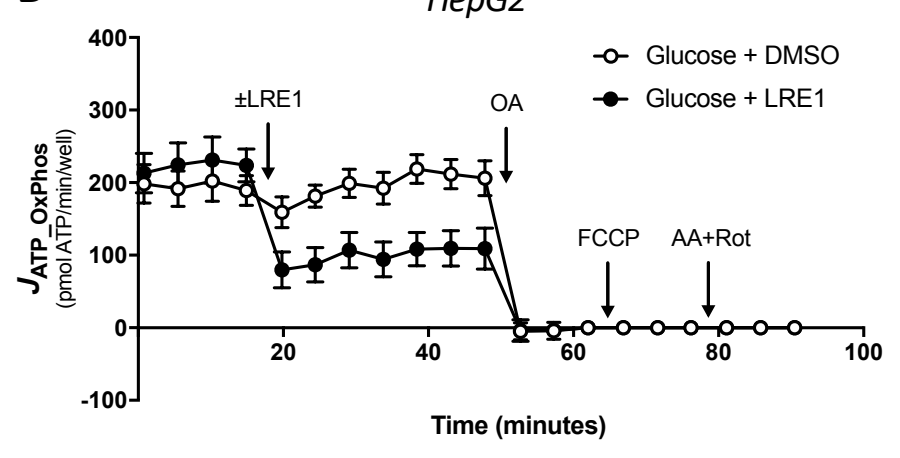

D

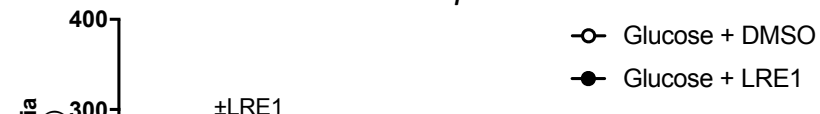

C

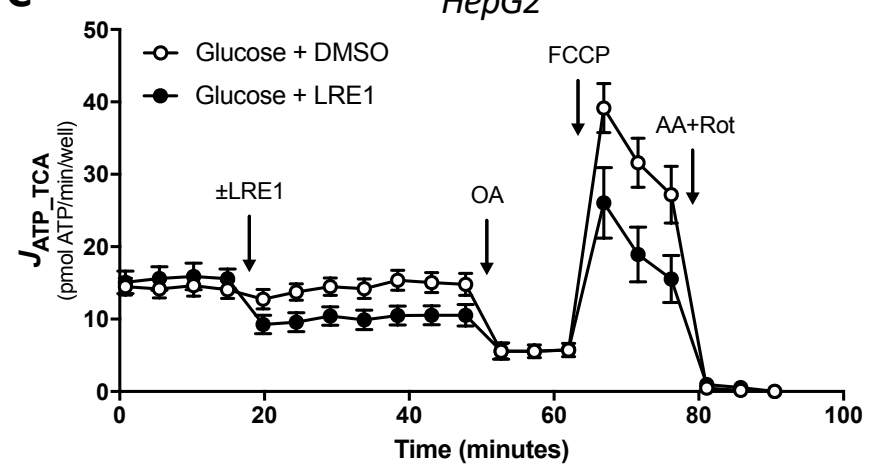

E

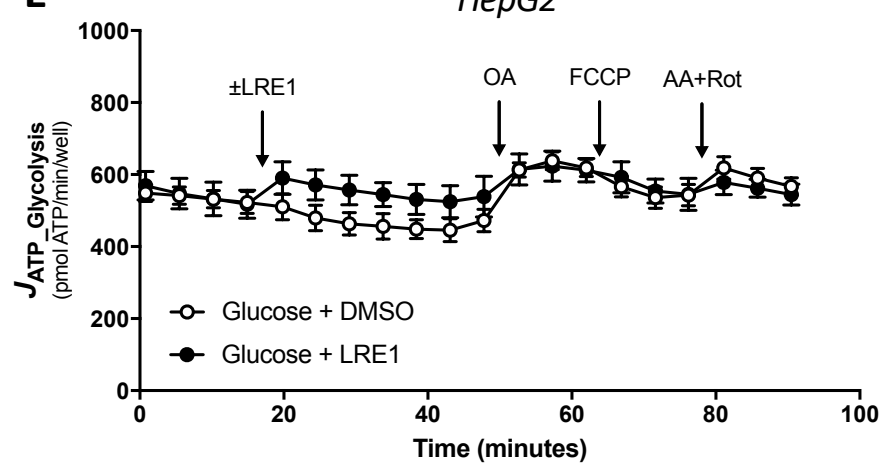

G
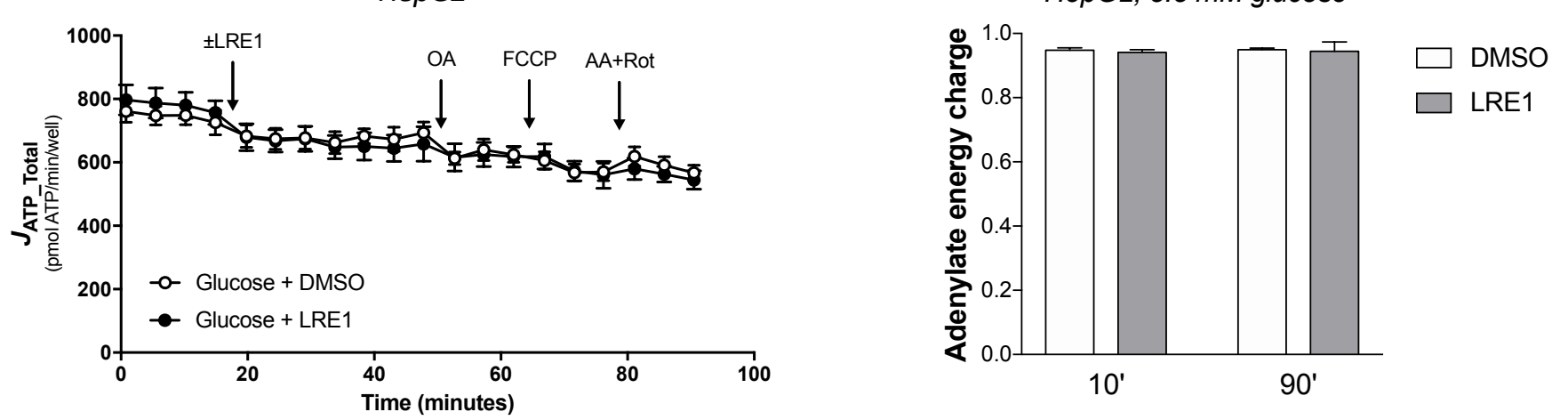
H

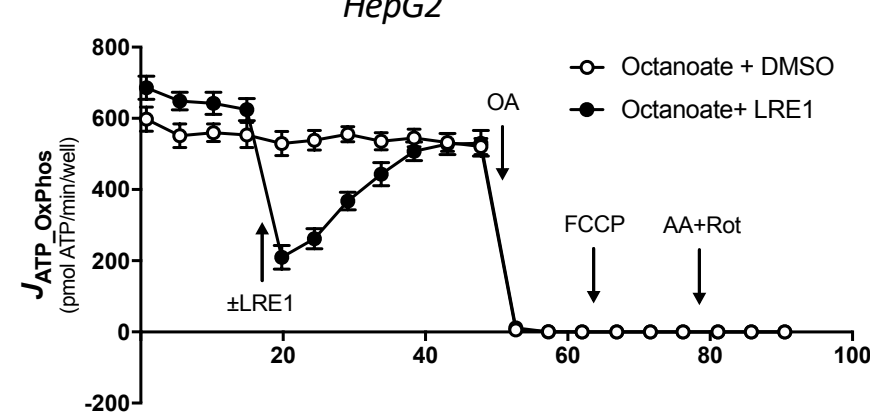

Time (minutes)

J

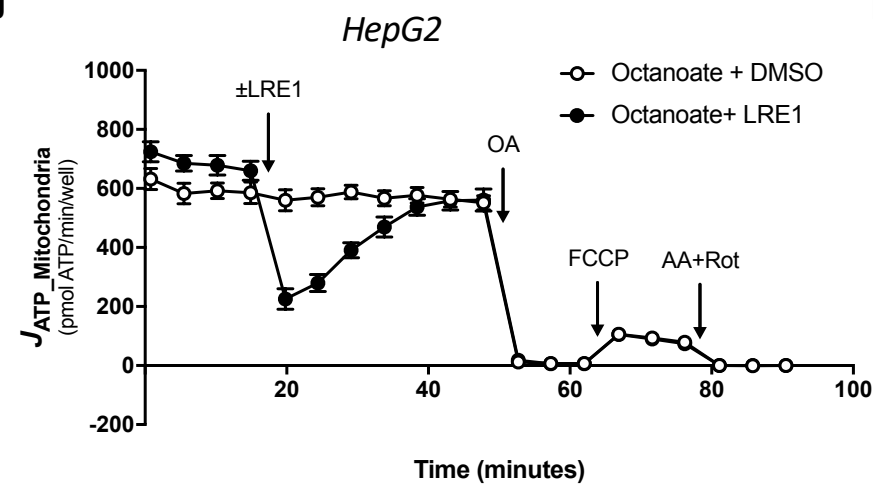

$\mathbf{L}$

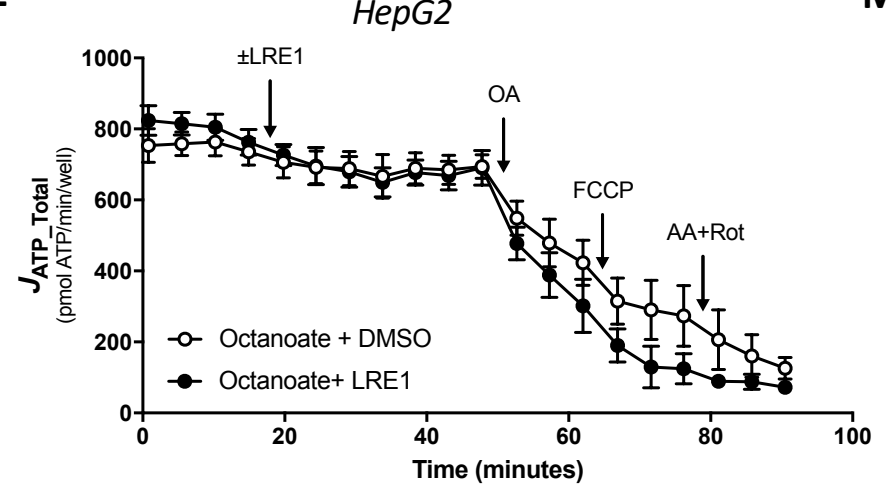

I

HepG2

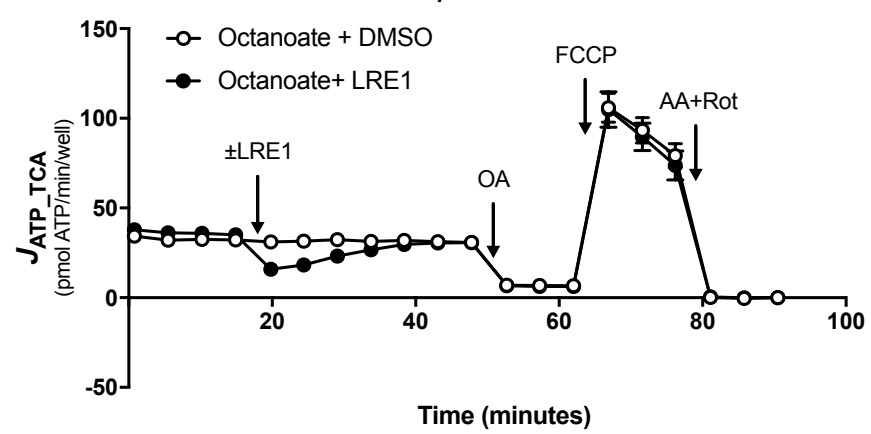

K

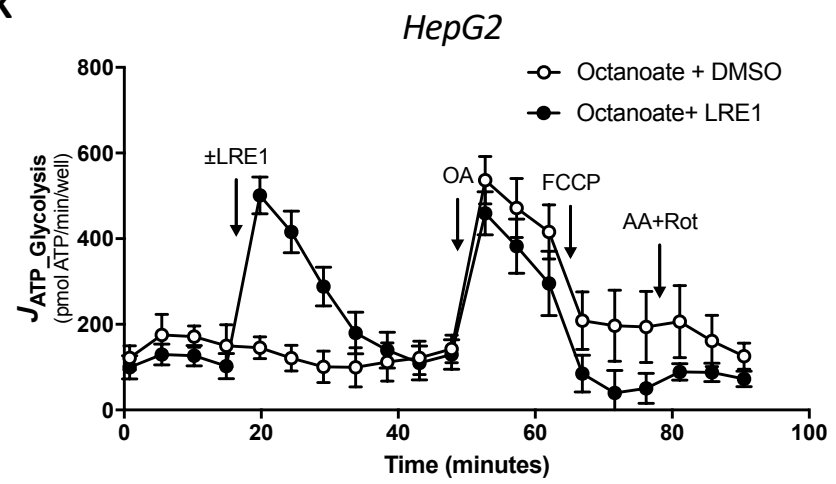

M

HepG2, 5.5 mM glucose

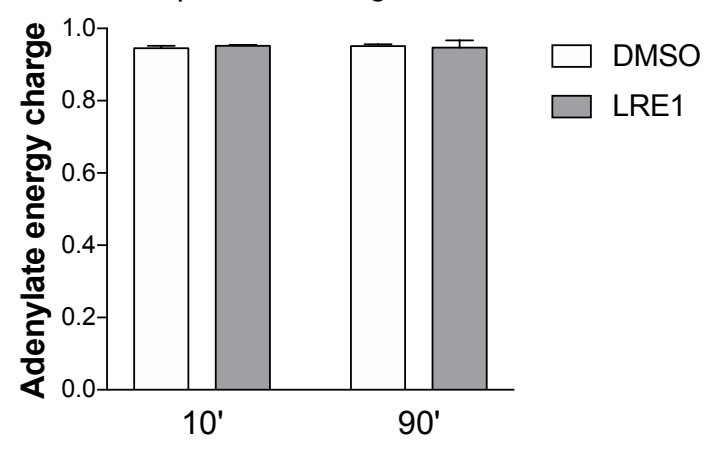


A

HepG2
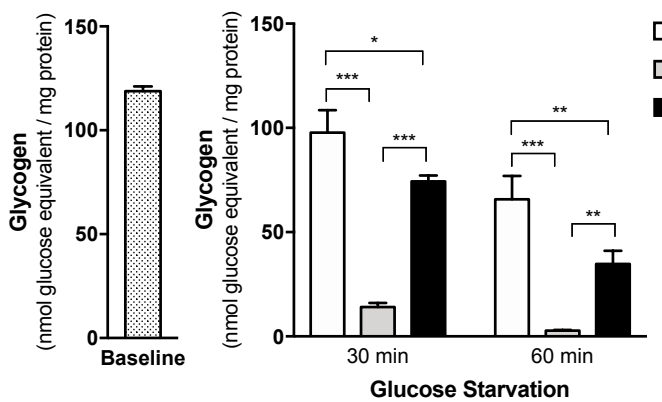

C

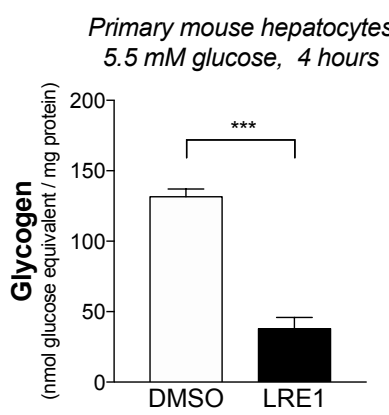

E

HepG2

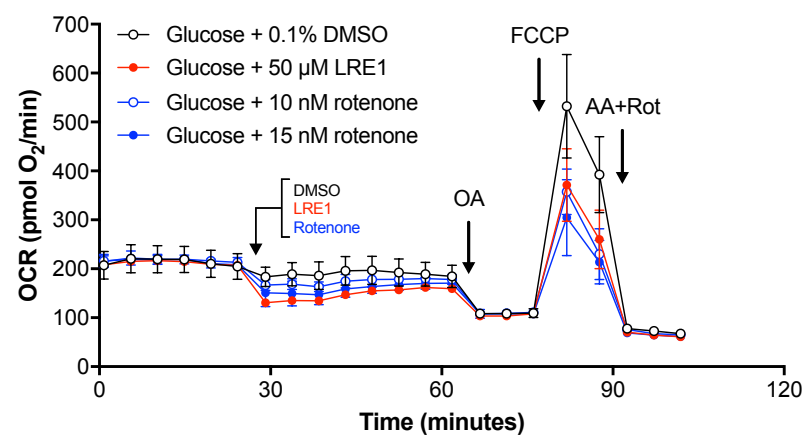

B

Primary mouse hepatocytes
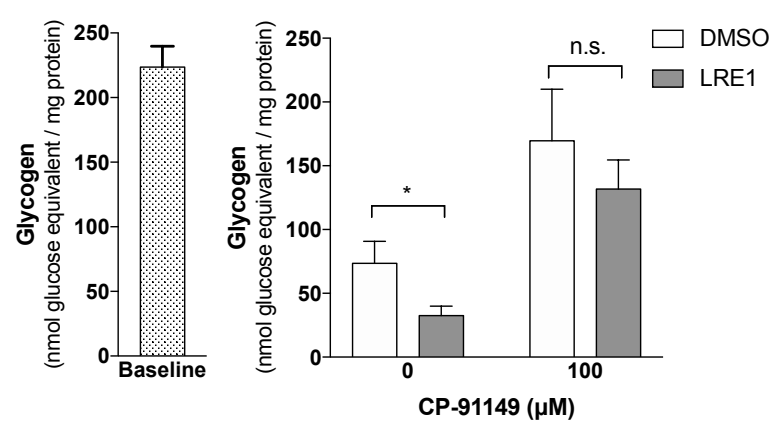

1.5hrs Starvation

D
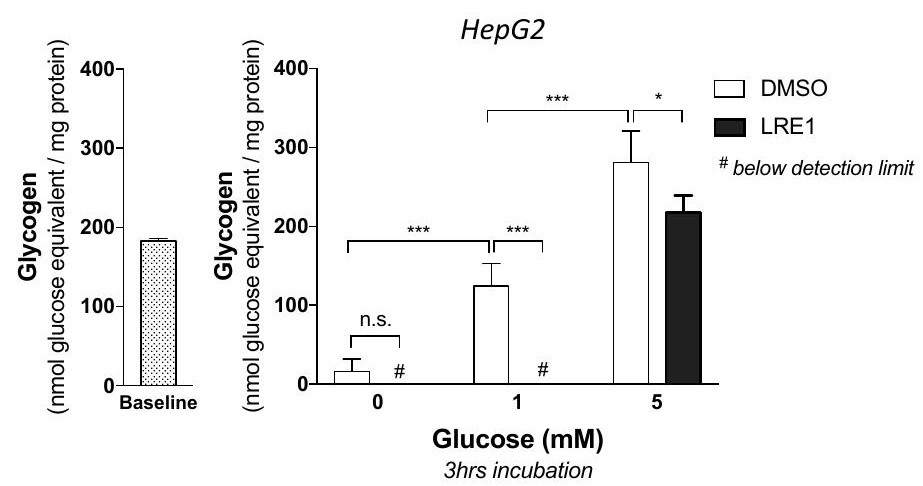

F

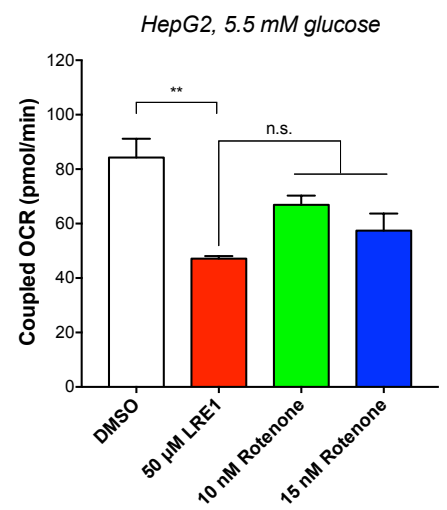

G

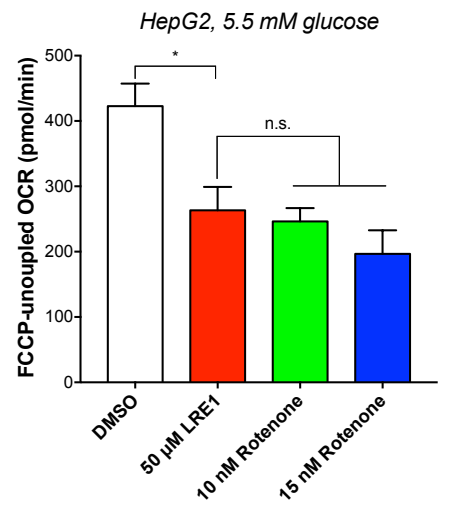


A

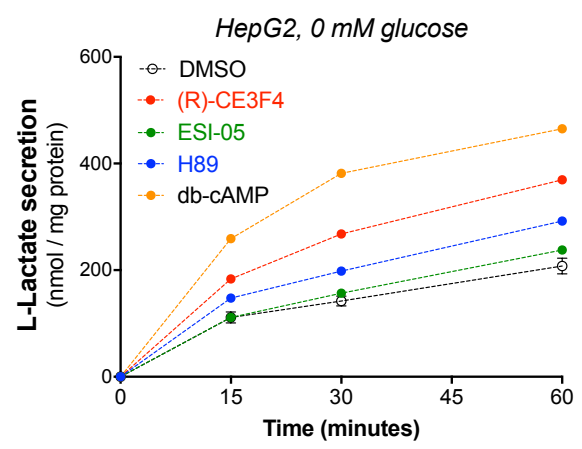

D

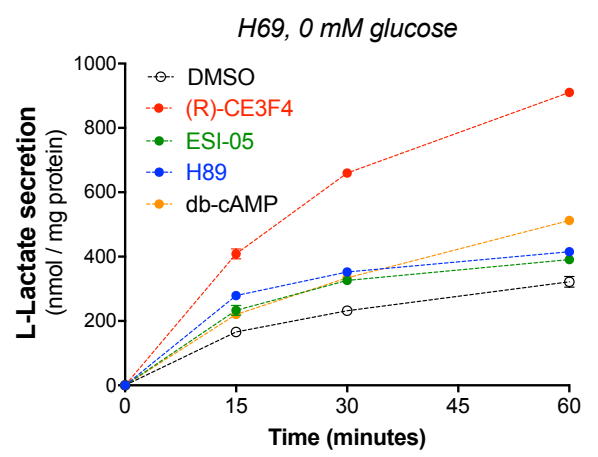

G

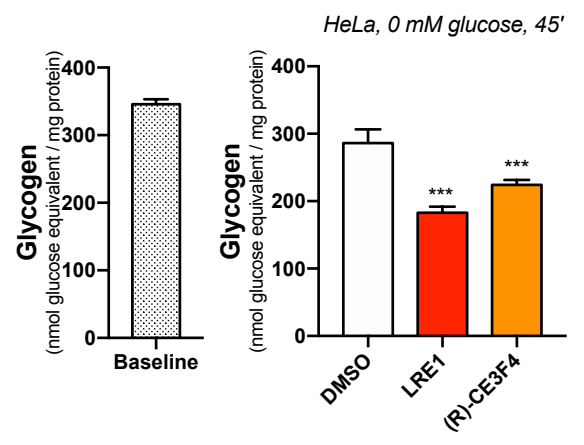

B

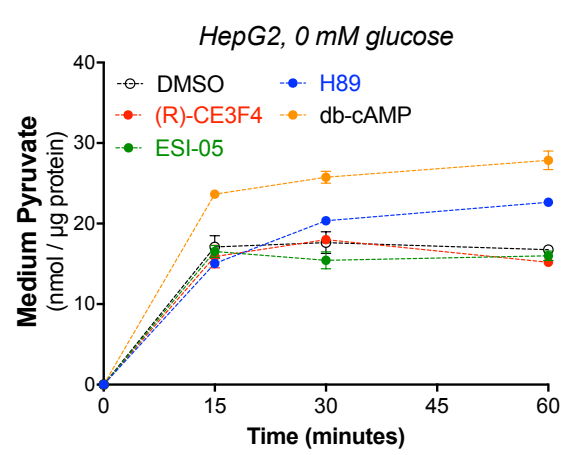

E

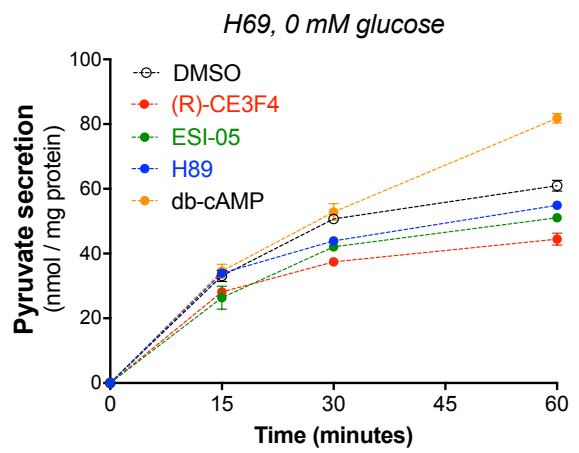

H

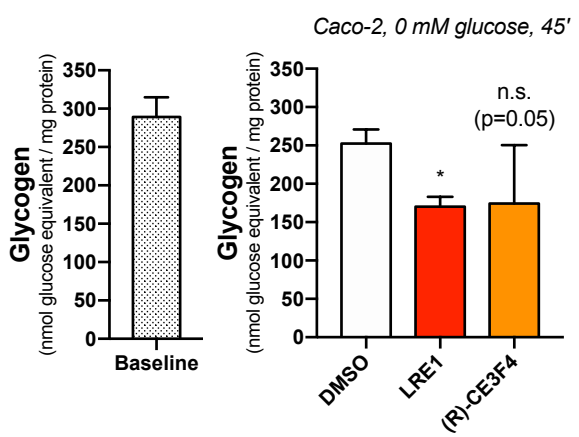

C

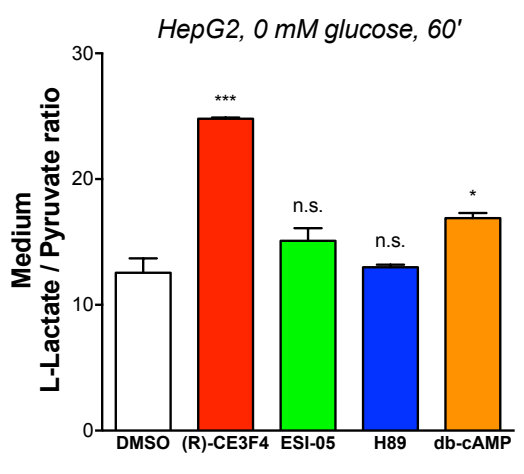

F

H69, o mM glucose, 60'

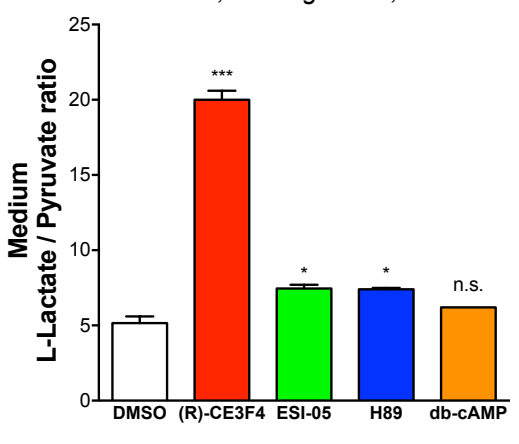

I

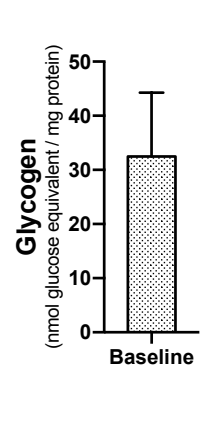

HEK293T, 0 mM glucose, 45'

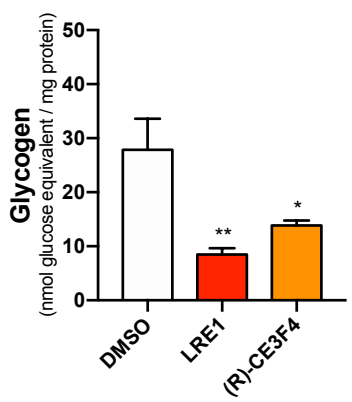

